\title{
Aerodynamic design of a tilting wind tunnel for the study of a glider in ground effect
}

Andres Felipe Velasquez E.

West Virginia University

Follow this and additional works at: https://researchrepository.wvu.edu/etd

\section{Recommended Citation}

Velasquez E., Andres Felipe, "Aerodynamic design of a tilting wind tunnel for the study of a glider in ground effect" (2009). Graduate Theses, Dissertations, and Problem Reports. 2087.

https://researchrepository.wvu.edu/etd/2087

This Thesis is protected by copyright and/or related rights. It has been brought to you by the The Research Repository @ WVU with permission from the rights-holder(s). You are free to use this Thesis in any way that is permitted by the copyright and related rights legislation that applies to your use. For other uses you must obtain permission from the rights-holder(s) directly, unless additional rights are indicated by a Creative Commons license in the record and/ or on the work itself. This Thesis has been accepted for inclusion in WVU Graduate Theses, Dissertations, and Problem Reports collection by an authorized administrator of The Research Repository @ WVU. For more information, please contact researchrepository@mail.wvu.edu. 


\section{AERODYNAMIC DESIGN OF A TILTING WIND TUNNEL FOR THE STUDY OF A GLIDER IN GROUND EFFECT}

Andrés Felipe Velásquez E.

Thesis submitted to the

College of Engineering and Mineral Resources

at West Virginia University

in partial fulfillment of the requirements

for the degree of

Master of Science

in

Aerospace Engineering

James E. Smith, Ph.D., Committee Chairperson

Gerald M. Angle II, Ph.D.

Jacky C. Prucz, Ph.D.

Department of Mechanical and Aerospace Engineering

Morgantown, West Virginia

2008

Keywords: Tilting Wind Tunnel, Wind Tunnel Design,

Ground Effect Testing, Moving-Belt Ground Plane

Copyright 2008 Andrés Felipe Velásquez E. 


\title{
Abstract \\ AERODYNAMIC DESIGN OF A TILTING WIND TUNNEL FOR THE STUDY OF A GLIDER IN GROUND EFFECT
}

\author{
Andrés Felipe Velásquez E.
}

A small, low-speed, open return, tilting wind tunnel has been designed. This wind tunnel is intended to simulate the behavior of an unpowered recreational glider that flies down slopes in ground effect at about $1 \mathrm{~m}$ above the surface. The required tilting angle is $50^{\circ}$. The wind tunnel has to be capable of holding free-flying models with a recommended scaling factor of 14.2 and with Froude number matching.

The designed wind tunnel uses a centrifugal fan to blow air into the test section through a wide angle diffuser, a settling chamber and a contraction. There are six screens and a honeycomb to improve flow uniformity. The test section is closed and rectangular $(384 \mathrm{~mm}$ by $576 \mathrm{~mm})$. The maximum obtainable test section velocity is approximately $35 \mathrm{~m} / \mathrm{s}$. A moving belt is used to simulate the ground. All the components were designed following methods recommended in literature and the contraction was designed using CFD simulations. 


\section{Acknowledgments}

I would like to express my gratitude to my advisor, Dr. Smith, for his support during this research.

I would like to thank the members of my committee, Dr. Jacky Prucz and Dr. Gerald Angle for their suggestions and their participation in this project; I also want to thank Dr. Jacky Prucz for his support and advice during my graduate program.

I would like to thank Jonathan Kweder for his help and information in the development of this project. 


\section{TABLE OF CONTENTS}

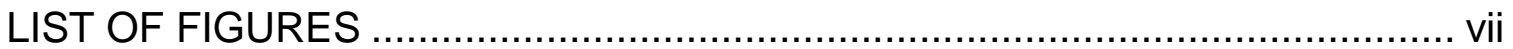

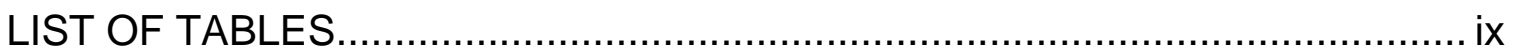

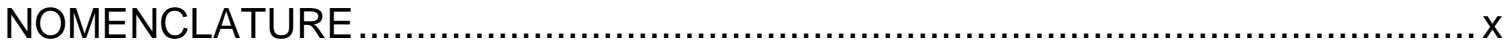

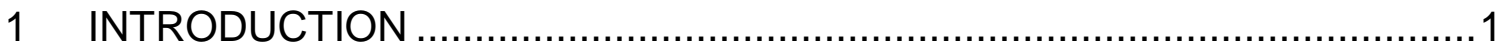

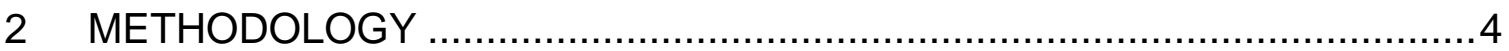

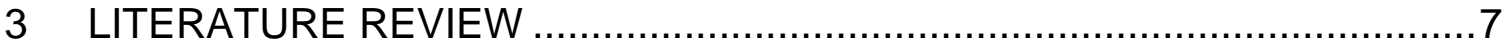

3.1 WIND TUNNEL DESCRIPTION AND STATE OF THE ART …............. 7

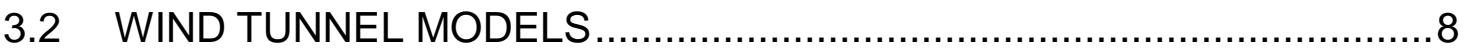

3.2.1 Mounted Wind Tunnel Models..................................................

3.2.2 Free Flying Models..............................................................

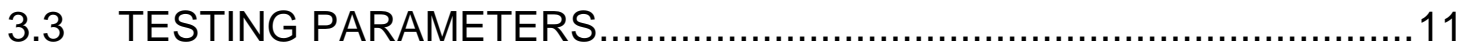

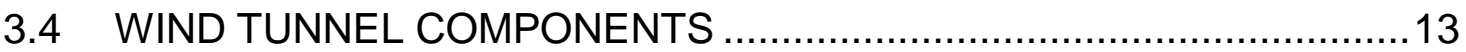

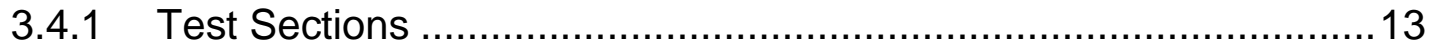

3.4.1.1 Cross-Sectional Shape of the Test Section.........................................13

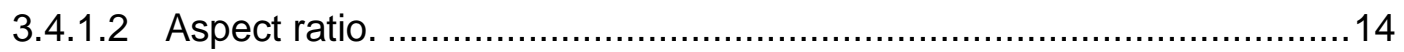

3.4.1.3 Open and Closed Test Sections. ..................................................15

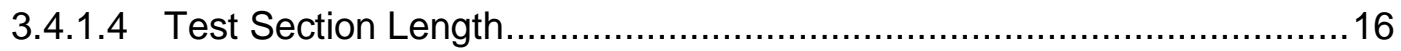

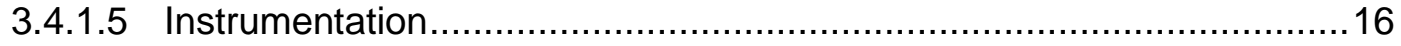

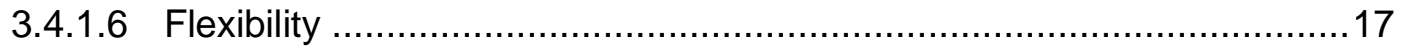

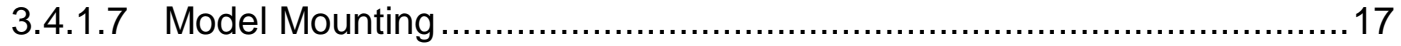

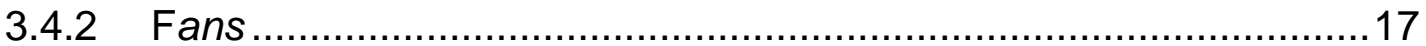

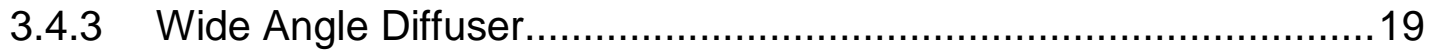

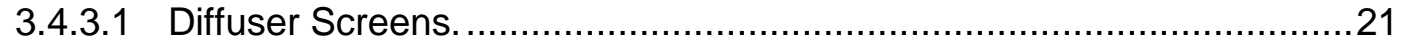

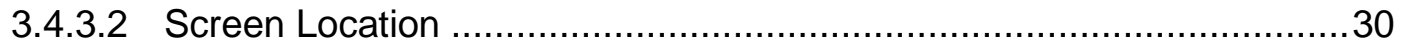

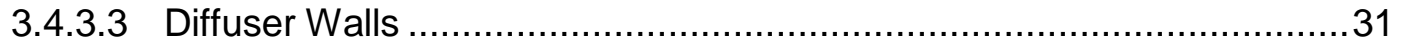

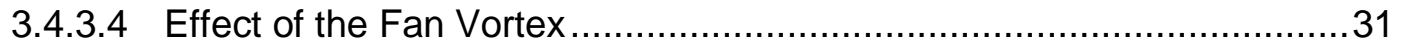

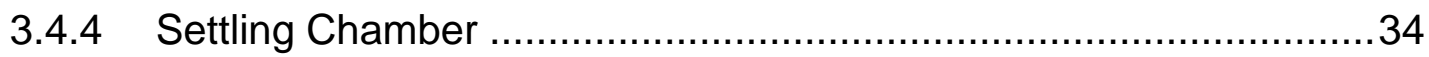

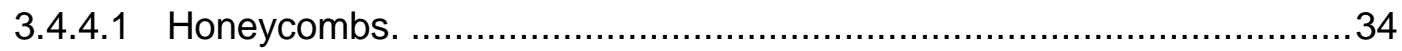

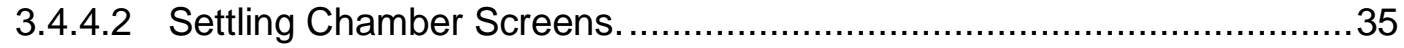

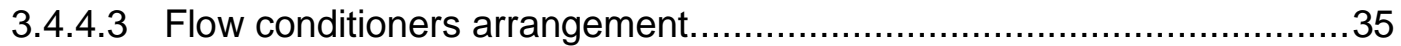




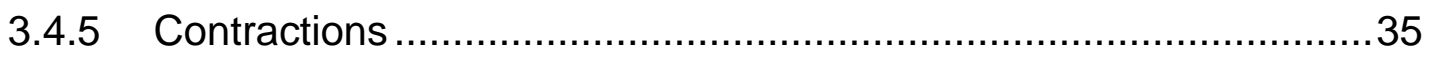

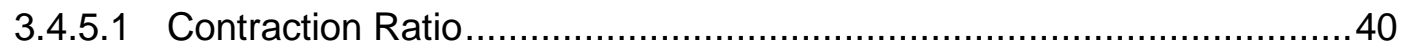

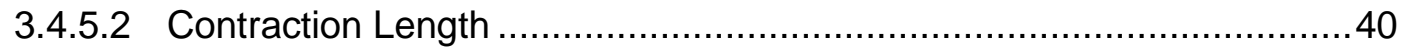

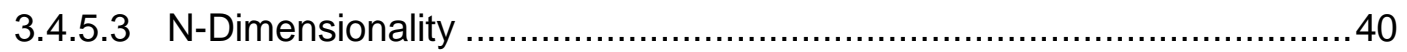

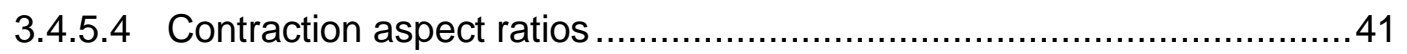

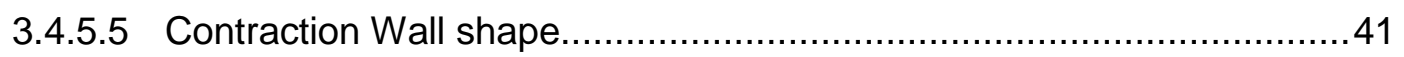

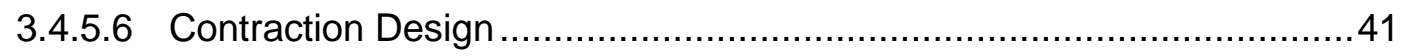

3.5 WIND TUNNEL CORRECTION FACTORS .....................................45

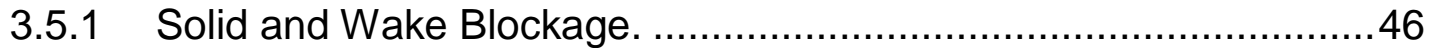

3.5.2 Alteration of the local angle of attack along the span ...................46

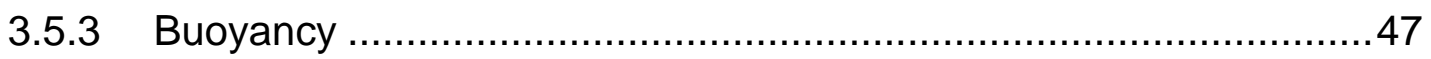

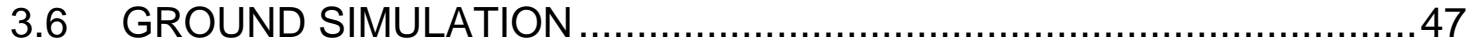

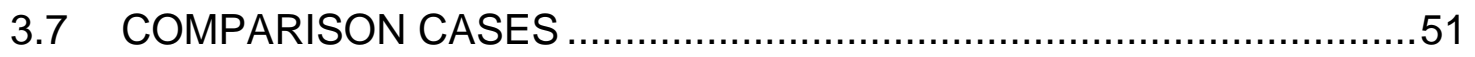

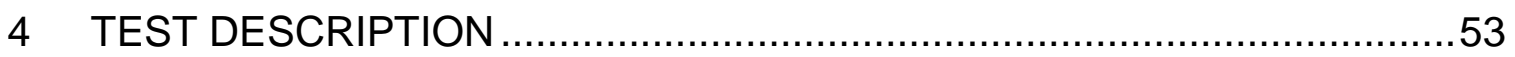

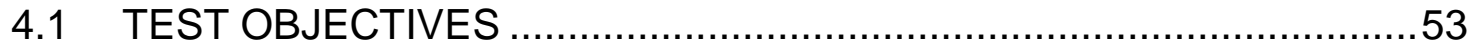

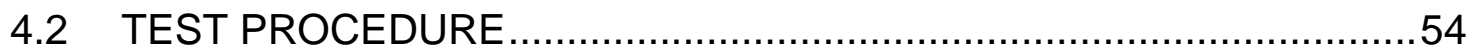

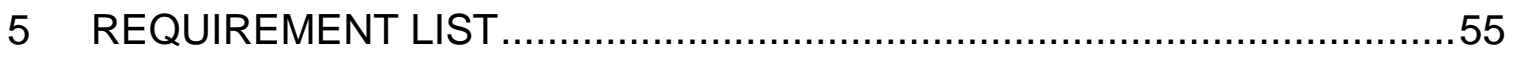

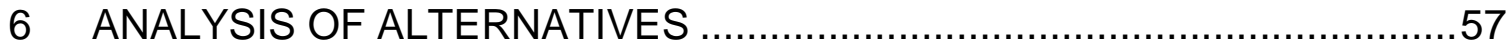

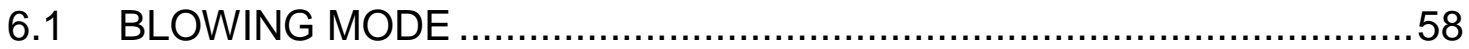

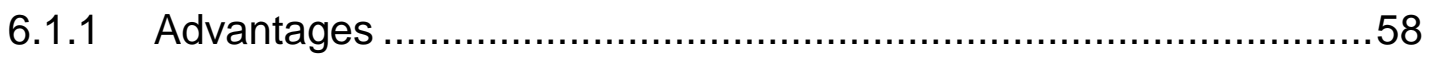

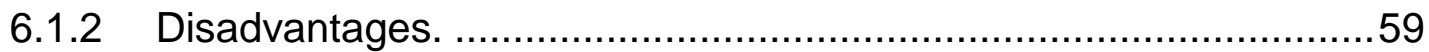

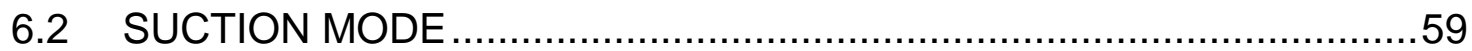

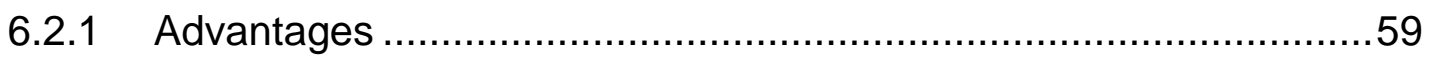

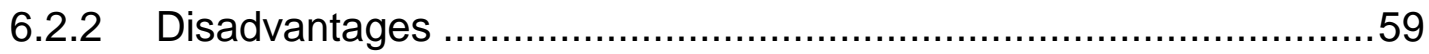

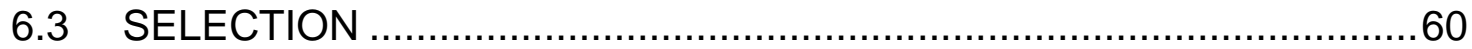

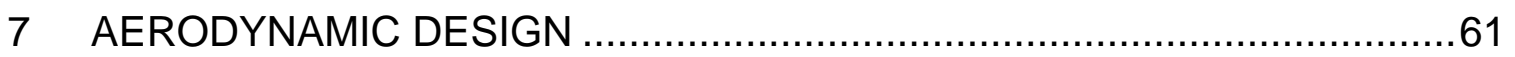

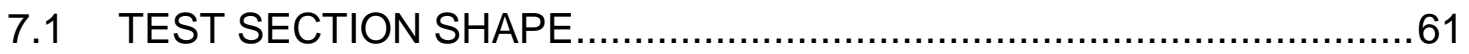

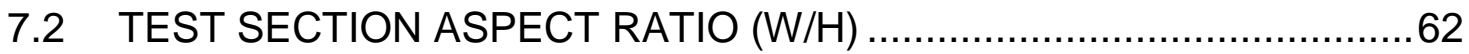

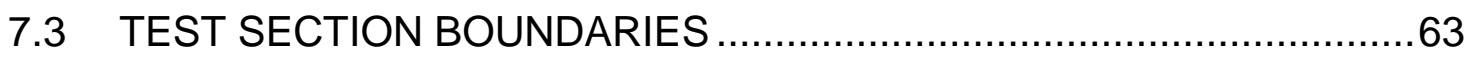

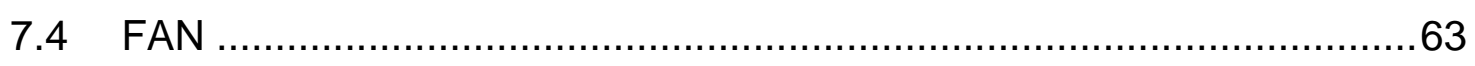

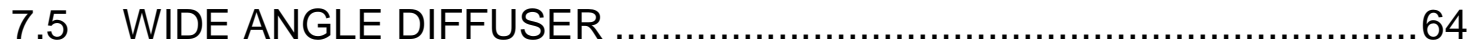




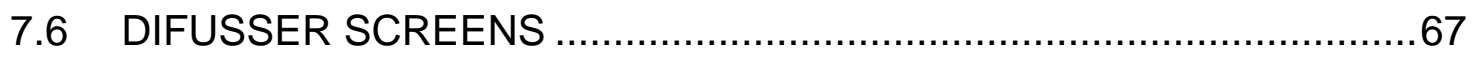

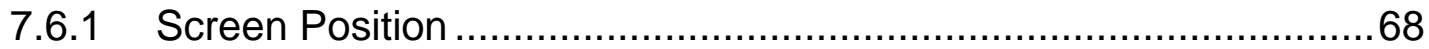

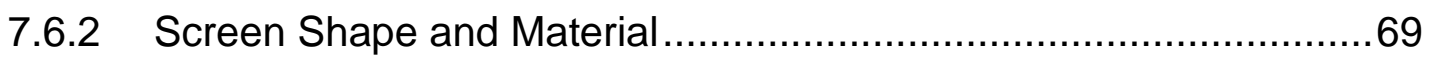

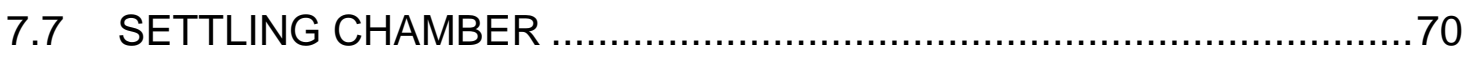

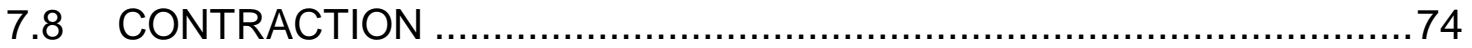

7.9 CONTRACTION RATIO SELECTION ................................................. 74

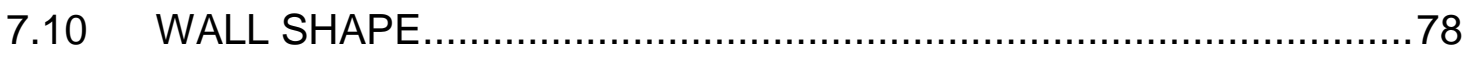

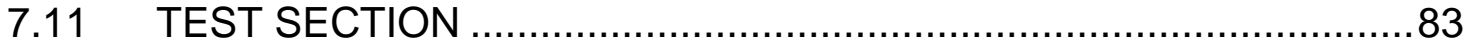

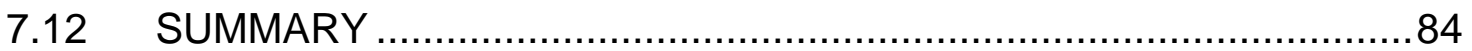

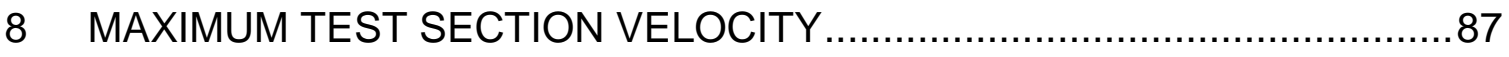

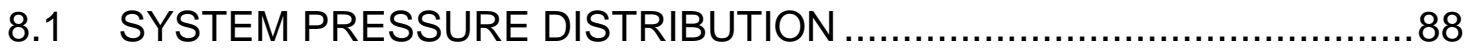

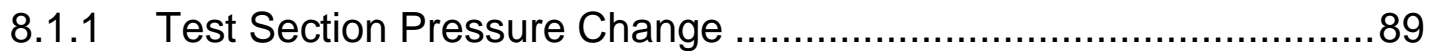

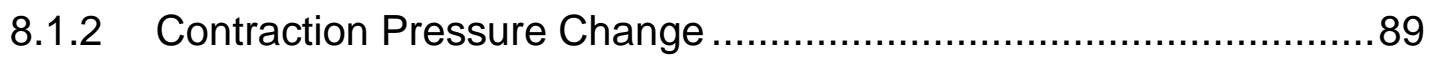

8.1.3 Settling Chamber Pressure Change ............................................ 90

8.1.4 Diffuser Pressure Change ............................................................

8.2 FAN WORKING POINT AT MAXIMUM POWER ….............................93

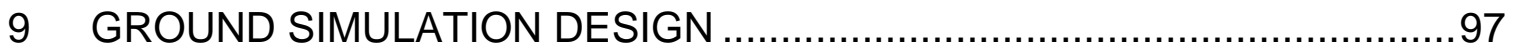

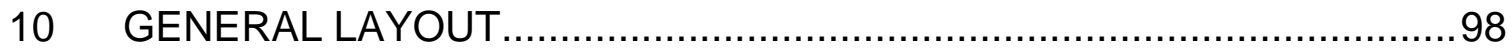

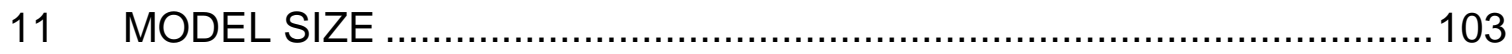

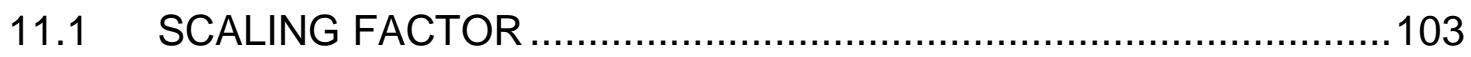

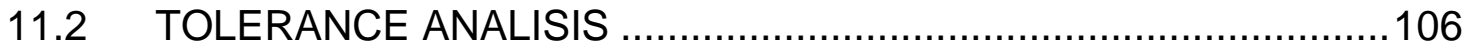

11.3 TEST AND MODEL RANGE VARIABLES ..................................107

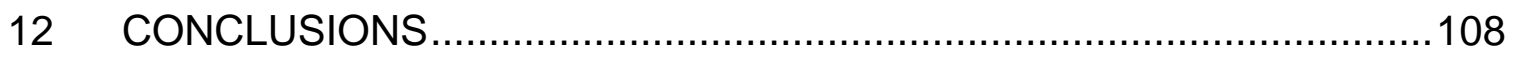

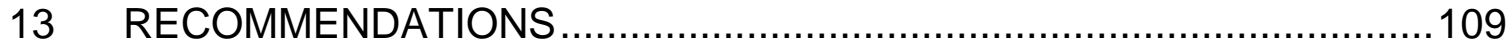

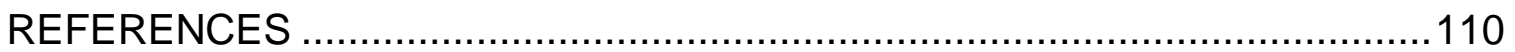

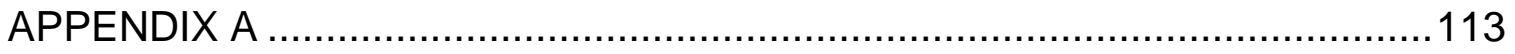

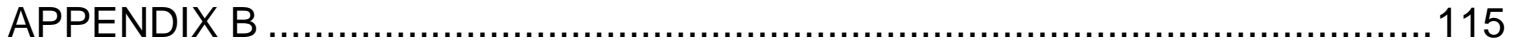

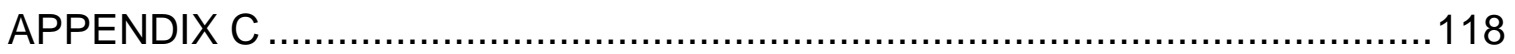

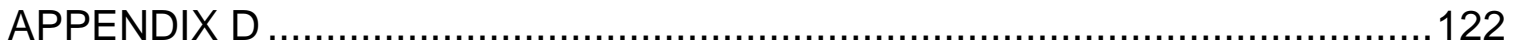

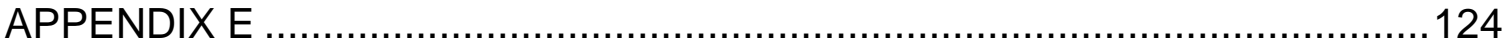




\section{LIST OF FIGURES}

Figure 1. Artistic representation of the Air-Ray prototype [Angle II, 2007] ................................. 2

Figure 2. Open return, tilting wind tunnel [Pennycuick, et al., 1997] ........................................ 8

Figure 3. Closed return, tilting wind tunnel [Pennycuick, et al., 1997] ...................................... 8

Figure 4. Free Flying model test [Wolowicz, et al., 1979]. ..................................................... 11

Figure 5. Values of the downwash correction factor for several aspect ratios [Schliestett, 1934] 15

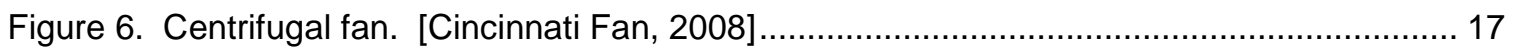

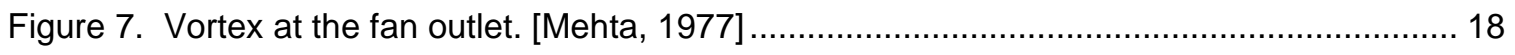

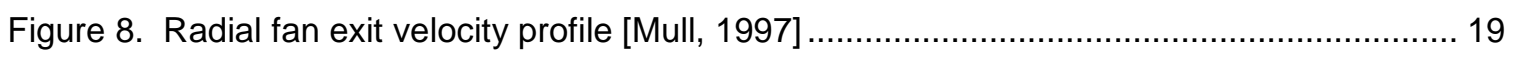

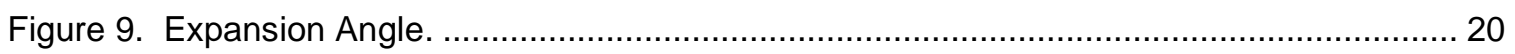

Figure 10. A vs. $2 \Theta$ for several wide angle diffusers [Mehta, 1977] ....................................... 22

Figure 11. Velocity Profile downstream of a screen [Mehta, 1985] ........................................... 24

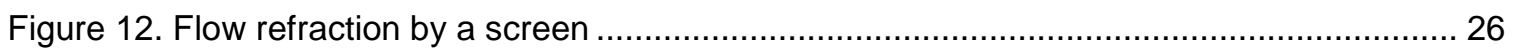

Figure 13. Screen profile and diffraction, curved and flat meshes .......................................... 27

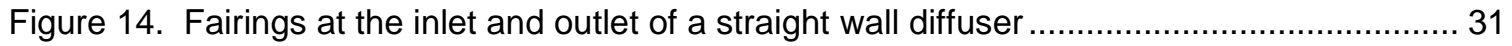

Figure 15. Vertical total pressure distribution at the diffuser exit, for several degrees of swirl

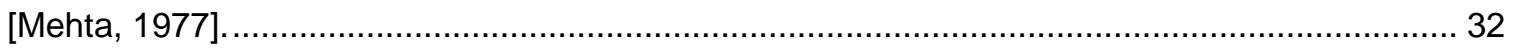

Figure 16. Horizontal total pressure distribution at the diffuser exit, for several degrees of swirl

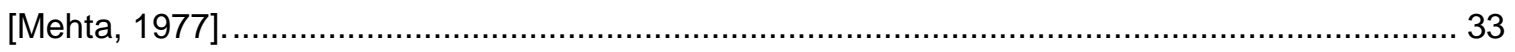

Figure 17. Variation in the Axial velocity non-uniformities in a contraction [Bradshaw, 1964] ..... 38

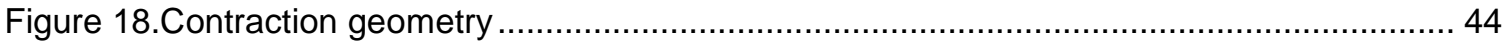

Figure 19. Differences between moving model or moving ground plane simulation and fixed

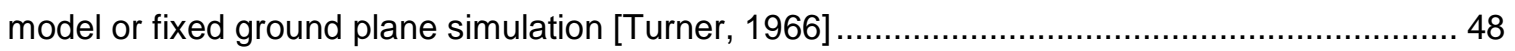

Figure 20. Selection of the ground simulation technique [Turner, 1966] ................................. 49

Figure 21. Influence of the belt velocity, $\mathrm{h} / \mathrm{b}=0.166, \boldsymbol{C L}, \boldsymbol{h}=\infty=\mathbf{7 . 4} \quad$ [Turner, 1966]............... 50

Figure 22. Boundary layer sucking at the belt [Turner, 1967] .............................................. 50

Figure 23. Testing of a wing in ground effect (WIG) vehicle [Seair Craft Inc., 2003] .................. 52

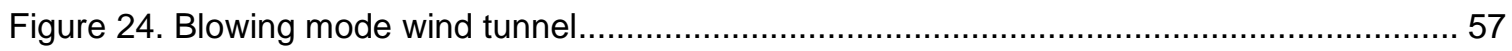

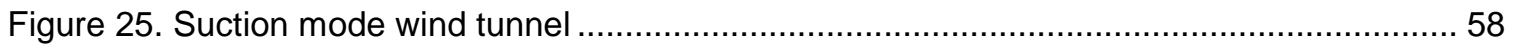

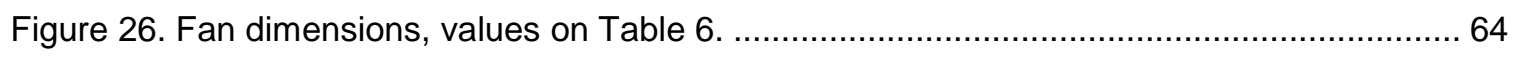

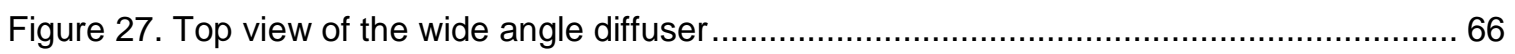

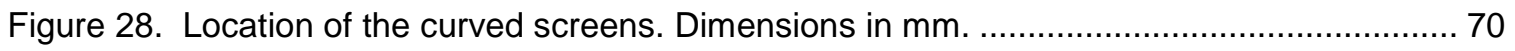

Figure 29. Screen and honeycomb position in the settling chamber. ....................................... 73

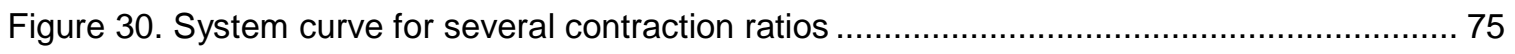

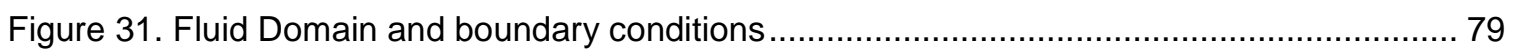

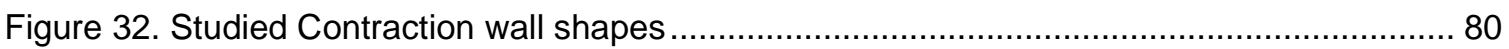


Figure 33. $u c r$ against $X p$ for the studied inlet velocities

Figure 34. $X$-velocity at the contraction exit, at planes $X Y$ and $X Z$ for $X p=0.46$ and an inlet velocity of $0.7 \mathrm{~m} / \mathrm{s}$....... 82

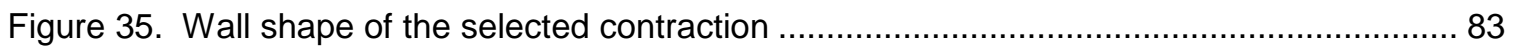

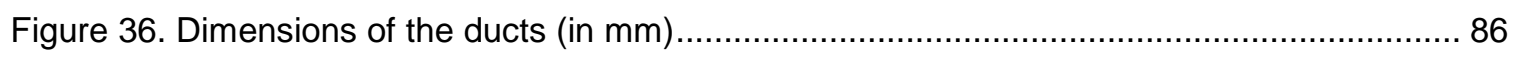

Figure 37. Schematic static and total pressure distribution along the wind tunnel. .................... 88

Figure 38. Fan and system pressure vs. volumetric flow curve (fan at max speed: 1750rpm).... 92

Figure 39. Static and Total pressure distribution along the wind tunnel for a test section velocity of

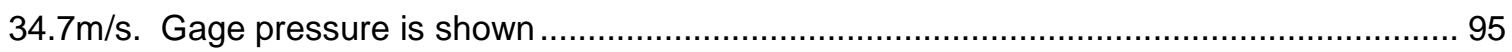

Figure 40. Fan power vs. volumetric flow curve (fan at max speed: $1750 \mathrm{rpm}$ ) ......................... 96

Figure 41. General Layout of the wind tunnel and its main components, horizontal position ...... 99

Figure 42. General Layout of the wind tunnel and its main components, tilted $50^{\circ} \ldots \ldots \ldots \ldots \ldots \ldots . . . . . .100$

Figure 43. Wind Tunnel Layout main dimensions, horizontal position ..................................... 101

Figure 44. Wind Tunnel Layout fan and ducts, exploded view. The diffuser is detailed in Figure 45. 102

Figure 45. Diffuser. Exploded view..... 102 


\section{LIST OF TABLES}

Table 1. Air Ray Prototype Specifications [Angle II, 2006], [Angle II, 2008] .................................. 2

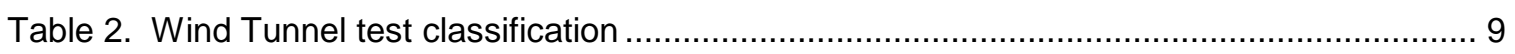

Table 3. Values for the pressure drop coefficient of a screen [Mehta, 1977] .............................. 29

Table 4. Test on ground effect used as a comparison cases.................................................. 52

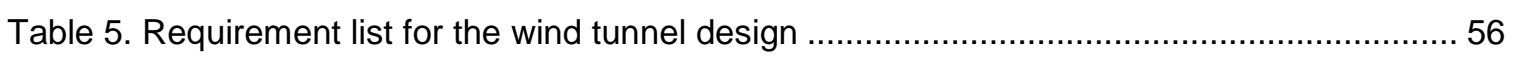

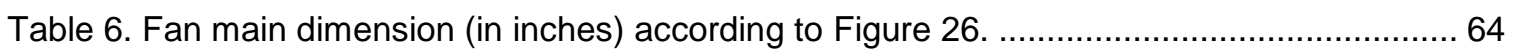

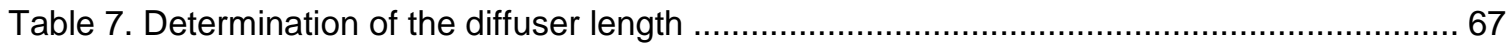

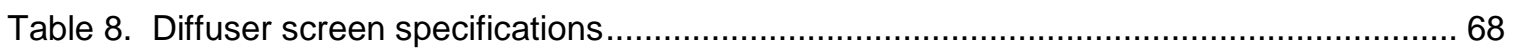

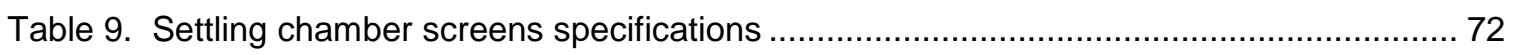

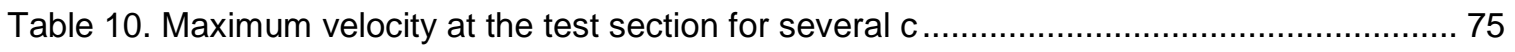

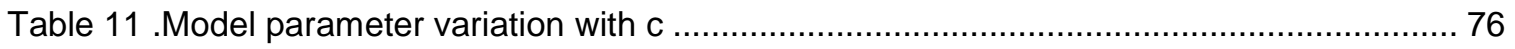

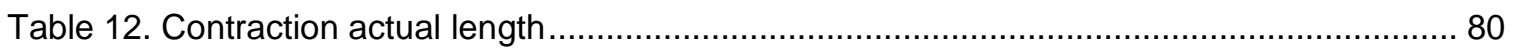

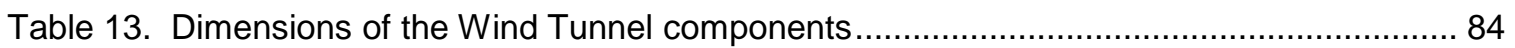

Table 14. Model and prototype Reynolds number, base on the vehicle length ........................ 106

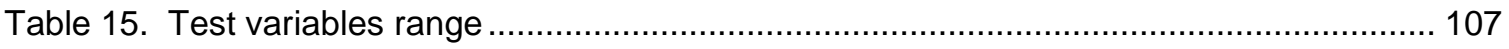




\section{NOMENCLATURE}

A: Diffuser area relation

$A_{c, e}$ : Cross-sectional area of the contraction exit $\left[\mathrm{mm}^{2}\right]$

$A_{c, i}$ : Cross-sectional area of the contraction inlet $\left[\mathrm{mm}^{2}\right]$

$A_{C S}$ : Area of the cross-section $\left[\mathrm{mm}^{2}\right]$

$A_{d, e}:$ Cross-sectional area of the diffuser exit $\left[\mathrm{mm}^{2}\right]$

$A_{d, i}$ : Cross-sectional area of the diffuser inlet $\left[\mathrm{mm}^{2}\right]$

$A_{m}$ : Frontal area of the model $\left[\mathrm{mm}^{2}\right]$

$A_{0}$ : Cross-sectional area of the test section $\left[\mathrm{mm}^{2}\right]$

$A_{p}$ : Frontal area of the prototype $\left[\mathrm{m}^{2}\right]$

$A_{s t c}:$ Cross-sectional area of the settling chamber $\left[\mathrm{mm}^{2}\right]$

$A_{S 1}$ : Cross-sectional area of the location of the first screen $\left[\mathrm{mm}^{2}\right]$

$A_{S 2}$ : Cross-sectional area of the location of the second screen $\left[\mathrm{mm}^{2}\right]$

$A R_{c, i}$ : Aspect ratio of the contraction inlet

$A R_{c, e}:$ Aspect ratio of the contraction exit.

$b$ : Vehicle span [m]

$c$ : Contraction ratio

$C_{p}$ : Total pressure coefficient

$C_{L, h=\infty}$ : Lift coefficient out of ground effect

$D_{c, i}:$ Square root of the contraction inlet area $[\mathrm{mm}]$

$D_{C S}:$ Cross-sectional diameter $[\mathrm{m}]$

$D_{H C}$ : Diameter of a circle with equal area to that of a honeycomb cell [m]

$d_{s c r}$ : screen wire diameter [mm]

$D P$ : Dynamic pressure $[\mathrm{Pa}]$

$D P_{f, e}$ : Dynamic pressure at the fan exit [Pa]

$D P_{f, i}:$ Dynamic pressure at the fan inlet $[\mathrm{Pa}]$

FanSP: Fan Static pressure [Pa] 
FanSP $P_{\text {max }}$ : Fan static pressure for $\dot{\forall}_{\text {max }}[\mathrm{Pa}]$

FanTP: Fan total pressure $[\mathrm{Pa}]$

Fr: Froude number

$g$ : Acceleration of gravity $\left[\mathrm{m} / \mathrm{s}^{2}\right]$

$h$ : Distance from the ground to the model [mm]

$H_{c, e}$ : Height of the contraction exit [mm]

$H_{c, i}$ : Height of the contraction inlet [mm]

$H_{c}(x)$ : Height of the contraction at location $\mathrm{x}[\mathrm{mm}]$

$H_{d, e}$ : Height of the diffuser exit [mm]

$H_{d, i}:$ Height of the diffuser inlet [mm]

$H_{0}$ : Height of the test section [mm]

$H_{s t c}$ : Height of the settling chamber [mm]

$K_{H C}$ : Pressure drop coefficient of the honeycombs

$K_{0, c}$ : Standardized pressure loss coefficient of the contraction

$K_{0, d}$ : Standardized pressure loss coefficient of the diffuser

$K_{0, s t c}$ : Standardized pressure loss coefficient of the settling chamber

$K_{s c r}$ : Screen pressure drop coefficient

$K_{s c r, d}:$ Pressure drop coefficient of the diffuser screen

$K_{s c r, s t c}$ : Pressure drop coefficient of the settling chamber screen

$K_{\text {sum }}$ : Summation of the $K_{s c r}$ in the diffuser

$L_{c}$ : Contraction length [mm]

$L_{c h}$ : Characteristic length of the vehicle [mm]

$L_{d}$ : Diffuser length [mm]

$L_{E D}$ : Length of the exit duct after the contraction [mm]

$L_{G r}$ : Full length of the moving ground [mm]

$L_{h}$ : Lift force at height $h[\mathrm{~N}]$

$L_{H C}$ : Length of the honeycomb cells [mm]

$L_{h=\infty}$ : Lift force out of ground effect [N]

$L_{m}$ : Model length [mm]

$L_{0}$ : Test section length [mm] 
$L_{p}$ : Prototype length $[\mathrm{m}]$

$L_{s t c}$ : Length of the settling chamber $[\mathrm{mm}]$

$M_{0}, M_{1}, M_{2}$ : Screen position non-dimensional parameters

$n$ : Number of screens

$n_{1}$ : Order of the polynomial of the first portion of the contraction.

$n_{2}$ : Order of the polynomial of the final portion of the contraction.

$p$ : Static pressure $[\mathrm{Pa}]$

$p_{\text {atm }}$ : Atmospheric pressure $[\mathrm{Pa}]$

$p_{f, e}$ : Static pressure at the fan exit $[\mathrm{Pa}]$

$p_{f, i}$ : Static pressure at the fan inlet $[\mathrm{Pa}]$

$p_{t}:$ Total pressure $[\mathrm{Pa}]$

$p_{t, f, e}:$ Total pressure at the fan exit $[\mathrm{Pa}]$

$p_{t, f, i}:$ Total pressure at the fan inlet $[\mathrm{Pa}]$

Re: Reynolds number

$r_{H C}$ : Length to diameter ratio of the honeycomb cells

$R P M_{m, \max }:$ Fan speed for the required velocity at the test section [rpm]

$R P M_{\text {max }}$ : Maximum fan speed (1750rpm)

$\langle\mathrm{u}\rangle$ : Mean velocity at the actual contraction exit [m/s]

$U_{c, e}$ : Velocity at the contraction exit [m/s]

$U_{c, i}:$ Velocity at the contraction inlet [m/s]

$\bar{u}_{c r}$ : Contraction exit non-dimensional velocity

$U_{f, i}$ : Fan inlet velocity $[\mathrm{m} / \mathrm{s}]$

$U_{m, \max }$ : Maximum required velocity at the test section $[\mathrm{m} / \mathrm{s}]$

$U_{0}$ : Test section velocity $[\mathrm{m} / \mathrm{s}]$

$U_{0, \max }$ : Maximum obtainable velocity at the test section $[\mathrm{m} / \mathrm{s}]$

$U_{v}$ : Vehicle velocity $[\mathrm{m} / \mathrm{s}]$

$u_{2 y, \max }$ : Maximum velocity in the $Y X$ plane, at the actual contraction exit [m/s]

$u_{2 y, \text { min }}$ : Minimum velocity in the YX plane (outside of the boundary layer), at the actual contraction exit $[\mathrm{m} / \mathrm{s}]$ 
$u_{2 z, \max }$ : Maximum velocity in the ZX plane, at the actual contraction exit [m/s]

$u_{2 z \text {,min }}$ : Minimum velocity in the ZX plane (outside of the boundary layer), at the actual contraction exit $[\mathrm{m} / \mathrm{s}]$

$V_{A I R}$ : Air velocity $[\mathrm{m} / \mathrm{s}]$

$V_{B E L T}$ : Belt linear velocity $[\mathrm{m} / \mathrm{s}]$

$\dot{W}$ : Fan power [watts]

$W_{c, e}:$ Width of the contraction exit [mm]

$W_{c, i}:$ Width of the contraction inlet [mm]

$W_{c}(x)$ : Width of the contraction at location $x[\mathrm{~mm}]$

$W_{d, e}:$ Width of the diffuser exit [mm]

$W_{d, i}:$ Width of the diffuser inlet [mm]

$W_{m}:$ Model span [mm]

$W_{0}$ : Test section width [mm]

$W_{p}$ : Prototype span [m]

$W_{s t c}$ : Width of the settling chamber [mm]

$x_{H C}$ : Distance between this screen and the honeycomb [mm]

$X_{p}$ :Ratio between the contraction length and the location of the points where the two polynomials overlap

$\alpha$ : Angle of attack

$\alpha_{s c r}$ : screen deflection coefficient

$\beta$ : screen open area ratio

$\delta$ : Velocity variation factor

$\delta_{c, e}:$ Velocity variation factor at the contraction exit

$\delta_{c, i}$ : Velocity variation factor at the contraction inlet

$\Delta p_{C}$ : Pressure change in the contraction $[\mathrm{Pa}]$

$\Delta p_{d}$ : Pressure change in the diffuser [Pa]

$\Delta p_{\text {fan }}$ : Required change in static pressure through the fan $[\mathrm{Pa}]$

$\Delta p_{\text {loss }}$ : Loss of pressure along a wind tunnel component [Pa]

$\Delta p_{0}$ : Pressure change in the test section $[\mathrm{Pa}]$ 
$\Delta p_{\text {Regain }}:$ Pressure regain in the diffuser $[\mathrm{Pa}]$

$\Delta p_{s c r}$ : Static pressure drop across the screen [Pa]

$\Delta p_{s t c}$ : Pressure change in the settling chamber [Pa]

$\Delta x_{i s, s t c}$ : Distance between the last screen and the contraction inlet [mm]

$\Delta x_{s, s t c}$ : Distance between the screens in the settling chamber [mm]

$\theta$ : Diffuser expansion half angle $\left[^{\circ}\right]$

$\lambda$ : Scaling factor

$\phi_{o}$ : Emergence angle of the flow approaching a screen [ $\left.{ }^{\circ}\right]$

$\phi_{i}$ : Incidence angle of the flow approaching a screen [ [0]

$\dot{\forall}:$ Volumetric flow in the wind tunnel $\left[\mathrm{m}^{3} / \mathrm{s}\right]$

$\dot{\forall}_{\max }$ : Maximum volumetric flow of the wind tunnel $\left[\mathrm{m}^{3} / \mathrm{s}\right]$

$\dot{\forall}_{m, \text { max }}$ : Volumetric flow for the required test section speed $\left[\mathrm{m}^{3} / \mathrm{s}\right]$

$\frac{1}{l}$ : Number of opening per inch in the screen $\left[\right.$ inch $\left.^{-1}\right]$

$\frac{1}{2} \rho U_{s c r}^{2}$ : Dynamic pressure of the flow approaching perpendicularly to the screen $[\mathrm{Pa}]$ 


\section{INTRODUCTION}

In the development of a new aircraft, modeling the operation before construction is a crucial step. At present, technological advancements with computers, computational simulations have become a very practical and efficient approach to modeling aircraft. Its advantages include that it's quick, it's inexpensive, and it's easy to make modifications to the model. Nevertheless, this approach has not overcome the needs for a traditional experimental approach, partly because there are complex phenomena associated with an aircraft's operation that current computational methods are not capable of modeling. Thus, results obtained from the computational methods often require comparison against existing experimental results in order to be validated. These two methods have therefore become complementary and are frequently used together.

In vehicles where the operation involves a fluid flow (in this case air), the above mentioned complex phenomena can occur. Therefore, an experimental approach is very useful in understanding the flow characteristics. In the application of this approach, a wind tunnel is a basic simulation tool to approximately recreate the operational conditions.

The goal of this study is to design a wind tunnel capable of simulating the operational conditions of a wing in ground effect vehicle called Air-Ray, shown in Figure 1 [Angle II, 2007]. This vehicle is a gravitationally powered recreational

glider that flies down slopes. This craft is designed to operate only in ground effect, at about $1 \mathrm{~m}$ above the surface. Its control surfaces are a pair of ailerons, flaps, spoilers and a rudder. Table 1 lists the specifications of the Air-Ray prototype. 


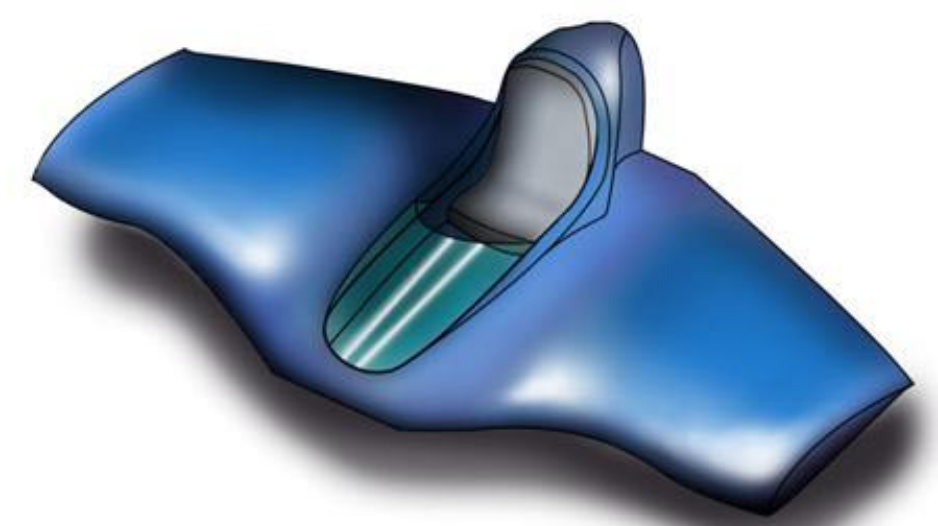

Figure 1. Artistic representation of the Air-Ray prototype [Angle II, 2007]

Table 1. Air Ray Prototype Specifications [Angle II, 2006], [Angle II, 2008]

\begin{tabular}{|l|c|c|}
\hline \multicolumn{3}{|c|}{ Air-Ray Prototype Specifications } \\
\hline Length, $L_{p}$ & $8 \mathrm{ft}$ & $2.44 \mathrm{~m}$ \\
\hline Width (Span), $W_{p}$ & $13 \mathrm{ft}$ & $3.96 \mathrm{~m}$ \\
\hline Maximum speed (relative to ground) & $80 \mathrm{ft} / \mathrm{s}$ & $24.4 \mathrm{~m} / \mathrm{s}$ \\
\hline Stall speed & $23.5 \mathrm{ft} / \mathrm{s}$ & $7.2 \mathrm{~m} / \mathrm{s}$ \\
\hline Frontal Area, $A_{p}$ & $23.89 \mathrm{ft}{ }^{2}$ & $2.22 \mathrm{~m}^{2}$ \\
\hline Air speed (Updraft) & $0-13.8 \mathrm{ft} / \mathrm{s}$ & $0-4.2 \mathrm{~m} / \mathrm{s}$ \\
\hline Wing Area & $190.5 \mathrm{ft}$ & $17.7 \mathrm{~m}^{2}$ \\
\hline Total Weight (including rider) & $375 \mathrm{bf}$ & $170 \mathrm{kgf}$ \\
\hline Empty Weight & $125 \mathrm{lbf}$ & $57 \mathrm{kgf}$ \\
\hline Flight altitude & $1-4 \mathrm{ft}$ & $0.3-1.2 \mathrm{~m}$ \\
\hline Estimated Lift Coefficient (in ground effect) & \multicolumn{2}{|c|}{2.0} \\
\hline Estimated Drag coefficient (in ground effect) & \multicolumn{2}{|c|}{1.1} \\
\hline Froude number (Fr) & \multicolumn{2}{|c|}{$3.99 \times 10^{6}$} \\
\hline Reynolds number (Re) & \multicolumn{3}{|c|}{} \\
\hline
\end{tabular}

To have the ability to investigate the effects of gravity, aerodynamic forces and proximity of the ground on the flight dynamics and stability of this vehicle, it is desirable that the wind tunnel can use free-flying models. This requires a 
wind tunnel with tilting and a moving ground plane capability. With such a wind tunnel it can be possible to investigate the behavior of the glider when flying and to understand its stability and control characteristics under several external conditions (varying slope angle, wind speed, vehicle weight, etc.). Because the model size and the scaling factor are unknown (though prototype specifications are known), it is also an objective of this work to find these values.

This study is limited to the aerodynamic design of the wind tunnel which involves the sizes and shapes of the wind tunnel ducts, the flow conditioners, and the ground plane simulation. The test section instrumentation, model holding system, tilting system and structural system will not be treated in this study, however a general layout of the wind tunnel will be provided.

Before this study, a prototype wind tunnel was constructed [Hubbell, et al. 2008] to evaluate these requirements but velocity measurements in the test section showed a strong non-uniformity of the velocity profiles including some areas of backflow. That prototype wind tunnel uses a centrifugal fan connected directly to a diffuser to blow air into the test section. Several screens have been used between the fan outlet and the test section to try to homogenize the flow velocity. The ground was simulated using a moving belt. The results obtained with the prototype wind tunnel validated the ground plane design, the blower and the instrumentation, but a new wind tunnel is required due to the non-uniformities in the test section.

It is strongly desired that the new design reuses parts of the prototype wind tunnel, especially the centrifugal fan and the moving belt. It is also important to note that this new wind tunnel will be located at WVU hangar.

Taking these constraints into account, a list of requirements will be made and shall be the start point of the wind tunnel design process. 


\section{METHODOLOGY}

To obtain a design of a wind tunnel that fulfills the requirements two basic configurations were analyzed and then evaluated: a blowing mode wind tunnel and a suction mode wind tunnel. The blowing mode wind tunnel was chosen according to evaluation parameters such as construction effort and flow uniformity at the test section. The chosen configuration was then designed according to the method proposed by Mehta (1977) and Mehta, et al. (1979), which is an entirely experimental method and is based on several successful wind tunnel designs. Mehta (1977) and Mehta, et al. (1979) study each main component of the wind tunnel: fan, diffuser, settling chamber, flow conditioners, contraction and test section. The following is a brief description of each one of these components, including an explanation of how they were designed in the present study.

Fan. The fan is the component that generates the air current in the wind tunnel. The method proposed by Mehta (1977) recommends the use of centrifugal fans blowing air in to the test section. This is not a component to be designed rather Mehta (1977) offers recommendations for its selection from industrial fan models. For this case it is desired to use the fan of the prototype wind tunnel that was constructed before.

Wide-angle diffuser. The function of this component is to create a low velocity region (settling chamber) where the flow conditioners can be located, the low velocity is needed to minimize the pressure losses at the flow conditioners. The wide angle diffuser is a component that requires a very careful design in order to maintain a uniform flow across the test section. This component was designed using the method proposed by Mehta (1977). 
Settling chamber. This component holds the flow conditioners such as honeycombs and screens. The component has the lowest velocity in the wind tunnel in order to minimize the pressure losses across the flow conditioners. This component was designed using recommendations given by Mehta (1977).

Flow conditioners. Honeycombs and screens are used to improve the quality of the flow across the test section. Honeycombs are used to straighten the flow (to have uniform direction of the velocity) and screens are used to encourage a uniform velocity (to have uniform magnitude of the velocity). These components were designed using the method proposed by Mehta (1977).

Contraction. This component is needed to further improve the quality of the flow, it reduces the mean and fluctuating velocity variations. To design the contraction the method proposed by Su (1991) was used, since it presents more design information than Mehta's method. With the method proposed by Su several options for the contraction wall shape were obtained, with the aid of CFD simulations the option that produces the most uniform flow across the test section was chosen.

Test Section. At this component is where the model is located. It is very important to have a uniform flow velocity across and along this component. To design this component, recommendations of several references were used, such as [Mehta, 1977], [Mehta, et al., 1979], [Barlow, et al., 1999], [Heyson, 1971] and [Schliestett, 1934]. A special requirement for this study is that the test section has to have a ground simulator. This special element was designed using recommendations proposed by Turner $(1966,1967)$ and Brown (1939) which studied the testing of vehicles in ground effect. The shape of the test section and the width to height ratio were chosen to account for the correction factors in the wind tunnel test results, available construction techniques, time and cost. 
Knowing the dimensions of the test section, the model dimensions and the scaling factor were found using the blockage factor and the prototype specifications. The required test velocity was obtained using the scaling factor and the scaling parameter (Froude number).

Having all the components designed the pressure losses along the wind tunnel were calculated, and the flow vs. pressure curve for the system was obtained, comparing this curve with fan flow vs. pressure curve (provided by the fan manufacturer [Cincinnati Fan, 2008]), the fan working point and the maximum velocity at the test section were obtained. Since the maximum obtained velocity was larger than the required test velocity, the fan of the prototype wind tunnel will work in the current design. 


\section{LITERATURE REVIEW}

This chapter deals with a general description of wind tunnels, wind tunnel components and wind tunnel tests according to the information available in the technical literature. This information will be needed in the design process.

\subsection{WIND TUNNEL DESCRIPTION AND STATE OF THE ART}

There are two basic wind tunnel configurations: open return, Figure 2 [Pennycuick, et al., 1997], where at some point downstream of the test section the flow is discharged to the atmosphere or room where the wind tunnel is located, and closed return, Figure 3 [Pennycuick, et al., 1997], where the fan inlet is connected with the test section exit by a diffuser, and the air is continuously recirculated. The advantages of open return are that they are smaller and easier to construct compared to a closed return wind tunnel with the same test section size and maximum speed. Closed return wind tunnels require less power to operate, and there is more control over the flow conditions in the test section, and are less noisy to operate [Barlow, et al., 1999]. Open return wind tunnel are usually recommended for small low speed wind tunnels, which are tunnels with a test section, cross-sectional area of less than $0.5 \mathrm{~m}^{2}$ and with free stream velocities of less than $40 \mathrm{~m} / \mathrm{s}$ [Bell, et al., 1988].

A tilting wind tunnel is a special purpose wind tunnel, normally used in the study of the flight of birds (in steady flight, not wing flapping), and can be open or closed return, as is shown in Figure 2 [Pennycuick, et al., 1997] and Figure 3 [Pennycuick, et al., 1997], respectively. These tunnels normally have small angles of inclination. The wind tunnel of Figure 3 [Pennycuick, et al., 1997] can be tilted about $8^{\circ}$ descent and $6^{\circ}$ climb. For the current study the maximum desired inclination angle is $50^{\circ}$. 


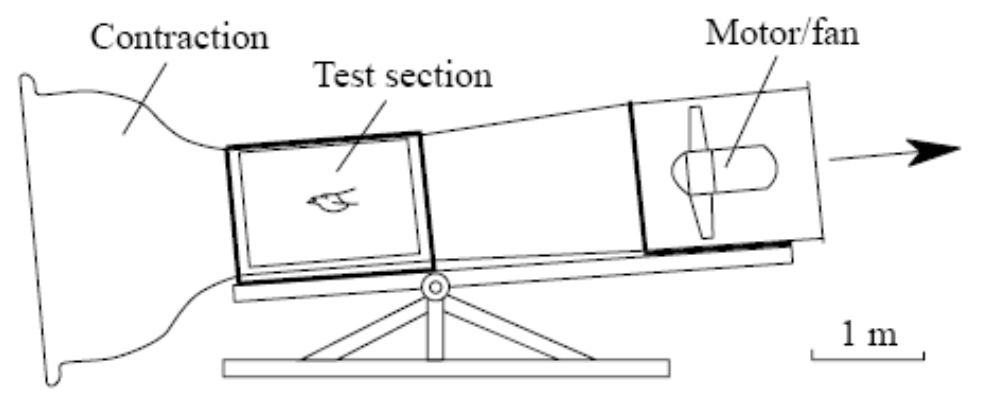

Figure 2. Open return, tilting wind tunnel [Pennycuick, et al., 1997].

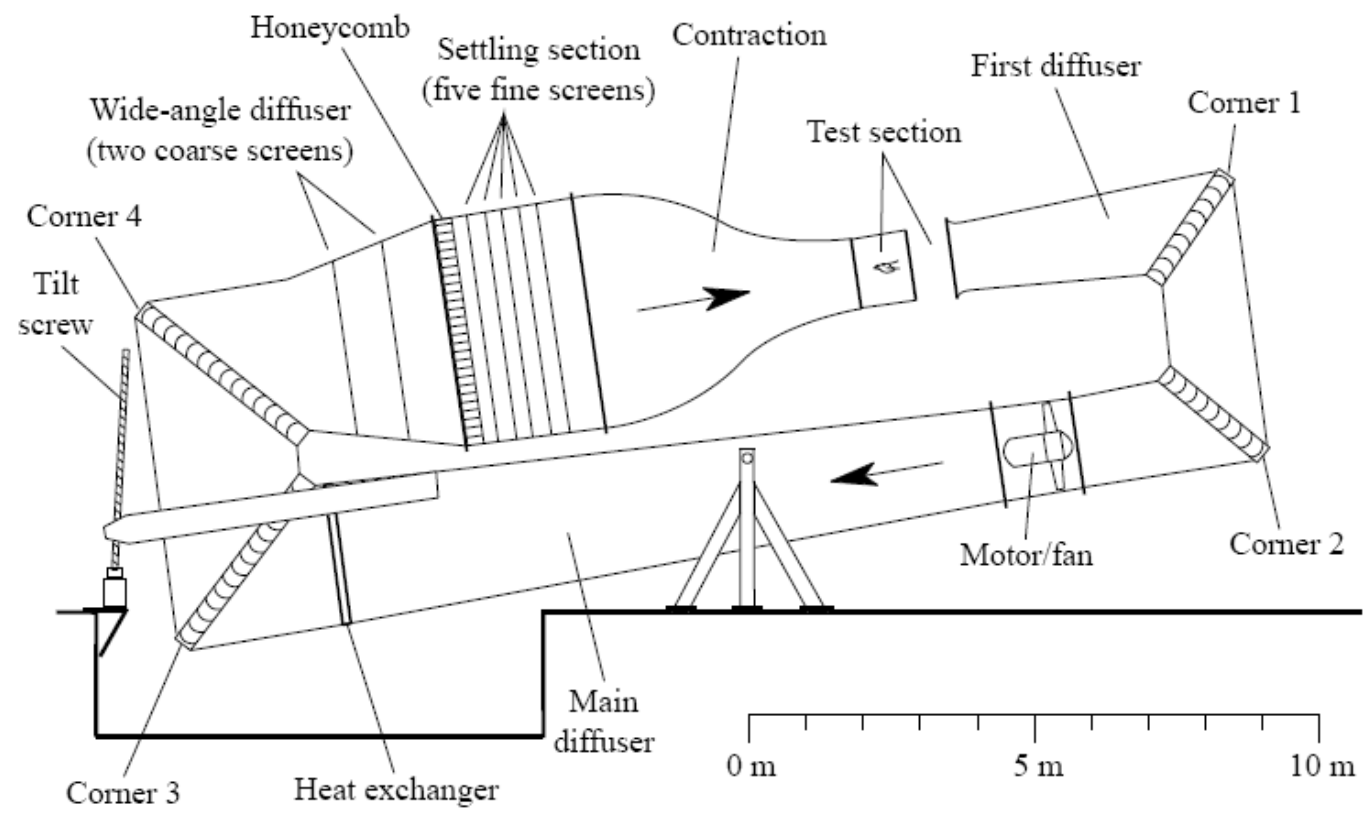

Figure 3. Closed return, tilting wind tunnel [Pennycuick, et al., 1997].

\subsection{WIND TUNNEL MODELS}

There are different types of tests that can be performed in a wind tunnel; each test has its own objectives and restrictions, and usually a specific type model. Barlow, et al., (1999) and Wolowicz, et al., (1979) classify the different models that are used in wind tunnel tests with the most relevant shown on Table 2. 
Table 2. Wind Tunnel test classification

\begin{tabular}{|c|c|c|c|}
\hline & & Type of model & Test Conditions \\
\hline \multirow{2}{*}{\multicolumn{2}{|c|}{$\begin{array}{l}\text { Mounted Wind Tunnel } \\
\text { Models }\end{array}$}} & \multirow[t]{2}{*}{ Rigid Models } & Steady-State Test \\
\hline & & & Dynamic Test \\
\hline & & $\begin{array}{l}\text { Aeroelastic } \\
\text { Models }\end{array}$ & Dynamic Test \\
\hline \multirow[t]{3}{*}{$\begin{array}{l}\text { Free- } \\
\text { Flying } \\
\text { Models }\end{array}$} & $\begin{array}{l}\text { Spin- } \\
\text { tunnel } \\
\text { models }\end{array}$ & Rigid model & Dynamic Test \\
\hline & \multirow{2}{*}{$\begin{array}{l}\text { Wind } \\
\text { Tunnel } \\
\text { free flying } \\
\text { models }\end{array}$} & Rigid model & Dynamic Test \\
\hline & & Rigid model & Steady-State Test \\
\hline
\end{tabular}

\subsubsection{Mounted Wind Tunnel Models}

A model that is fixed to a balance that measures the forces and moments acting on it, and that sometimes potentially have several pressure ports to obtain the static pressure distribution on their surface. Since the model is fixed the gravity has no effect on the model behavior and therefore the Froude number does not have to be considered [Barlow, et al., 1999], [Wolowicz, et al., 1979] and for incompressible flow the Reynolds number is conserved (or as close as possible) between the model and the prototype.

For static tests the measurements are taken with constant external conditions (air velocity, control surfaces, etc), then these conditions are changed and new measurements are taken and so on. This obtains the parameters of interest for several Reynolds numbers, angles of attack, and control surface positions.

For dynamic tests one or more of the variables are changing, usually the model is rotating or oscillating [Wolowicz, et al., 1979], or its control surfaces are moved, where the objective of the test is to measure the response of the model to these changes, providing the values of some of the parameters related to the dynamics effects (for example the derivatives of the moment coefficients).

For rigid models the parameters of interest are usually the lift, drag and moment coefficients. In Aeroelastic models the parameters of study are related 
to the aeroelastic phenomena in the model and its flutter characteristics, and how this affects the performance, stability and control of the vehicle [Wolowicz, et al., 1979].

\subsubsection{Free Flying Models}

This type of model is envisioned as that which will be used in the wind tunnel developed in the present work. These models are not fixed to the wind tunnel, and for incompressible flow the most important parameter is the Froude number. This non-dimensional number has to be the same for the model and the prototype of the vehicle being studied to adequately simulate the desired phenomena.

Free flying wind tunnel models are rigid models, they can be powered (capable of thrust), with operable control surfaces, and with the desired instrumentation, according to the objectives of the test. For example they can be equipped with pressure transducers and accelerometers (to measure both linear and angular accelerations). It is recommended to test these models at several air velocities, vehicle configurations and angles of attack. The model is controlled remotely by one or more operators, and it is held by a safety cable to restrain it when the test finishes or if there is a loss of control of the model. Figure 4 [Wolowicz, et al., 1979] shows a representation of a free flying test being conducted.

Spin-tunnel models are used to study how an aircraft recovers from a spin, in these tests an unpowered spinning model is thrown in to the test section of a spin tunnel. The model behavior is recorded during the test; usually they do not have instrumentation. 


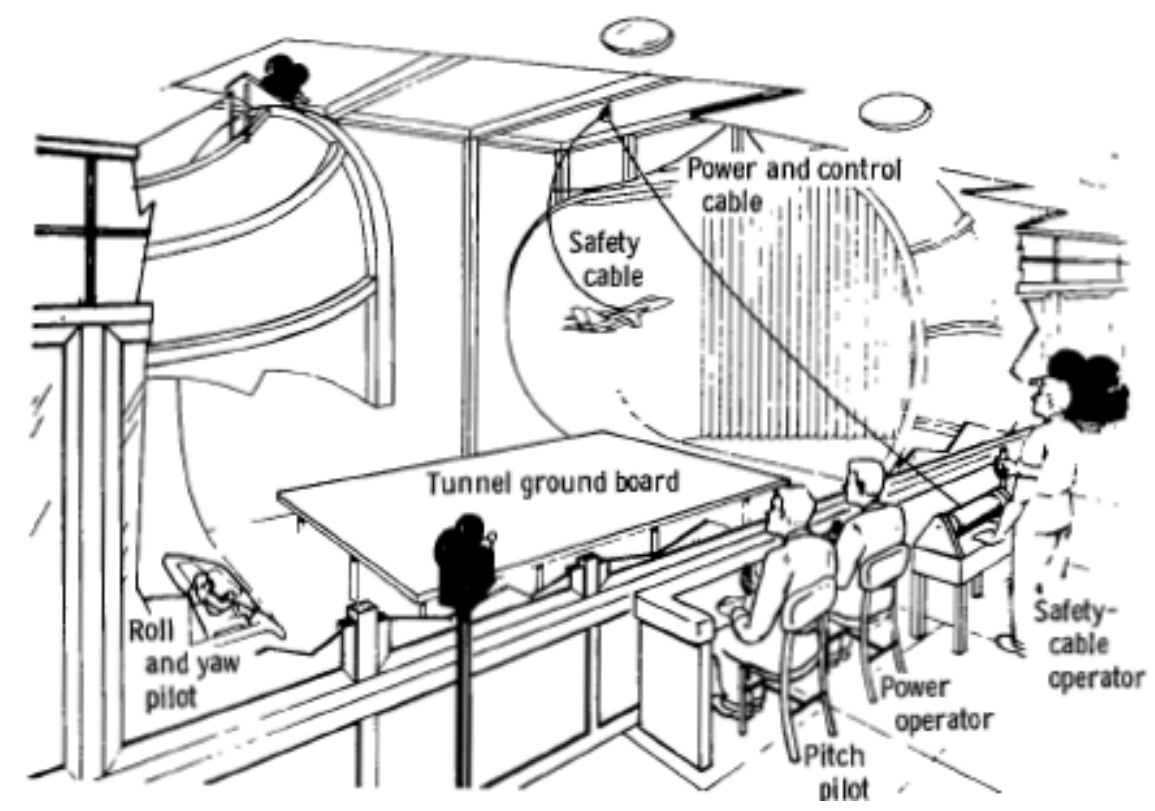

Figure 4. Free Flying model test [Wolowicz, et al., 1979].

According to Wolowicz, et al. (1979), free flying model tests are qualitative studies which have the objective of evaluating the dynamic stability and control characteristics for several angles of attack, including stall.

The tests can be steady, when the model is maintained at a constant flight condition (nothing change during the test, same velocity, control surface configuration, etc), or dynamic when the response to a parameter change is the objective of the study, for example, a control surface is moved during the test.

Since Air-Ray is an unpowered vehicle (the drag is overcome by the component of the gravitational force that is parallel to the slope), the free flight model only has control surfaces, and the tests need to be performed for several slope angles, velocities, and positions of the control surfaces.

\subsection{TESTING PARAMETERS}

In order to model the flow correctly around a vehicle having geometrical similitude it is also necessary to conserve some parameters between the flow in the model and the real flow, i.e. the flow around the prototype. These 
parameters, called testing parameters or similarity parameters are ratios between the forces acting on the fluid. The three most frequently used parameters in aerodynamics are: Reynolds number, Mach number and Froude number.

When free flying models are used the parameter that has to be conserved is the Froude number, Fr [Wolowicz, et al., 1979], [Barlow, et al., 1999], defined in Eq. 1 , where $U_{v}$ is the velocity of the vehicle, $L_{c h}$ is a characteristic length of the vehicle and $g$ is the acceleration of gravity.

$$
F r=\sqrt{\frac{U_{v}^{2}}{L_{c h} \cdot g}} .
$$

This non-dimensional number represents the square root of the ratio between inertia forces and gravity forces. This parameter is important for the AirRay since the gravity force is the driving force, or the force that generates the thrust required for the model to fly down the slope; and the inertial forces, are also important, since they are the forces that generate the aerodynamic reactions over the vehicle and thus support it.

The Reynolds number is also a very important parameter in the flow around the Air-Ray, since it can affect the flow pattern over its surface (laminar or turbulent). Unfortunately the only way to conserve simultaneously, the Froude and Reynolds number is by having a full scale model; this is valid when testing with the same fluid at the same properties (temperature, pressure) for the model and the prototype.

Since a full scale simulation implies high costs, it is possible to test at lower Reynolds numbers and useful results can be obtained [Paulson, 1957]. Even if the Reynolds number is not matched, it is helpful to conserve the transition points where the boundary layer changes from laminar to turbulent between the model and the prototype [Barlow, et al., 1999], [Wolowicz, et al., 1979]. These transition points can be obtained studying the flow in fixed models, and matching the Reynolds numbers. Then, to force the transition in the free flying model, its roughness is modified near the desired point, this can be 
achieved with tapes, paints, wires, etc [Barlow, et al., 1999]. Forcing the transition is possible since the transition occurs earlier (toward vehicle nose) in the prototype than in the model.

\subsection{WIND TUNNEL COMPONENTS}

\subsubsection{Test Sections}

The following section describes the most important factors of a test section, such as cross-sectional shape, aspect ratio, test section type (open or closed), test section length, instrumentation, flexibility and model mounting system.

\subsubsection{Cross-Sectional Shape of the Test Section.}

A wind tunnel can have different cross-sectional shapes in its ducts, for example when a square test section is required and an axial fan is used. Usually for ease of construction it is better to have a rectangular cross-sectional shape in all the components of the wind tunnel, in the present case the fan outlet has a rectangular cross-section, so it will be helpful to maintain this shape along the wind tunnel length (although the aspect ratio can change along the wind tunnel, and maintaining ease of construction).

Wind tunnels with rectangular cross-sections and in general with non axisymmetric cross-sections have some problems related with the flow quality at the test section: low velocity regions at the corners and cross flow at the contraction, which increases the tendency of the boundary layer to separate at the contraction, as discussed in see section 3.4.5. So circular and octagonal cross-sectional shapes are often used to reduce the influence on flow quality. In this aspect, the most advantageous shape is the circular one, due to the fact that there are no corners and the associated low velocity regions. Also, a contraction with circular cross-section does not have cross flow problems due to its axisymmetry. The disadvantage of a circular test cross-section is that they are 
difficult to construct, in particular the contraction. A rectangular section is very easy to construct, but has the problems described above. An octagonal section is a compromise of the circular and rectangular sections, with a smoother air flow than the rectangular cross-section and easier to build than the circular crosssection, but it also has some of the disadvantages.

Nevertheless a well designed wind tunnel with a rectangular test section can have a flow in the test section with adequate flow quality. Mehta (1977), Bell, et al. (1988) and Su (1991) propose methods for designing wind tunnels and wind tunnel components, with rectangular test section, and affirm that the obtained flow uniformity can be less than $\pm 0.5 \%$ variation outside the boundary layers, which is adequate for typical aeronautical applications [Mehta, et al., 1979].

\subsubsection{Aspect ratio.}

Sometimes the purpose of a wind tunnel can define the value of the aspect ratio of its test section (width over height), besides aeronautical wind tunnels, there are wind tunnels intended for testing automobiles, buildings, and special purpose tunnels such as boundary layer tunnels, climatic wind tunnels, vertical wind tunnels, etc. The purpose and objectives of a test can change the required aspect ratio, sometimes it is due to the studied problem itself (as an in boundary layers studies) and sometimes it because an optimal value exists, as when testing wings, where a value of 1.5 is suggested in order to minimize the downwash correction factor, as can be see in case I of Figure 5 [Schliestett, 1934]. Due to this and the frequent uses of wind tunnels for aeronautics, wind tunnels with aspect ratios between 1.4 and 1.5 are very popular [Mehta, 1977], [Barlow, et al., 1999], and for facilitating the measures the larger dimension is located horizontally. 


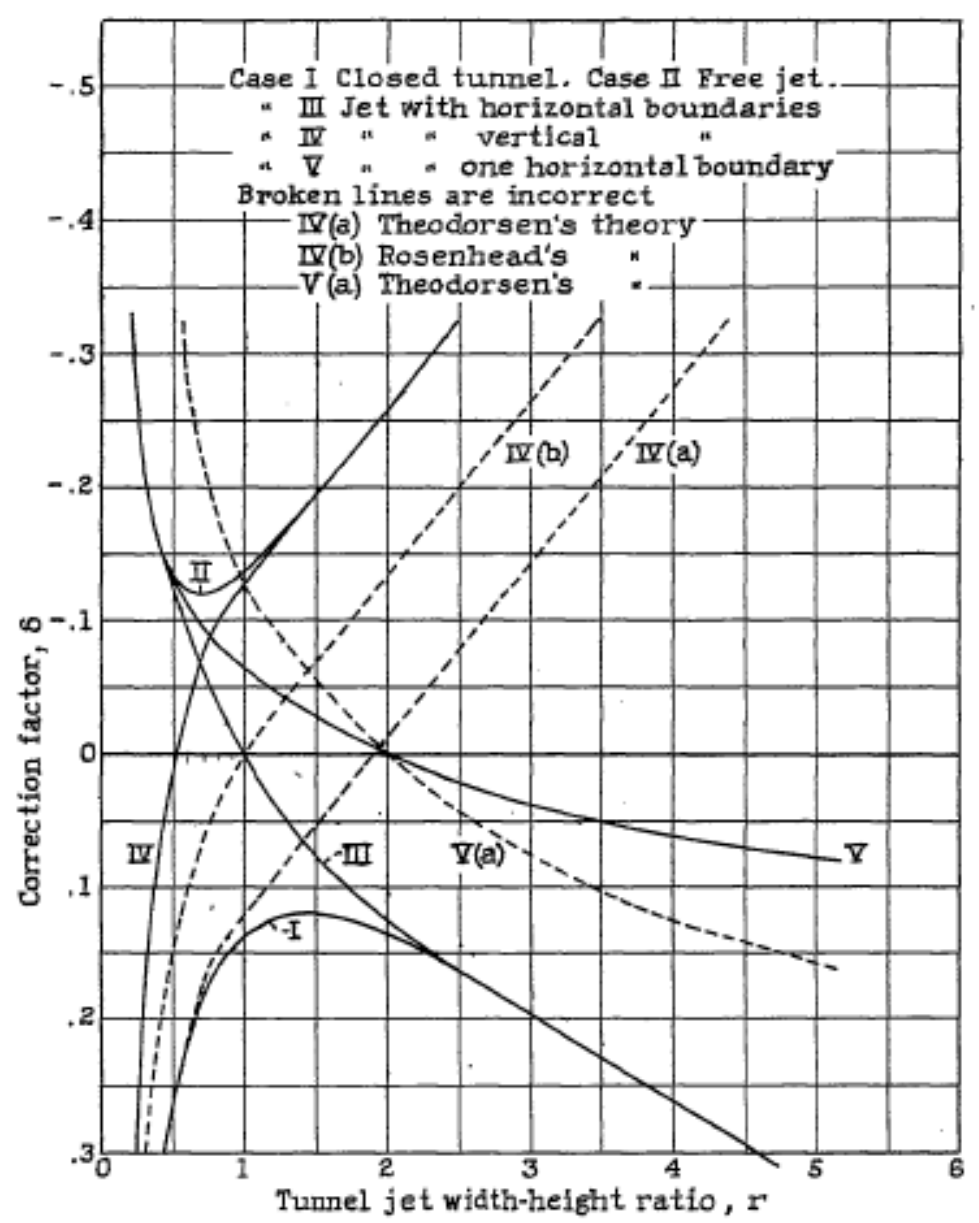

Figure 5. Values of the downwash correction factor for several aspect ratios [Schliestett, 1934]

\subsubsection{Open and Closed Test Sections.}

A closed test section is surrounded entirely by walls, and an open test section is when one or more of the walls are absent. The main advantage of an open test section is that it facilitates: taking measurements in the flow and in the model, mounting/unmounting the model in the test section and using flow visualization techniques. Another important advantage is that the pressure drop along the test section and other wall effects are negligible (see section 3.5 ).

One of the problems when using an open test section with an open return wind tunnel is that it usually has to be surrounded by a sealed-off chamber to avoid disturbances in the open jet. In a closed return wind tunnel this is not required since the air flow tends to follow the wind tunnel path due to the suction 
of the return diffuser, and according to Heyson (1971) a smooth flow without pulsations can be obtained more easily in a closed test section, with a practically zero longitudinal static pressure drop.

\subsubsection{Test Section Length}

The recommended length of the test section is between 1 to 1.5 times its width [Barlow, et al., 1999]. Since the boundary layer grows along the test section, this reduces its effective cross-sectional area and this increases the velocity. Since outside the boundary layer the total pressure is conserved then a drop in the static pressure will occur. This can affect the behavior of the model, because due to this difference of pressures an additional drag force will appear (called horizontal buoyancy). To avoid this it is necessary to have an increment in the cross-sectional area along the test section. This can be done by tapering the fillets in the corners, i.e. the width of the fillets have to decrease along the duct, or sometimes tapering the walls, similar to a diffuser with a very small expansion angle. In the last case, it is recommended to start with an angle of about $1^{\circ}$, and by trial and error make finer adjustments, to find the appropriate value for this angle. These finer adjustments can be done by filling the corners.

Mehta (1977) recommends installing the test section at a distance of 0.5 diameters after the contraction to reduce the non uniformities of the flow that comes out of the contraction.

\subsubsection{Instrumentation}

The present work does not finalize instrumentation; nevertheless a system to measure the volumetric flow (usually using manometers) and the belt tangential speed is needed to describe the test section conditions. For the wind tunnel tuning and calibration is also required to have the possibility to use pitot tubes, rakes, and hot wire anemometers. 


\subsubsection{Flexibility}

To have a more flexible design a detachable test section is often used, in order to study the flow over different devices, and test section shapes. For example round test sections can be used or two-dimensional test sections for boundary layer studies.

\subsubsection{Model Mounting}

Since free flight models are being considered it is necessary to hold the model to avoid it falling into the settling chamber or to stop it from blowing out of the tunnel due to uncontrolled motion or when the test is finished.

\subsubsection{Fans}

Mehta (1977) and Mehta, et al. (1979) study and recommend the use of centrifugal blowers (Figure 6 [Cincinnati Fan, 2008]) for low speed wind tunnels. The backward-facing aerofoil type is specifically recommended and commercially available industrial fans are adequate, making it unnecessary to design and construct a special fan for operating the wind tunnel. Some of the advantages of these fans compared to axial fan, when used in wind tunnels, are that their operation is more steady, efficient and is less affected by the conditions at the entrance and at the test section (while operating as a blower).

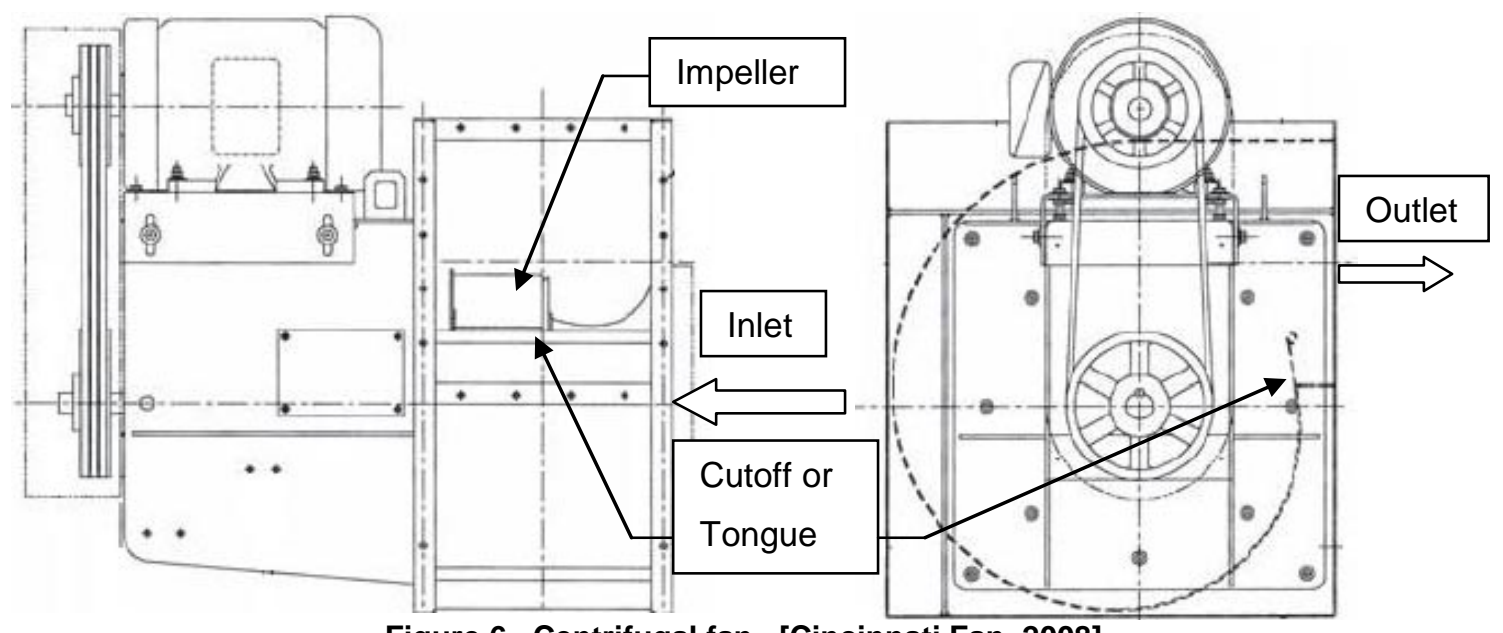

Figure 6. Centrifugal fan. [Cincinnati Fan, 2008] 
Mehta (1977) reports that wind tunnels operating with this type of fans can have fairly uniform velocity profiles ( $0.2 \%$ variation on total pressure) and low turbulence intensity levels (less than $0.1 \%$ ) at the test section, if they are appropriately designed.

As it is known at the exit of radial fans there is vortex-type flow, due to the asymmetric position (see Figure 6 [Cincinnati Fan, 2008]) of the impeller which causes the flow to deflect toward the "empty" regions in the volute (see Figure 7, [Mehta, 1977]). Also, as can be seen in Figure 8 [Mull, 1997], the flow is separated at the cutoff (also known as splitter plate or "tongue" see Figure 6 [Cincinnati Fan, 2008]), which is the element that separates the air that rotates in the volute with the impeller from the air that comes out of the fan. These phenomena generate irregularities and unsteadiness in the flow at the fan exit that have to be reduced to an acceptable level in the settling chamber and in the contraction in order to have the required flow conditions at the test section (sections 3.4.4 and 3.4.5 describes these components).

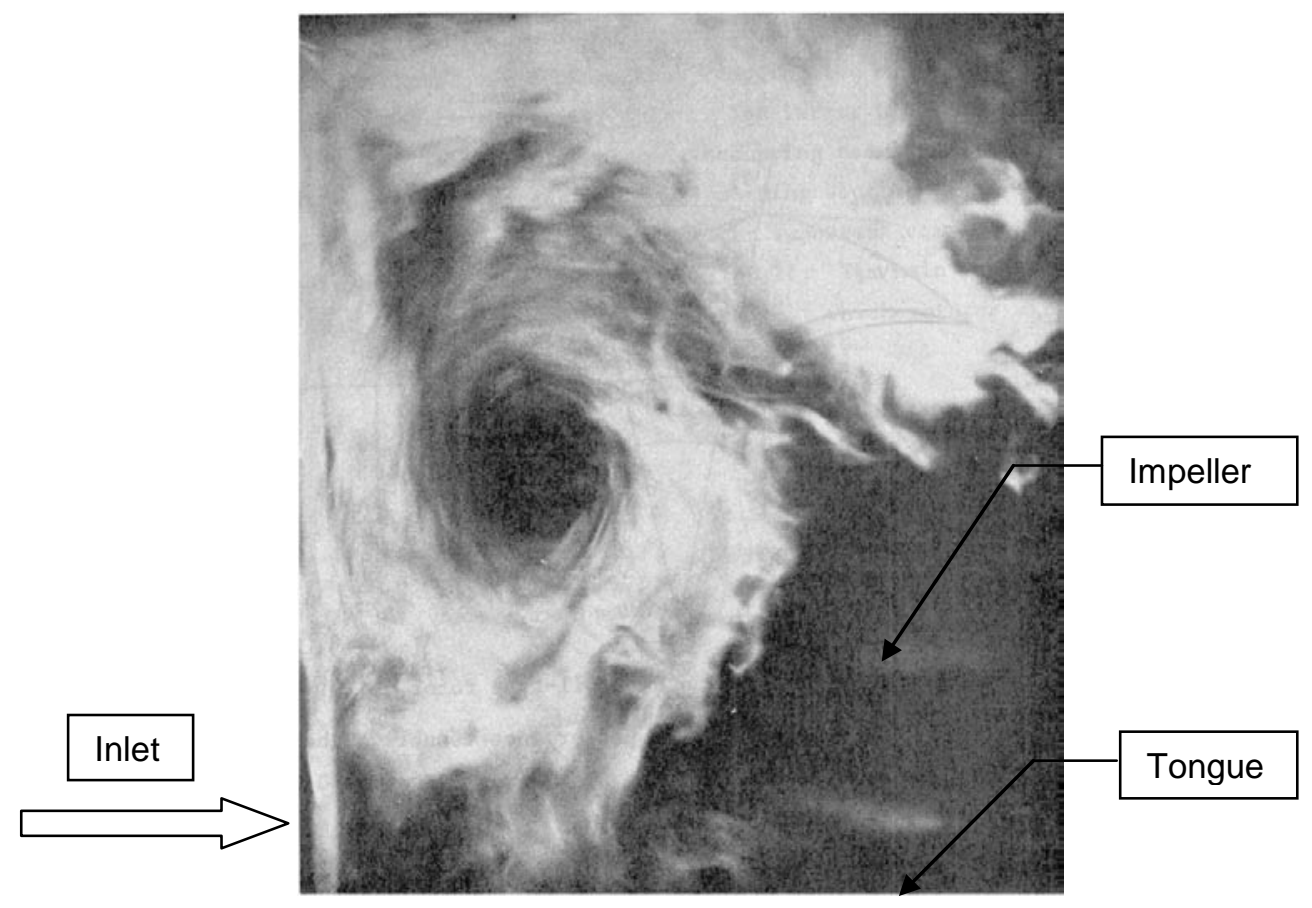

Figure 7. Vortex at the fan outlet. [Mehta, 1977] 


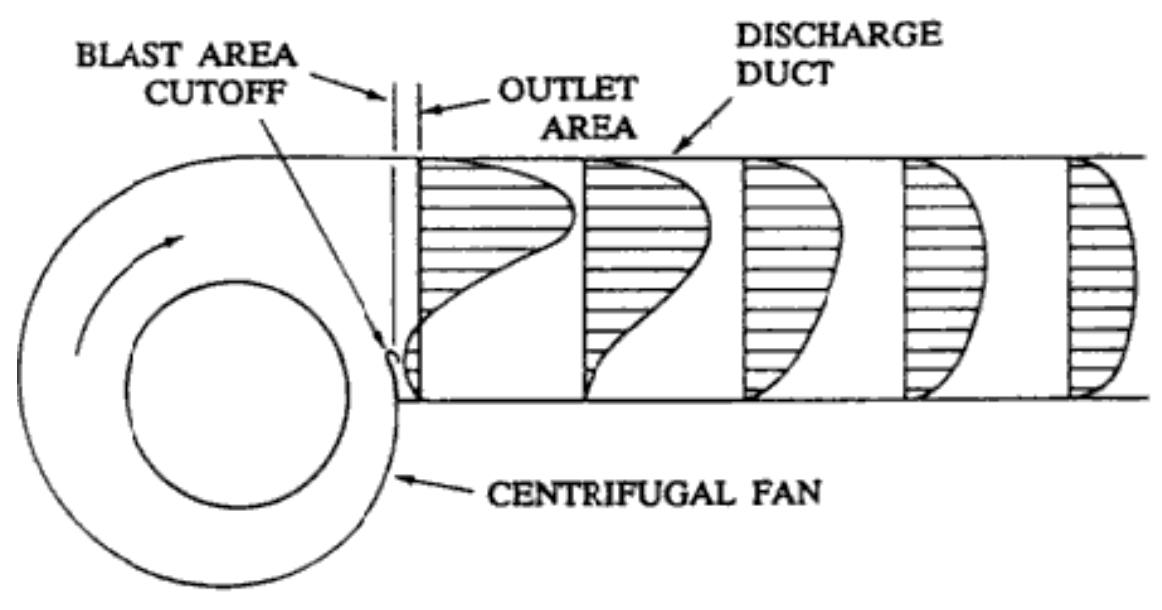

Figure 8. Radial fan exit velocity profile [Mull, 1997]

Mehta (1977) studied the case where a centrifugal fan is connected to a diffuser and it found that the vortex helps to avoid separation in the diffuser (see section 3.4.3.4), nevertheless some flow conditioners (screens and honeycombs) are needed downstream of the diffuser to improve flow uniformity (see section 3.4.4).

Since a fan had already been acquired, and according to Mehta (1977) it has the appropriate characteristics to be used in a wind tunnel, no further analysis about this component will be conducted. The characteristics of the selected fan are listed in section 7.4.

\subsubsection{Wide Angle Diffuser}

The function of the diffuser in a wind tunnel is to connect the fan exit with the settling chamber where a large cross-sectional area and nearly zero velocity (to minimize the pressure losses at the flow conditioners) are required in order to improve the flow steadiness and uniformity. This connection has to be done carefully, to avoid creating flow irregularities; this means that the diffuser has to 
be designed in such way to avoid separation, which due to the adverse pressure gradients is very likely to occur.

To avoid separation in a diffuser, the expansion angle, $2 \theta$ as shown in Figure 9 has to be less than $6^{\circ}$ [Mehta, 1977]. But in some cases, due to the required increment in the area, this leads to very long diffusers. To keep the overall tunnel length manageable a wide angle diffuser is typically used, which are diffusers with a diffuser angle greater than $6^{\circ}$ [Mehta, 1977]. To avoid separation in these diffusers a system of control for the boundary layer has to be provided, such as screens, splitter plates, suction slots, or vanes.

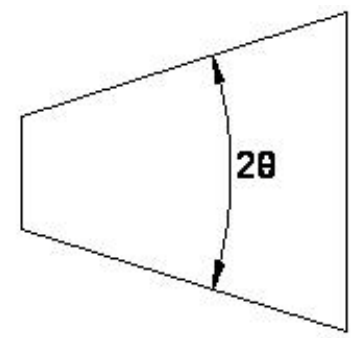

Figure 9. Expansion Angle.

A three-dimensional diffuser has different expansion angles in top and lateral views, in which case $2 \theta$ is defined as the larger one, which will be the critical angle, since separation is more likely to occur at larger angles. Another parameter that identifies a wide angle diffuser is the area ratio, $A$, defined as:

$$
A=\frac{A_{d, e}}{A_{d, i n}},
$$

where, $A_{d, e}$ is the area of the diffuser exit and $A_{d, i n}$ is the area of the diffuser inlet.

Besides the expansion angle and the area ratio, there are other parameters associated to Wide-Angle diffusers. These are described in the following subsections and are related to the boundary layer control method to avoid flow separation. 


\subsubsection{Diffuser Screens.}

Screens are the simplest boundary layer devices used to avoid separation in a wide angle diffuser. Screens are also used as to improve flow uniformity in the settling chamber (see Section 3.4.4.2). Due to its low cost and simplicity of installation they have become a preferred means to improve flow quality in wind tunnels. According to Mehta (1977) screens improve the flow uniformity because they reduce the total pressure of the regions with high velocity more than the regions with low velocity. This also reduces the probability of boundary layer separation.

Mehta (1977) recommends and studies the use of screens as boundary layer control devices, the most important conclusion of that study is provided in Figure 10 [Mehta, 1977], which is the results of a survey where more than 130 wide angle diffusers (studied by several authors) were classified according to their expansion angle, area ratio and their boundary layer control method to avoid separation. Figure 10 [Mehta, 1977] shows only the diffusers that use screens as boundary layer control method and diffusers without any control method. Each mark represents a studied diffuser. This chart also includes unsuccessful configurations (i.e. with separation). Each mark, according to the inset, indicates the wind tunnel cross-sectional shape (rectangular, circular), the number of screens used, the diffuser wall shape (curved or straight), and if the configuration is successful or unsuccessful. The curves identified as 0,1,2 and 3 represents the limits of successful operation with $0,1,2$ and 3 screens respectively. To have a diffuser operating successfully with a determined number of screens, the point in the chart defined by the expansion angle and the area ratio has to be to the left of the curve corresponding to the number of screens used, as an example, if an area ratio of five is needed and two screens are going to be used, the expansion angle has to be approximately between $25^{\circ}$ and $40^{\circ}$. 


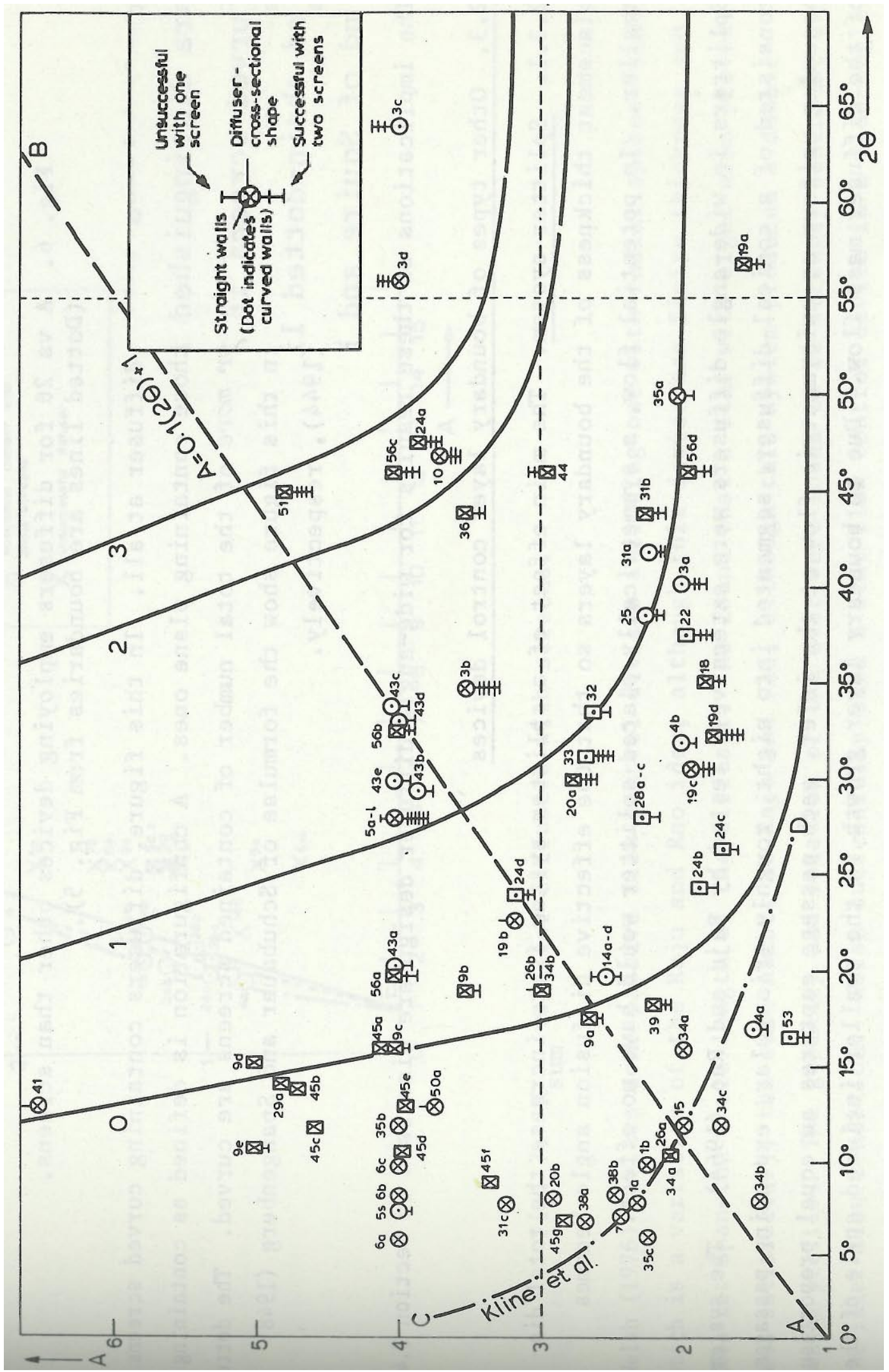

Figure 10. A vs. $2 \Theta$ for several wide angle diffusers [Mehta, 1977]. 
The parameter that relates the geometry of a screen with its influence on the flow is the pressure drop coefficient, $K_{s c r}$, defined as,

$$
K_{s c r}=\frac{\Delta p_{s c r}}{\frac{1}{2} \rho U_{s c r}^{2}},
$$

where, $\Delta p_{s c r}$ is the static pressure drop across the screen, and $\frac{1}{2} \rho U_{s c r}^{2}$ is the dynamic pressure of the flow approaching perpendicularly to the screen. When this parameter is defined the geometry of the screen can be determined (see section 3.4.3.1.3). In order to determine the number of required screens in the diffuser and the values of their $K_{s c r}$ another important conclusion of Mehta (1977) will be used. This conclusion establishes that the summation of the pressure drop coefficients of the diffuser screens, $K_{\text {sum }}$, should be such that:

$$
A<1.12 K_{\text {sum }}+1
$$

Figure 10 [Mehta, 1977] when combined with Eq. 4 can be used as a method for selecting or designing a wide angle diffuser that only uses screens to avoid separation. If the area ratio is known then with Eq. 4 the minimum number of screens can be determined. Then using the chart of Figure 10 [Mehta, 1977] the diffuser angle can be found. This angle is selected taking into account that in order to have a successful operation with the determined number of screens the point corresponding to the diffuser parameters ( $A$ and $2 \theta$ ) must be located to the left of the curve corresponding to the number of screens selected. Mehta (1977) suggests to limit the parameters such that $A<5$ and $2 \theta<50^{\circ}$.

Figure 11 [Mehta, 1985] shows the effect of a screen in the flow across it, the velocity profiles are shown at several distances downstream of a screen; $x$ equal to zero corresponds to the screen location and $x$ equal to $597 \mathrm{~mm}$ is the farthest location where the profile was measured. The velocity profile at $x$ equal to zero without the screen is also shown (labeled Undisturbed in the figure). The 
dimensions of the test section used in the analysis related to Figure 11 were $450 \times 450 \mathrm{~mm}$.

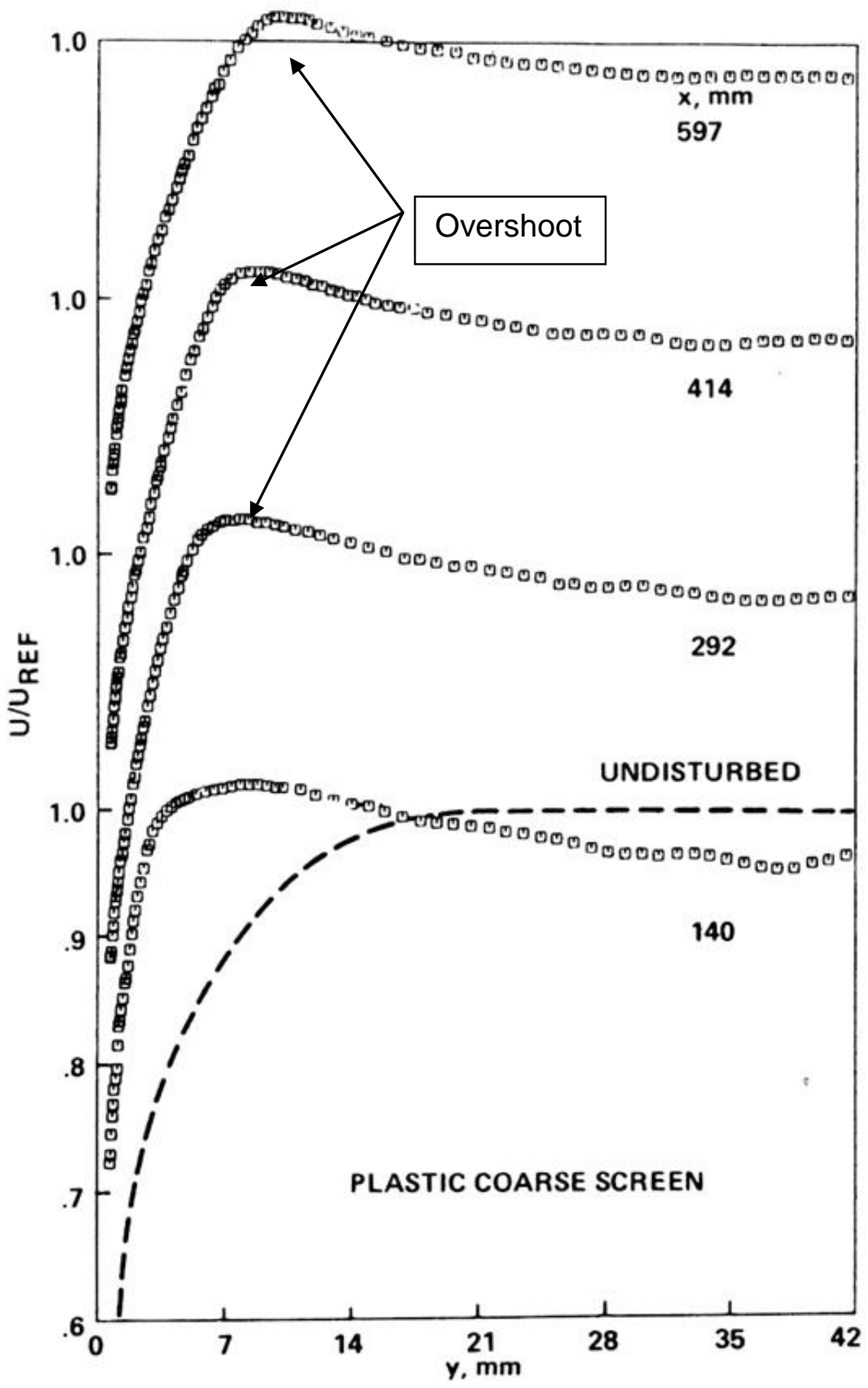

Figure 11. Velocity Profile downstream of a screen [Mehta, 1985] 
According to Mehta $(1977,1985)$ the principal effects of a screen in the flow are:

- Produces a drop in the static pressure proportional to the square of the flow velocity.

- Tends to refract the incoming flow toward the local normal of the screen (except when the incoming flow is normal to the screen), this is illustrated in Figure 12.

- Reduction of the boundary layer thickness.

- A more uniform velocity profile (see Figure 11 [Mehta, 1985]).

- Creates an overshoot in the velocity profile near to the boundary layer (see Figure 11 [Mehta, 1985])

- Changes completely the turbulence distribution and introduces a new boundary layer. The turbulence intensity and scale is reduced.

Screens are easier to install, and construct compared with other methods of boundary layer control (splitter plates, suction slots, vanes, [Mehta, 1977]), and they also have the important advantage that they reduce the turbulence of the flow, which does not occur with the other methods. 


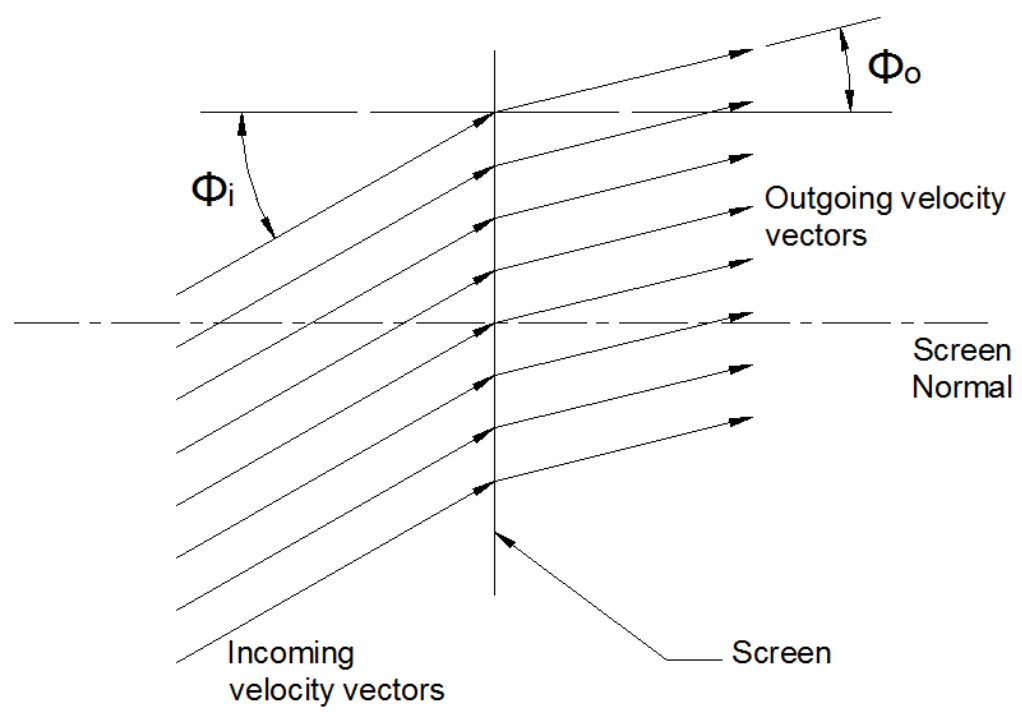

Figure 12. Flow refraction by a screen

The overshoot in velocity is very helpful to overcome adverse pressure gradients, and this explains the use of screens to avoid separation in wide angle diffusers.

\subsection{Screen profile.}

Screens can be curved or flat as is shown in Figure 13. The curved screens have the advantage of increasing the overshoot near the boundary layer, which is helpful in avoiding flow separation. Curved screens are recommended to be used in diffusers so that the screen by itself will not induce separation. As is shown in Figure 13 for a flat screen due to the geometric characteristics, the refraction is outward from the wall, on the contrary, the curved screen avoid separation since it deflects the flow toward the wall. 


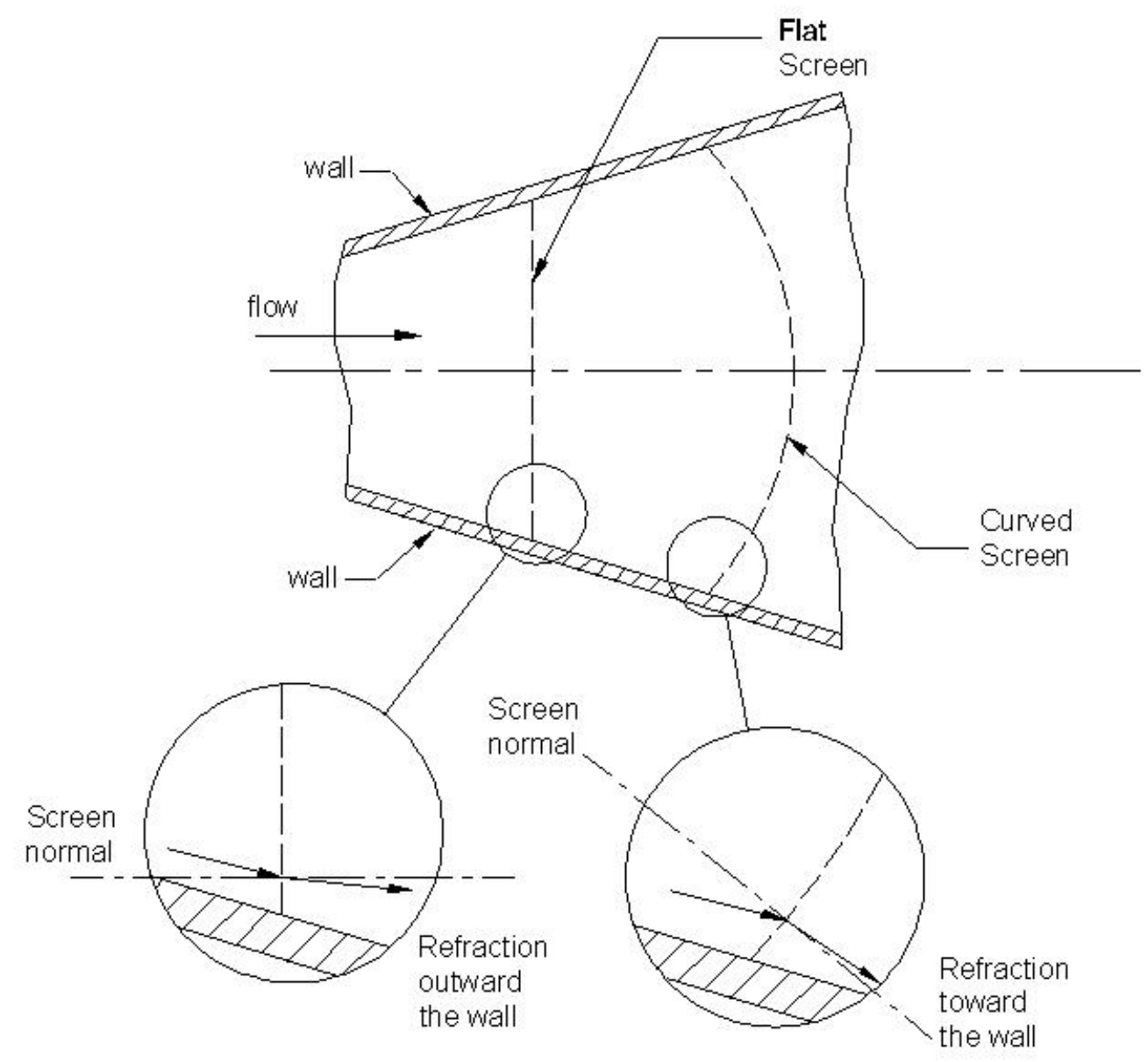

Figure 13. Screen profile and diffraction, curved and flat meshes

\subsection{Screen Material.}

The screens used in wind tunnels are commercially available screens; they usually are either plastic or metallic. The influence of screens in the flow depends also on its material. This is not only due to the mechanical properties of the material but also due to the manufacturing process. The main differences between these screens are described below.

At the nodal points of plastic screens the threads are embedded in each other, so in case of a deflection of the mesh (due to the wind load) the original uniform weave is conserved. In the case of a metal mesh the wires can slide 
over each other and the weave will change depending on the deflection of the screen. As is shown in section 3.4.3.1.3 the screen parameters depend on this weave.

The nodal points of the plastic screens are necked in order to be embedded. In a metal screen wires are superposed this means that the plastic screen is more coplanar.

Due to the material properties plastic screens tend to deflect more with the wind load, allowing them to be curved screens.

Since screens tend to accumulate dust and this can change the flow conditions, it is recommended to use a maximum of three screens (three in the diffuser and three in the settling chamber) and provide an easy access method for cleaning them.

\subsection{Screen Characterization.}

The geometric characteristics of a screen are fully defined by two of the following three factors: wire diameter, $d_{s c r}$, number of opening per unit of length, $1 / l$, and the screen open area ratio $\beta$, defined as:

$$
\beta=\left(1-\frac{d_{s c r}}{l}\right)^{2} .
$$

Recall the effect of a screen in the flow can be characterized by the pressure drop coefficient, $K_{s c r}$, this coefficient is a function of the screen open area ratio, the inlet flow angle and the Reynolds number based on the wire diameter. There are two accepted expressions to find the value of $K_{s c r}$ in terms of $\beta$ for a screen placed normal to the flow, the first one, called Collar's formula, is:

$$
K_{s c r}=\frac{C_{s c r}(1-\beta)}{\beta^{2}},
$$

Eq. 6

which is recommended for velocities greater than $10 \mathrm{~m} / \mathrm{s}$, and $C_{s c r}$ is a coefficient that the depends of the Reynolds number and its value is between 0.9 and 1.0. 
The second expression is recommended for velocities smaller than $10 \mathrm{~m} / \mathrm{s}$, which is called Wieghardt's formula:

$$
K_{s c r}=6.5 \frac{1-\beta}{\beta^{\frac{5}{3}}} R e_{s c r}{ }^{-\frac{1}{3}}
$$

which is a function of the Reynolds number based on the screen diameter $d_{s c r}$. Table 3 [Mehta,1977] shows the values of $K_{s c r}$ for different screens using both equations.

Table 3. Values for the pressure drop coefficient of a screen [Mehta, 1977].

\begin{tabular}{|c|c|c|c|c|}
\hline Mesh & $\begin{array}{c}\text { Wire Diameter } \\
\mathrm{d} \\
{[\mathrm{mm}]}\end{array}$ & $\beta$ & $\begin{array}{c}K \text { accordin to } \\
\text { Collar's formula } \\
\text { (recommended for } \\
U>10 \mathrm{~m} / \mathrm{s} \text { ) }\end{array}$ & $\begin{array}{c}K \text { accordinf to } \\
\text { Wiedhardt's formula } \\
\text { (recommended for } \\
U<10 \mathrm{~m} / \mathrm{s}\end{array}$ \\
\hline 7 & 0.812 & 0.60 & 1.00 & 0.752 \\
\hline 8 & 0.711 & 0.60 & 1.00 & 0.787 \\
\hline 9 & 0.711 & 0.56 & 1.263 & 0.971 \\
\hline 10 & 0.610 & 0.58 & 1.124 & 0.919 \\
\hline 12 & 0.559 & 0.54 & 1.42 & 1.168 \\
\hline 13 & 0.508 & 0.55 & 1.338 & 1.144 \\
\hline 14 & 0.508 & 0.51 & 1.695 & 1.413 \\
\hline 16 & 0.457 & 0.51 & 1.695 & 1.464 \\
\hline 18 & 0.417 & 0.51 & 1.695 & 1.509 \\
\hline 20 & 0.376 & 0.50 & 1.80 & 1.648 \\
\hline 22 & 0.345 & 0.49 & 1.912 & 1.788 \\
\hline 24 & 0.345 & 0.46 & 2.297 & 2.103 \\
\hline 26 & 0.315 & 0.46 & 2.297 & 2.169 \\
\hline 28 & 0.315 & 0.43 & 2.774 & 2.56 \\
\hline
\end{tabular}

Another parameter used to describe the effect of the screen in the flow is the deflection coefficient $\alpha_{s c r}$, which is defined as the ratio between $\phi_{o}$ (emergence angle) and $\phi_{i}$ (incidence angle) from Figure 12,

$$
\alpha_{s c r}=\frac{\phi_{o}}{\phi_{i}}
$$

a semi-empirical relation for this coefficient is given by Mehta (1977): 


$$
\alpha_{s c r}=\frac{1.1}{\left(1+K_{s c r}\right)^{\frac{1}{2}}},
$$

which is valid for screens with $K_{s c r}>0.7$ and it is defined for a plane screen placed normal to the flow axis.

\subsubsection{Screen Location}

Mehta (1977) recommends placing a screen at the diffuser inlet, since at this location the flow is most likely to separate. To determine the position of other screens in the diffuser, Mehta (1977) uses three non dimensional parameters to determine the screen positions $M_{0}, M_{1}, M_{2}$. They are defined as,

$$
\begin{gathered}
M_{0}=\sum_{i=1}^{n} K_{s c r, i}=K_{s u m} \\
M_{1}=\frac{1}{M_{0}} \sum_{i=1}^{n} \frac{x_{i}}{L_{d}} K_{s c r, i}
\end{gathered}
$$

Eq. 11

and,

$$
M_{2}=\frac{1}{M_{0}} \sum_{i=1}^{n}\left(\frac{x_{i}}{L_{d}}-\frac{1}{2}\right)^{2} K_{s c r, i}
$$

where $x_{i}$ is the location (relative to the inlet) of a screen with $K_{s c r, i}, L_{d}$ is the diffuser length and $n$ is the number of screens, which has a maximum of four. If $M_{1}$ tends to zero the screens tend to be at the inlet, and if it goes to one, the screens tend to be located at the outlet of the diffuser. A uniform screen distribution leads to a value of about 0.5 for $M_{1}$. The value of $M_{2}$ is related to the distance between the screens, and its optimum value is related to the diffuser angle. A value of 0.17 for $M_{2}$ is recommended to avoid separation at the inlet 
and outlet corners. The use of fairings at these corners is strongly recommended (see Figure 14) since they help to avoid flow separation.

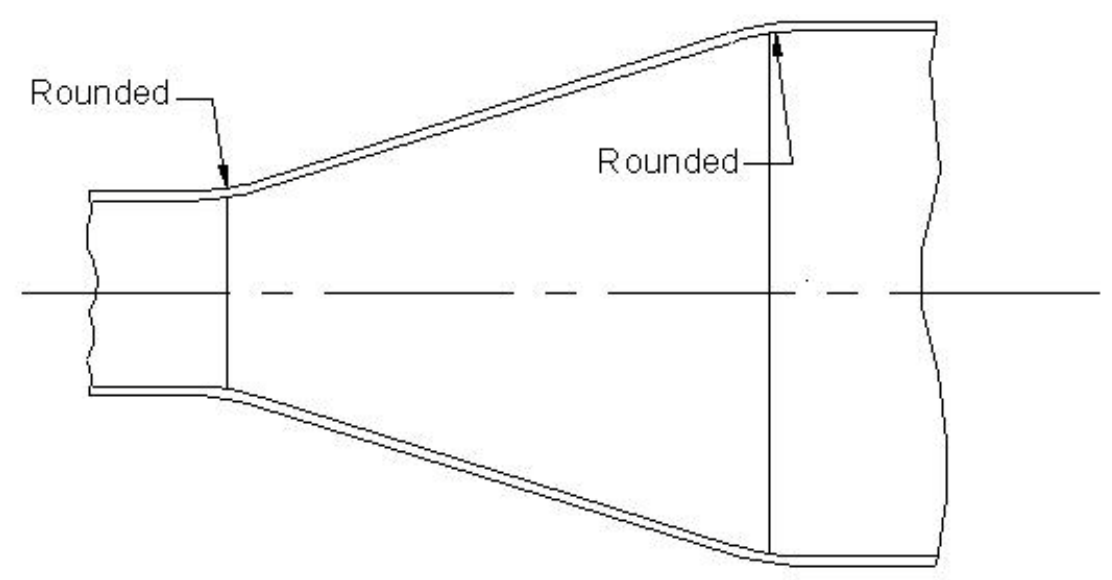

Figure 14. Fairings at the inlet and outlet of a straight wall diffuser

\subsubsection{Diffuser Walls}

The use of curved walls has an advantage in avoiding flow separation, but construction is difficult, for this, straight walls are often used (see Figure 14), and they can work satisfactorily if the diffuser angles are not too large (less than $50^{\circ}$ ).

\subsubsection{Effect of the Fan Vortex}

To find the effect of the fan vortex on the flow in the diffuser, Mehta (1977) studied a centrifugal fan attached to a diffuser with rectangular cross-section, flat walls and an area ratio, $A$, equal to 2.25 and a diffuser angle of $15^{\circ}$. A special feature of this diffuser is that it diverges in two sections separately, i.e. first it diverges in the $Y Z$ plane, and then in the $X Z$ plane, with the flow along the $Z$-axis (see the diagram in the upper part of Figure 15 and Figure 16 [Mehta, 1977]). With this configuration three cases where studied: the first one with no flow conditioners, the second one has a flat screen at the entry of the first portion of the diffuser and a curved screen at the entry of the second portion (each screen with a $K_{s c r}$ of 1.5 , this reduces the swirl angle to about $70 \%$ of its original value [Mehta, 1977]), the last case has a honeycomb at the fan outlet, eliminating the 
vortex almost completely. The result of these studies are shown in Figure 15 and Figure 16 [Mehta, 1977], where the total pressure coefficient, $C_{p}$, was plotted at multiple points at the diffuser exit. This coefficient is defined as,

$$
C_{p}=\frac{p_{t, i}-p_{a t m}}{\frac{1}{2} \rho U_{f, i}^{2}}
$$

where $p_{t, i}$ is the measured total pressure at a specific location, $p_{a t m}$ is the atmospheric pressure, and $U_{f, i}$ is the blower inlet velocity.

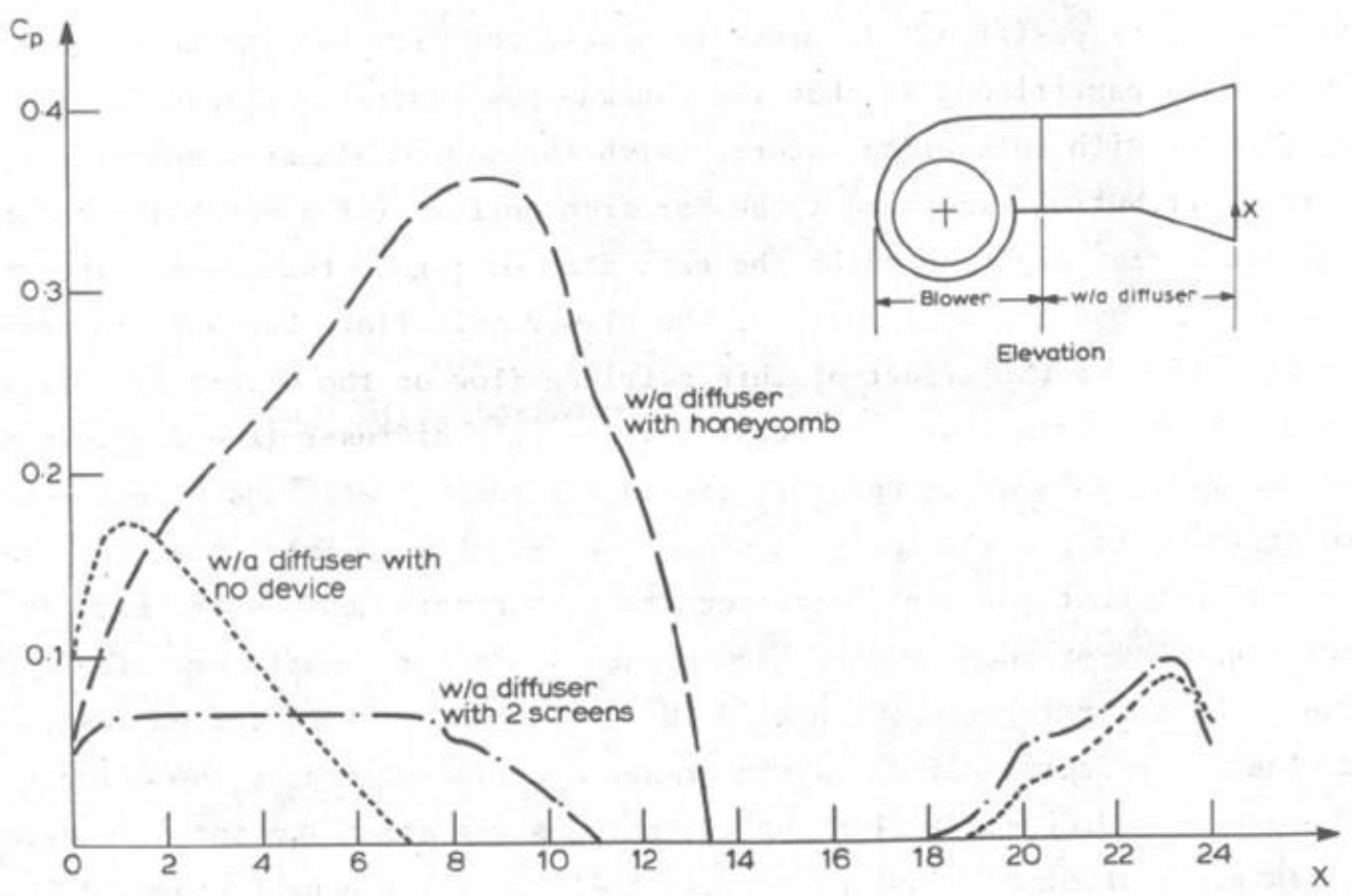

Figure 15. Vertical total pressure distribution at the diffuser exit, for several degrees of swirl [Mehta, 1977]. 


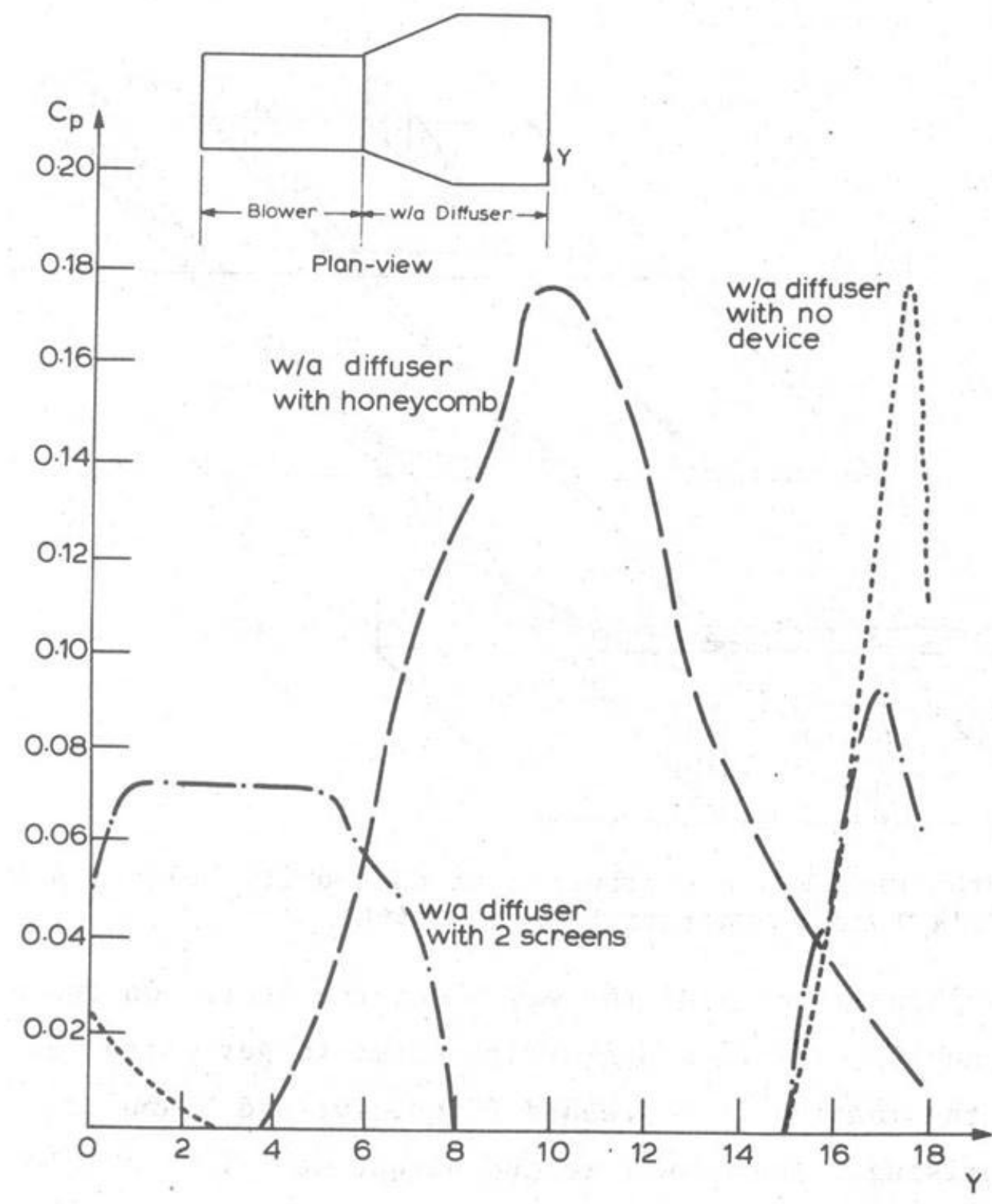

Figure 16. Horizontal total pressure distribution at the diffuser exit, for several degrees of swirl [Mehta, 1977].

As can be seen from Figure 15 and Figure 16 [Mehta, 1977] the vortex can produce an attached flow at the diffuser walls, but with a backflow in the core. The use of two screens reduces the backflow region and improves flow uniformity. The honeycomb removes the vortex almost completely. But the flow is completely separated at the wall adjacent to the fan cutoff (or tongue). This shows that the vortex helps to avoid separation in the diffuser walls, thus it is not 
recommended to install honeycombs at the fan outlet, when using wide angle diffusers.

\subsubsection{Settling Chamber}

The settling chamber is the region in a wind tunnel where the flow uniformity is improved and the turbulence is reduced. This is done by using flow conditioners: honeycombs and screens. The purpose of the settling chamber is to create a segment of the wind tunnel with a low speed and large area. The low speed is required to position the flow conditioners since this will reduce the energy losses, which are proportional to the velocity squared. The large area is required to permit the contraction to be placed in the wind tunnel for further improve flow uniformity. A settling chamber can be considered a component where the characteristics of the flow (velocity profiles and turbulence) that comes from the fan are "erased" and more convenient ones are imposed. The main characteristics of the flow conditioners are discussed in this section.

\subsubsection{Honeycombs.}

Honeycombs are used to make the flow direction more parallel to the wind tunnel walls, they remove swirl and correct the direction of the incoming flow, which has these undesirable characteristics originated by the fan and the diffuser.

Honeycombs are recommended when the yaw angles of the flow are smaller than 10 degrees, if larger yaw angles are expected they can be reduced by using a screen upstream of the honeycomb, for example a screen with a $\mathrm{K}$ equal to 1.5 reduces yaw angles by a factor of 0.7 [Mehta, 1977]. The honeycombs are defined by the size and shape of the cells, usually with hexagonal shapes, but for ease of construction, square or triangular shapes can be used. The recommended length to diameter ratio of the cells is between 7 and 10, and for small low speed wind tunnels, cell diameters of about $5 \mathrm{~mm}$ are frequently used [Mehta, 1977], which leads to a maximum cell length of $50 \mathrm{~mm}$. 


\subsubsection{Settling Chamber Screens.}

As was mentioned in section Error! Reference source not found. creens are placed in the settling chamber in order to reduce the turbulence, and improve flow uniformity. Small low speed wind tunnels, with wide angle diffusers, usually use three screens with a $K_{s c r}$ equal to approximately 1.5 .

\subsubsection{Flow conditioners arrangement.}

Usually the honeycomb is placed downstream of the diffuser exit, and the screens are placed downstream of the honeycomb, except when a screen upstream of the honeycomb is required due to severe yaw angles.

The distance between the screens is recommended to be equal to about 500 wire diameters [Mehta, 1977]. This guarantees that the wakes of the wires of one screen diminishes before the next downstream screen is reached.

The optimum distance between the last screen and the contraction inlet is about 0.2 cross-sectional diameters [Mehta, 1977] if this distance is much longer there will be an excessive boundary layer growth. On the contrary if it much shorter the disturbance created by the last screen could reach the contraction inlet. In both cases this will have an undesirable effect in the flow uniformity of the test section.

\subsubsection{Contractions}

Contractions are located after the settling chamber to increase the flow velocity, and to increase the uniformity of the longitudinal mean velocity. The most important parameter when studying contraction is the contraction ratio $\boldsymbol{c}$, which is defined as the ratio of the inlet area and the outlet area of the contraction

The effect of a contraction in reducing axial velocity variations can be explained following the procedure expressed by Bradshaw (1964) using the diagram presented on Figure 17 [Bradshaw, 1964], where it is assumed that the 
contraction inlet has a small region with a higher velocity (i.e. a non-uniformity) equal to $U_{c, i}$ times $\delta$, where $U_{c, i}$ is the main stream velocity (assumed constant) and $\delta$ is the velocity variation factor. The total pressure of the main stream at the inlet, $p_{t, c, i, l}$, is,

$$
p_{t, c, i, l}=p_{c, i}+\frac{1}{2} \rho U_{c, i}^{2} .
$$

From Figure 17 [Bradshaw, 1964] it can be seen that at the inlet the total pressure in the high velocity region, $p_{t, c, i, h}$, is,

$$
p_{t, c, i, h}=p_{c, i}+\frac{1}{2} \rho U_{c, i}^{2}(1+\delta)^{2},
$$

where it is assumed that the static pressure is constant across the inlet. The velocity of the main stream at the outlet, $U_{c, e}$, becomes approximately,

$$
U_{c, e}=c U_{c, i}
$$

here the extra mass flow in the high velocity region is neglected. Applying Bernoulli's equation to the main stream between the inlet and the exit, yields,

$$
p_{c, i}+\frac{1}{2} \rho U_{c, i}^{2}=p_{c, e}+\frac{1}{2} \rho c^{2} U_{c, i}^{2},
$$

solving for the static pressure at the exit, $p_{c, e}$,

$$
p_{c, e}=p_{c, i}+\frac{1}{2} \rho U_{c, i}^{2}\left(1-c^{2}\right),
$$

applying Bernoulli's equation to the high velocity region,

$$
p_{c, i}+\frac{1}{2} \rho U_{c, i}^{2}(1+\delta)^{2}=p_{c, e}+\frac{1}{2} \rho U_{c, e, h}^{2}
$$


where $U_{c, e, h}$ is the velocity of the high velocity region at the exit. Using the value for $p_{c, e}$ from Eq. 18, and solving for $U_{c, e, h}$,

$$
U_{c, e, h}=\left(1+\frac{2 \delta+\delta^{2}}{c^{2}}\right)^{\frac{1}{2}} c U_{c, i}
$$

and using a Taylor's series approximation, provides,

$$
U_{c, e, h}=\left(1+\frac{2 \delta+\delta^{2}}{2 c^{2}}\right) c U_{c, i}
$$

Eq. 21

Assuming $\delta<1$ (velocity variations are smaller than the main stream velocity), and $\delta^{2} \ll 2 \delta$, then,

$$
U_{c, e, h}=\left(1+\frac{\delta}{c^{2}}\right) c U_{c, i}
$$

From the diagram the high velocity at the inlet is,

$$
U_{c, i, h}=(1+\delta) U_{c, i}
$$

By definition the relative velocity variation at the inlet is,

$$
\delta_{c, i}=\frac{U_{c, i, h}-U_{c, i}}{U_{c, i}}
$$

Inserting Eq. 23 into Eq. 24, yields,

$$
\delta_{c, i}=\delta
$$

and at the exit it is,

$$
\delta_{c, e}=\frac{U_{c, e, h}-U_{c, e}}{U_{c, e}} .
$$


Substituting Eq. 22 and Eq. 16 into Eq. 26 and simplifying, yields,

$$
\delta_{c, e}=\frac{\delta_{c, i}}{c^{2}},
$$

as can be seen from this relationship the relative velocity variation is reduced by a factor of $c^{2}$. For low speed wind tunnels the acceptable maximum exit nonuniformity is about $\pm 0.5 \%$ velocity variation outside of the boundary layers [Mehta, et al., 1979].

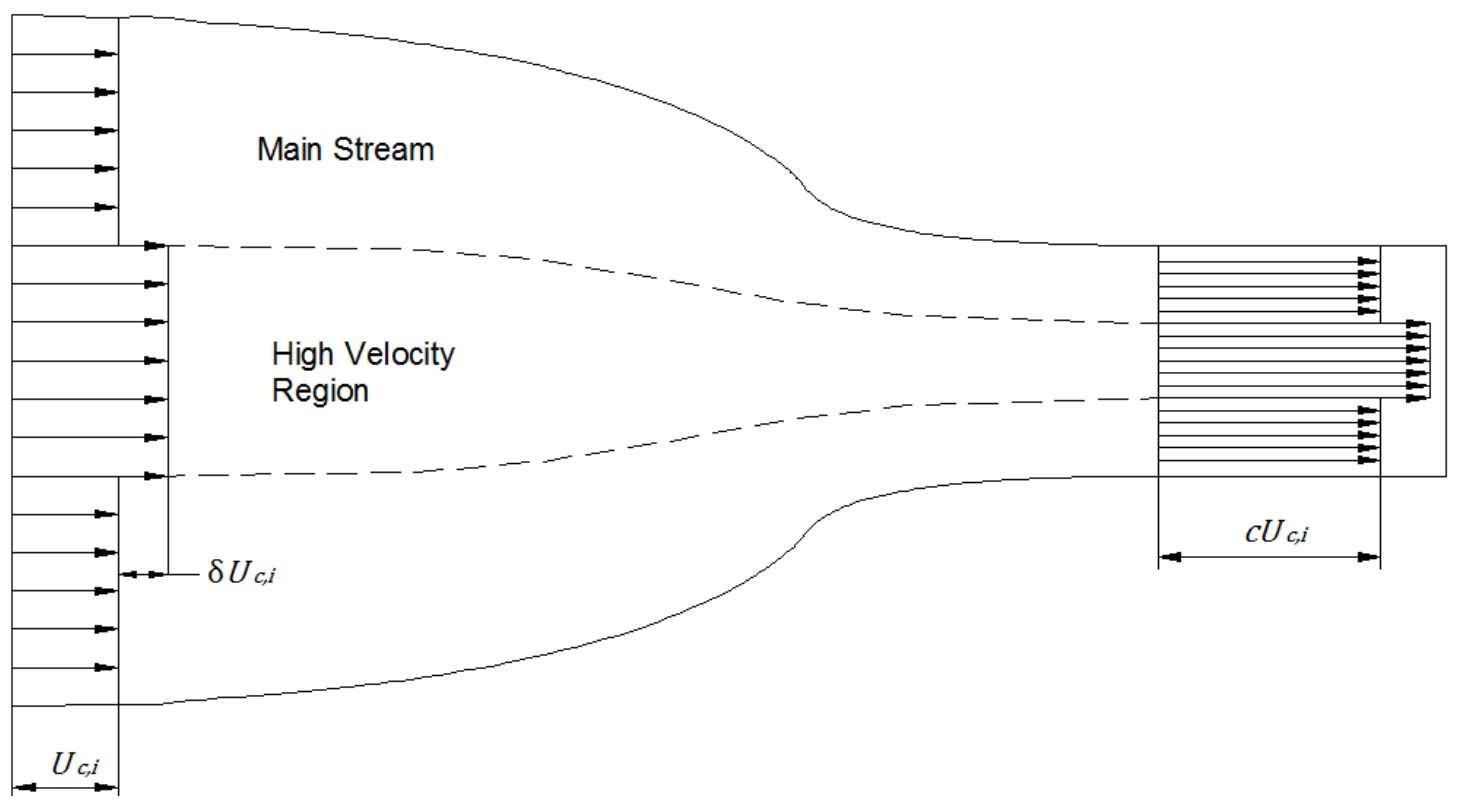

Figure 17. Variation in the Axial velocity non-uniformities in a contraction [Bradshaw, 1964]

The contraction also affects the transverse mean velocities, but in this case the variations are increased. Regarding the fluctuating velocities, the variations in the longitudinal fluctuating velocity, $u^{\prime}$, is reduced while the variations in the transverse components, $\boldsymbol{v}^{\prime}$ and $\boldsymbol{w}^{\prime}$, are increased [Mehta, 1977]

Although a contraction is installed in a wind tunnel to improve the flow quality (uniformity and steadiness) at the exit, an incorrectly designed contraction can, on the contrary, deteriorate flow quality. A well designed contraction minimizes the boundary layer growth, this leads to a reduction in the contraction 
length, but as the contraction length decreases stronger adverse pressure gradients appear near the walls of the contraction ends, especially at the inlet, where the situation is worsened due to the "destabilizing" effect of the concave curvature on the boundary layer [Bell, et al., 1988], [Bell, et al., 1989]. In the worst case this can lead to flow separation, decreasing the flow quality at the test section. Therefore the flow conditions in the contraction have to be studied in order to select the contraction length.

At first sight it does not seem reasonable that adverse pressure gradients can occur in the contraction, since the flow velocity is increased as a result of the area reduction, nevertheless Bell, et al. (1988) show that using the transverse velocity component of the Navier Stokes equation yields,

$$
\frac{\partial p}{\partial y} \cong-\rho U \frac{\partial V}{\partial x} \cong \frac{\rho U^{2}}{R},
$$

where $V$ is the transverse velocity, $R$ is the local radius of curvature of the streamline near the wall, $x$ is the longitudinal coordinate and $y$ a transversal component (see Figure 18). According to this equation, at a given section the pressure increases from the center to the wall, where it reaches its maximum at the wall, and since it is a maximum value, it is larger than the average pressure at that section.

Now, considering a section of the contraction near to the inlet, it is possible that the maximum value at the wall can be greater than the wall pressure of a point upstream of the inlet section. This means that there is an adverse pressure gradient in the contraction. Downstream of this section, the pressure reduction due to the strong area reduction overcomes this transversal variation effect, and the adverse pressure gradient disappears. The adverse pressure gradient reappears at the exit, but in this case the situation is less critical since the boundary layer thickness has been reduced due to the favorable pressure gradients in the contraction, and a thinner boundary layer is less likely 
to separate. The convex curvature of the contraction wall also helps to avoid flow separation [Bell, et al., 1988], [Bell, et al., 1989].

At the corners of a contraction with rectangular cross-section there are very low velocities and therefore this is a region where separation is more likely to occur, due to this it is recommended to fill the corners. Another phenomenon that affects the flow quality at the test section is cross flow. Cross flow occurs in non-symmetric contractions, such as rectangular cross-sections. This tends to increase the boundary layer thickness [Downie, et al., 1984]. Although these phenomena decrease the flow quality in the test section, a flow with an acceptable quality can be obtained using a contraction with rectangular crosssection by choosing an adequate wall shape.

In addition to the contraction ratio, there are other geometric variables that are important in the study of contractions, which are described in this subsection.

\subsubsection{Contraction Ratio}

For small low speed wind tunnels it is recommended to use contraction ratios between six and nine. The larger values in this range are recommended in order to minimize the energy losses in the honeycombs and screens in the settling chamber.

\subsubsection{Contraction Length}

The contraction length is a parameter that is usually minimized when designing a contraction and its value is chosen to avoid flow separation, which is less likely to occur as the contraction length increases. But if this length is more than the required length, unnecessary boundary layer growth will occur, thus an optimum value can be determined.

\subsubsection{N-Dimensionality}

A contraction can be two-dimensional or three-dimensional. In a twodimensional contraction the inlet and exit have the same height (or width), and in 
a three-dimensional contraction these variables have different values at the inlet and exit.

\subsubsection{Contraction aspect ratios}

The aspect ratio at the contraction inlet and exit is defined as the ratio of the width to the height at the corresponding station. In three dimensional contraction it is often recommended to have the same aspect ratios at the inlet and at the exit to avoid flow distortion [Downie, et al., 1984], [Gibbings, 1993] but usually when designing a wind tunnel as a whole, in order to reduce the angle of the diffuser, this can not be achieved, and different aspect ratios are used [Gibbings, 1993], [Downie, et al., 1984]. Nevertheless, Su (1991) reports that flow uniformity can be slightly improved using different aspect ratios.

\subsubsection{Contraction Wall shape}

There are several methods proposed to find an adequate shape for the contraction walls [Bell, et al., 1988], [Su, 1991], [Downie, et al., 1984], [Gibbings, 1993]. The walls are designed in order to avoid separation, minimize the boundary layer growing, and have a flow with the required uniformity at the exit.

\subsubsection{Contraction Design}

In the design of a contraction the inlet section is usually known (due to the requirements of the fan and diffuser) and some information about the exit is known, for example the aspect ratio, or the required uniformity of the flow. The design of the contraction consists basically in finding the contraction ratio, the length and the wall shape.

There are several methods for designing contractions [Downie, et al., 1984], [Bell, et al., 1988], [Su, 1991], [Gibbings, 1993] some of them use analytical approaches that assume inviscid flow, and then applied some corrections to take into account the effects of the boundary layers. Su (1991) and Downie, et al. (1984) propose some arbitrary shapes based on a family of curves 
(Su (1991) polynomials and Downie, et al. (1984) ellipses), and by modeling the flow over them (computational or experimental) to find several recommendations in order to have a successful contraction. Another method, called design "by eye" is describe by Mehta (1977) and is a method based on experimental data of successful contractions.

All of these methods only give recommendations about the contractions parameters, and they do not give specific design information based on the initial information of the contraction. Due to this, several geometries need to be evaluated and then optimized according to the specific requirements. This optimization requires the modeling of the flow in the contraction, and this can be done experimentally or using numerical analysis as was done by Su (1991). The method that will be used in the present work is the one proposed by Su (1991), which is a straightforward method that uses a two polynomial of different order for the wall shape. Using a computational modeling technique, it gives certain recommendations for several non-dimensional parameters related to the geometric variables described in the above sections. The polynomial proposed by Su (1991) for the contraction height, $H_{c}$, is;

$$
H_{c}(x)=\left\{\begin{array}{ll}
\frac{H_{c, e}-H_{c, i}}{X_{p}^{n_{1}-1}}\left(\frac{x}{L_{C}}\right)^{n_{1}}+H_{c, i}, & 0 \leq \frac{x}{L_{C}} \leq X_{p} \\
\frac{H_{c, i}-H_{c, e}}{\left(1-X_{p}\right)^{n_{2}-1}}\left(1-\frac{x}{L_{C}}\right)^{n_{2}}+H_{c, e}, & X_{p}<\frac{x}{L_{C}} \leq 1
\end{array},\right.
$$


and for the contraction width,

$$
W_{c}(x)=\left\{\begin{array}{ll}
\frac{W_{c, e}-W_{c, i}}{X_{p}^{n_{1}-1}}\left(\frac{x}{L_{C}}\right)^{n_{1}}+W_{c, i}, & 0 \leq \frac{x}{L_{C}} \leq X_{p} \\
\frac{W_{c, i}-W_{c, e}}{\left(1-X_{p}\right)^{n_{2}-1}}\left(1-\frac{x}{L_{C}}\right)^{n_{2}}+W_{c, e}, & X_{p}<\frac{x}{L_{C}} \leq 1
\end{array} .\right.
$$

The variables in these equations are related to Figure 18 and are described below:

$x$ : $\quad$ Distance from the contraction inlet along the contraction longitudinal axis.

$H_{c}(x)$ : Height of the contraction at location $x$

$H_{c, i}$ : Height of the contraction at the inlet.

$H_{c, e}$ : Height of the contraction at the outlet.

$W_{c}(x)$ : Width of the contraction at location $x$

$W_{c, i}:$ Width of the contraction at the inlet.

$W_{c, e}$ : Width of the contraction at the outlet.

$L_{C}$ : Contraction length.

$n_{1}$ : Order of the polynomial of the first portion of the contraction.

$n_{2}$ : Order of the polynomial of the final portion of the contraction.

$X_{p}$ : Location of the points where the two polynomials overlap. 


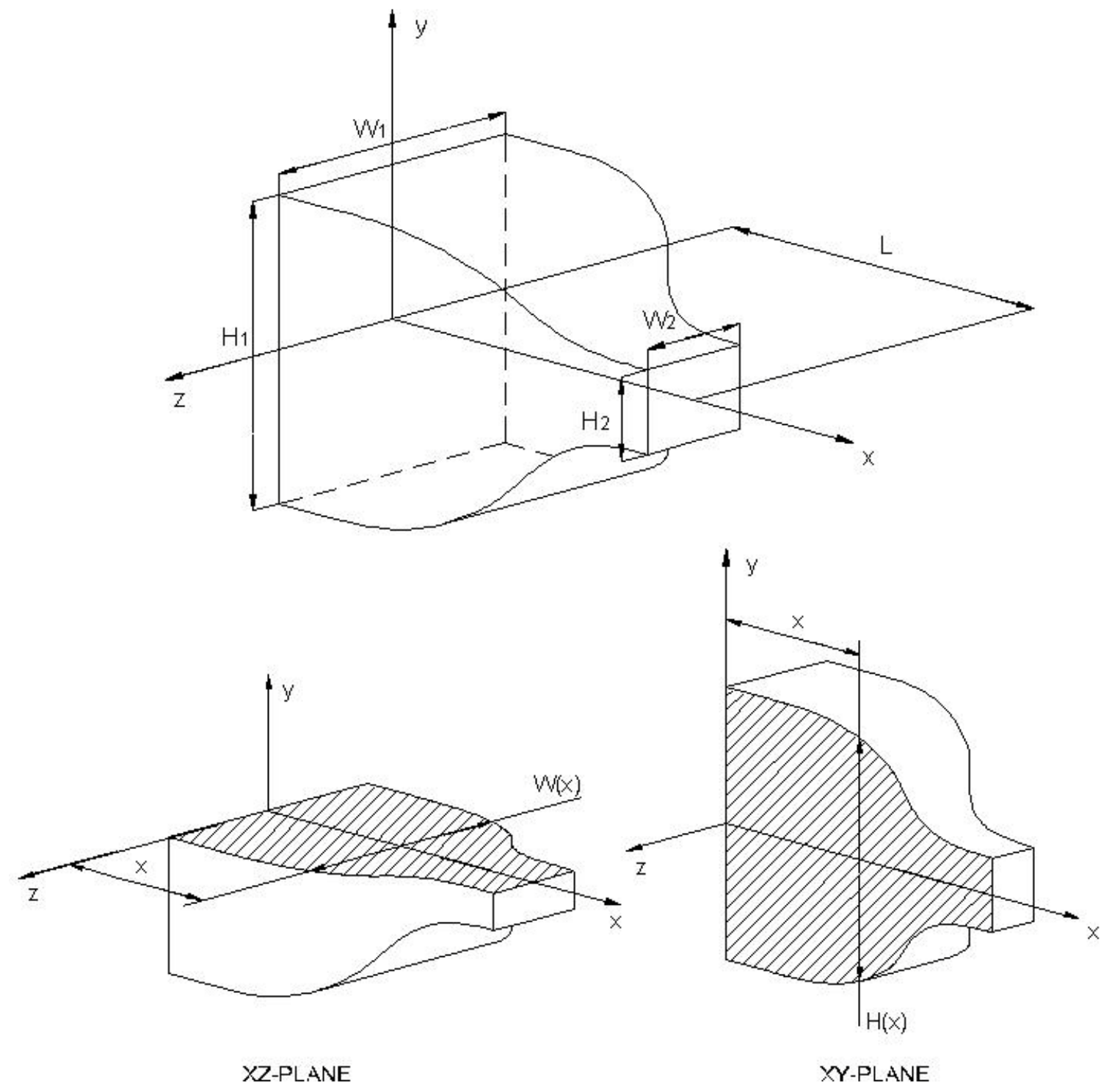

Figure 18.Contraction geometry

The following are the contraction parameters studied by Su (1991) and the recommended values:

$L_{C} / D_{c, i}$ : Where $D_{c, i}$ is the square root of the inlet area. According to Su (1991) as this parameter increases the pressure gradients, uniformity, and cross-flow features are improved, but there is neither an optimum value nor a limit for this parameter. 
$X_{p}$ : This is called the shape parameter and is the point where the two polynomials that describe the wall shape overlaps. As this value increases the flow uniformity at the contraction exit decreases, but it has no effect on the cross-flow. The value for this parameter is chosen by an optimization process.

$c$ : As this parameter is increased the uniformity of the flow at the contraction exit improves.

$A R_{c, i}:$ This is the aspect ratio of the contraction inlet. The recommended value for this parameter is one in order to reduce the pressure gradients and the non-uniformities.

$A R_{c, e}$ : This is the aspect ratio of the contraction exit. There is no recommended values for this parameter, nevertheless if $A R_{1 C}$ is equal to one, as $A R_{2 C}$ increases the pressure gradients and non-uniformities are reduced slightly

$n_{1}$ : This is the order of the polynomial for the first part of the contraction, the recommended values is three.

$n_{2}$ : This is the order of the polynomial for the last part of the contraction, the recommended values is seven.

\subsection{WIND TUNNEL CORRECTION FACTORS}

In a wind tunnel the walls of the test section affects the flow over the model, potentially making the testing conditions different than the real flight conditions. Between these effects are those that are neither due to the viscous boundary layers, or the irregularities in the flow (swirl, nor uniformities, normal velocity, 
etc), these are due to the presence of the walls that impede the air flow from expanding freely, as in real conditions. The data obtained from the wind tunnel tests have to be corrected in order to scale them properly according to the nondimensional parameter (Reynolds number, Froude number, etc) that are relevant to the problem in the study. There are also recommendations for making these effects negligible. In this case, the bottom wall, floor, is also present in the real flight conditions and thus its effect does not have to be corrected for the Air-Ray prototype.

This section discusses the effects of the walls that have an important influence for the present case.

\subsubsection{Solid and Wake Blockage.}

Due to the presence of the model in the test section the flow area is reduced and the air velocity increases. This increases the dynamic pressure and all of the forces and moments experienced by the model. This effect is negligible in open test sections where the air can expand as in the real conditions.

In the test section the model wake is not free to expand due the wall restrictions, the effect of the wall increases as the wake increases. For closed test sections this leads to a increase in the drag. This effect is usually negligible for an open test section, since the air can expand freely.

To minimize the calculations of finding the correction factors for the Solid and Wake Blockage, Barlow, et al., (1999) recommend a maximum value of $7.5 \%$ for the ratio of model frontal area to test section cross-sectional area (blockage area).

\subsubsection{Alteration of the local angle of attack along the span}

If the wing tips are close to the lateral walls of the wind tunnel, the angles of attack are increased excessively, and for an open test section the effects are 
the opposite. For both cases this effect can be negligible if the model span is less than 0.8 of the width of the test section.

\subsubsection{Buoyancy}

The pressure drop along the test section, due to the viscous effects, creates an additional drag force, but usually it is small and can be neglected. There are other effects that have to be corrected in a wind tunnel, mainly the ones related to the effects of the bottom and top walls. In this current work these factors are not considered since the bottom wall is a real condition (it represents the ground), see section 3.6.

The methods for correcting the above effects are not treated here, but they can be found in [Barlow, et al., 1999] and [Heyson, 1971]. Heyson (1970), Recant (1939) and Brown (1939) conducted tests of aircraft in ground effect and affirm that the calculated correction factor where small enough to be neglected. Chawla, et al. (1990) tested a wing in ground effect and uses a blockage area of $7 \%$ and no corrections factors.

\subsection{GROUND SIMULATION}

To simulate the flying conditions of the Air-Ray appropriately, the fact that the aircraft is flying very close to the ground has to be considered carefully. When the prototype craft moves in still air there is a relative velocity between the vehicle and the surface, but since there is no relative movement between the air and the ground there is no boundary layer between the ground and the air, as can be seen in Figure 19a [Turner, 1966] (nevertheless there is a boundary layer between the vehicle and the air). When studying the vehicle in the wind tunnel this condition has to be present in the simulation. If the simulation is carried in a regular wind tunnel, there will be a boundary layer between the tunnel floor and the airstream, and as can be seen in Figure 19b [Turner, 1966] this will change the aerodynamics to which the model is exposed. 


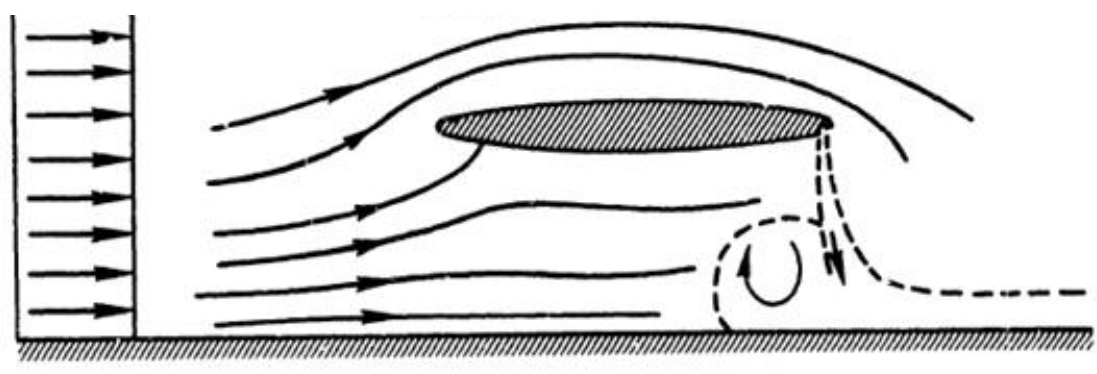

(a) Moving model or moving ground plane simulation

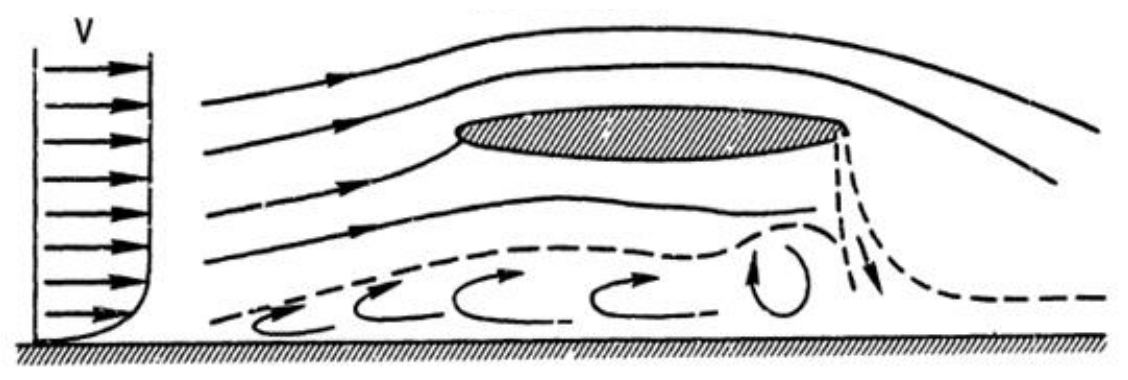

(b) Fixed model or fixed ground plane simulation

Figure 19. Differences between moving model or moving ground plane simulation and fixed model or fixed ground plane simulation [Turner, 1966]

To simulate the actual flight conditions the ground boundary layer has to be eliminated, this can be done by several methods, the two most common are the floor tangential-blowing and the moving belt. In the tangential blowing technique, compressed air is injected into the boundary, using jets located along the floor, this technique is used for high airstream velocities (which is not the case of the present study). In the moving belt technique, the floor is exchanged for a belt that moves at the same speed as the airstream. This one is the most exact technique for simulating the ground [Turner, 1966]. Figure 20a [Turner, 1966] shows when the use of a moving belt is required. In this figure $h$ is the distance from the ground to the model and $b$ is the span of the vehicle, $C_{L}$ is the value of the lift coefficient where the lift coefficient vs. angle of attack, $\alpha$, curve 
using a belt (moving at the same air velocity) diverges from the curve of lift coefficient vs. angle of attack with a fixed ground (Figure 20b [Turner, 1966]).

- DOUBLE SLOTTED FLAF, $A=10$

$\square$ JET FLAP, $A=6$

$\Leftrightarrow$ TILT WING, $A=8.5$

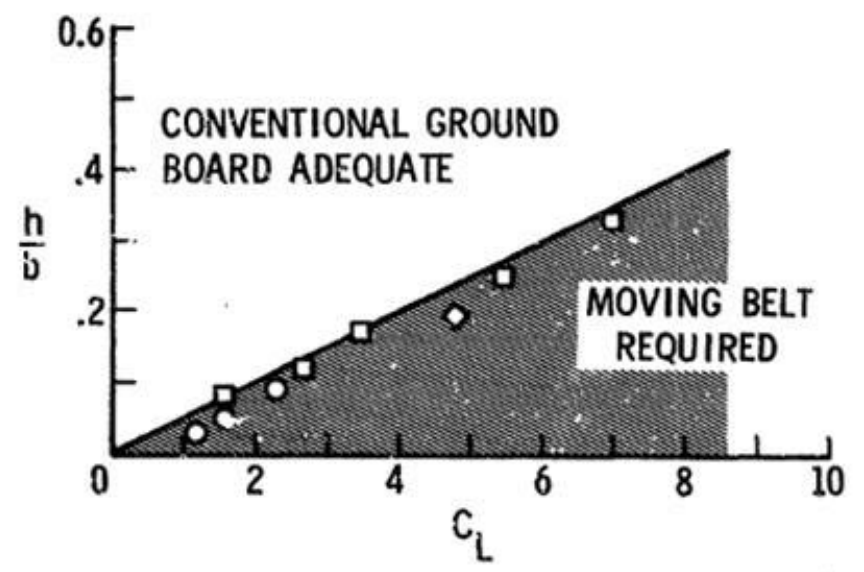

(a)

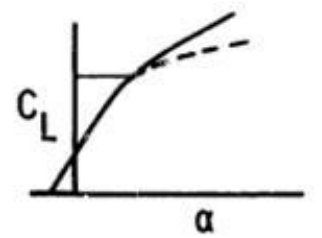

(b)

Figure 20. Selection of the ground simulation technique [Turner, 1966]

Figure 21 [Turner, 1966] shows the variation of the lift coefficient in ground effect with the variation of the belt velocity, this is not shown directly but with the use of non-dimensional parameters. In this figure $\Delta L_{h}$ is the lift loss in ground effect, and is equal to,

$$
\Delta L_{h}=L_{h}-L_{h=\infty},
$$

where $L_{h}$ is the lift at height $h$, and $L_{h=\infty}$ is the lift out of ground effect, $C_{L, h=\infty}$ is the lift coefficient out of ground effect, which has a value of 7.4 for the data of Figure 21 [Turner, 1966]. $V_{B E L T}$ is the belt linear velocity and $V_{A I R}$ is the air velocity. From this figure it can be seen that a small variation between the belt velocity and air velocity is not critical for the measurements in the lift force. 


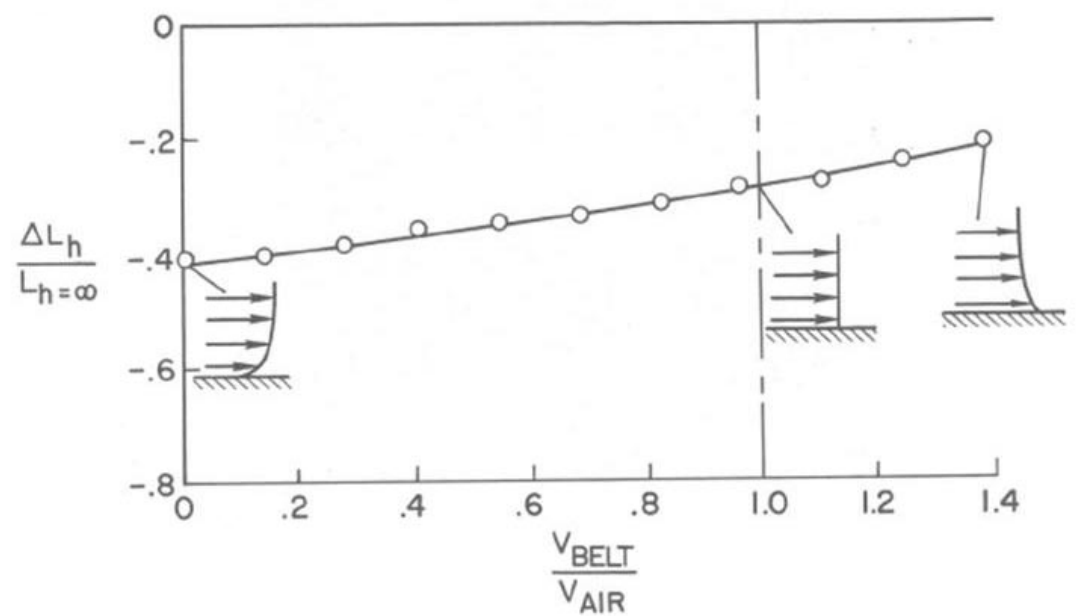

Figure 21. Influence of the belt velocity, $\mathrm{h} / \mathrm{b}=0.166, C_{L, h=\infty}=7.4 \quad[$ Turner, 1966]

The air that comes from the contraction has a boundary layer. In order to have a flow similar to the real conditions, it is helpful to remove this boundary layer before it reaches the belt. This can be done by extracting it at the beginning of the belt, as is shown in Figure 22 [Turner, 1967], which uses a slot located upstream of the moving belt.

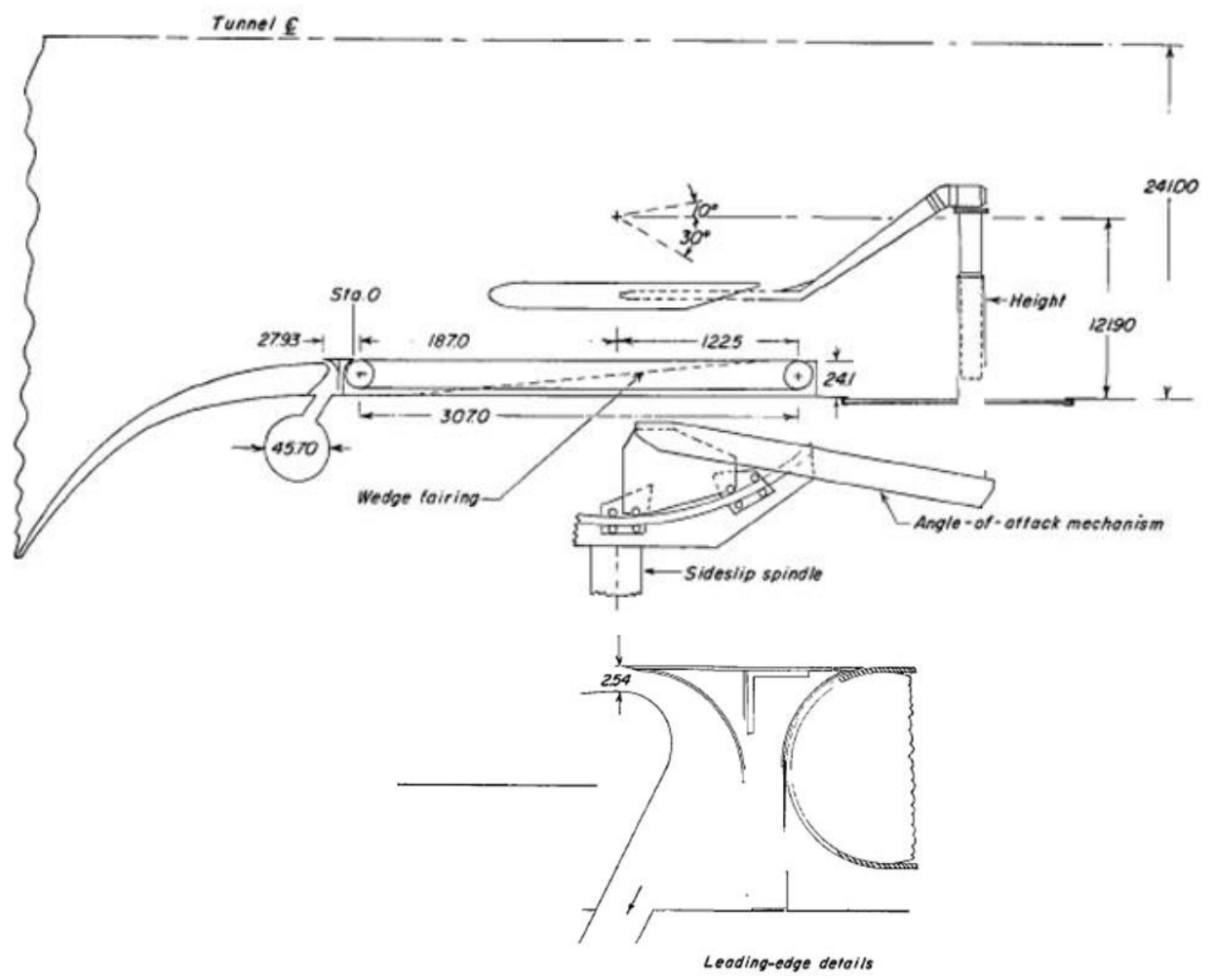

Figure 22. Boundary layer sucking at the belt [Turner, 1967] 
The belt should extend least "one tail length ahead of model nose and one tail length behind the model tail" [Pope, et al. 1966]. A tail length is defined as the distance from airplane center of gravity to aerodynamic center of the horizontal tail. Since the Air-Ray has no horizontal tail the belt length will be assumed as three times the model length, which is slightly larger than the recommended value. The belt has to be supported carefully to avoid excessive vibrations.

\subsection{COMPARISON CASES}

Table 4 shows some data about the testing of ground effect vehicles and wings in ground effect conducted by several researchers. This table provides useful information that will be used to compare the obtained results of the present study. The blockage area and the scaling factor have to be determined for the Air-Ray model and for the cases presented in Table 4 they are between 5\% and 7\%, which agrees with the recommendations discussed in Section 3.5. The scaling factor varies considerably depending of the model. Case 1 and 2 study two dimensional wings in ground effect, cases 3 to 5 study wing in ground effect (WIG) vehicles, which are vehicles that fly in ground effect (as the Air-Ray is designed, but usually they are powered). Case 6 studies a jet fighter when is flying in ground effect (usually at take off and landing). The smallest value of the reported scaling factors corresponds to case 1 which studies the front wing of a Formula 1 car, and the largest value corresponds to case 3 which studies a horizontal space launch vehicle, which is very large vehicle compared to the AirRay design. The vehicles that are closer in size to the Air-Ray are the ones reported in cases 4 and 5, which are WIG vehicles designed to transport passengers. Figure 23 [Seair Craft Inc., 2003] shows the test of the WIG vehicle described in case 6 of Table 4. 
Table 4. Test on ground effect used as a comparison cases.

\begin{tabular}{|c|c|c|c|c|c|}
\hline $\begin{array}{c}\text { Case } \\
\#\end{array}$ & Model Description & $\begin{array}{c}\text { Test Section } \\
\text { Characteristics } \\
\end{array}$ & $\begin{array}{l}\text { Blockage } \\
\text { area }\end{array}$ & $\begin{array}{l}\text { Scaling } \\
\text { factor }\end{array}$ & Author \\
\hline 1 & $\begin{array}{l}\text { Airfoil: NACA } 0015 . \\
\text { Chord length } 100 \mathrm{~mm} \text {. } \\
\text { Span } 300 \mathrm{~mm}\end{array}$ & $\begin{array}{l}\text { Square } 300 \times 300 \mathrm{~m} \\
\text { length }=1 \mathrm{~m} \\
\text { Free stream turbulence } \\
\text { intensity } 0.8 \% \text { at } 35 \mathrm{~m} / \mathrm{s} \\
\text { Fixed ground }\end{array}$ & $5 \%$ & - & $\begin{array}{l}\text { [Ahmed, } \\
\text { et al., } \\
\text { 2003] }\end{array}$ \\
\hline 2 & $\begin{array}{l}\text { Rectangular wing } \\
\text { Chord } 223.4 \mathrm{~mm} \\
\text { Span } 1100 \mathrm{~mm} \\
\text { Front wing of a Formula } \\
1 \text { car }\end{array}$ & $\begin{array}{l}2.1 \times 1.7 \mathrm{~m} \\
\text { Moving Belt } 3.5 \text { (long) } \times \\
1.5 \mathrm{~m} \text { (wide) }\end{array}$ & - & 1.25 & $\begin{array}{l}\text { [Zerihan, } \\
\text { et al., } \\
\text { 2000] }\end{array}$ \\
\hline 3 & $\begin{array}{l}\text { WIG horizontal space } \\
\text { launch vehicle } \\
\text { Airfoil: naca } 4415 \\
\text { Span } 533 \mathrm{~mm}\end{array}$ & $\begin{array}{l}\text { Round } 5 \mathrm{ft} \text { diameter, with } \\
\text { a flat plate to simulate the } \\
\text { ground. Fixed plate }\end{array}$ & $\begin{array}{l}\text { Less than } \\
7 \%\end{array}$ & 102 & $\begin{array}{l}\text { [Chawla, } \\
\text { et al., } \\
\text { 1990] }\end{array}$ \\
\hline 4 & WIG vehicle & Octagonal & - & 20 & $\begin{array}{l}\text { [Seair } \\
\text { Craft Inc., } \\
\text { 2003] }\end{array}$ \\
\hline 5 & $\begin{array}{l}\text { Reversed-delta wing } \\
\text { surface craft } \\
\text { Span } 400 \mathrm{~mm}\end{array}$ & $\begin{array}{l}1.15 \mathrm{~m} \times 0.89 \mathrm{~m} \times 1.5 \mathrm{~m} \\
\text { Octagonal. }\end{array}$ & - & $\begin{array}{l}7.7- \\
14.7\end{array}$ & $\begin{array}{l}\text { [Urquhart, } \\
\text { et al., } \\
\text { 2006] }\end{array}$ \\
\hline 6 & $\begin{array}{l}\text { Convair F-106B aircraft } \\
\text { Spam approx } 17.5 \mathrm{in}\end{array}$ & 36 in $x$ 51 in & - & 26 & $\begin{array}{l}\text { [Lee, et } \\
\text { al., 1989] }\end{array}$ \\
\hline
\end{tabular}

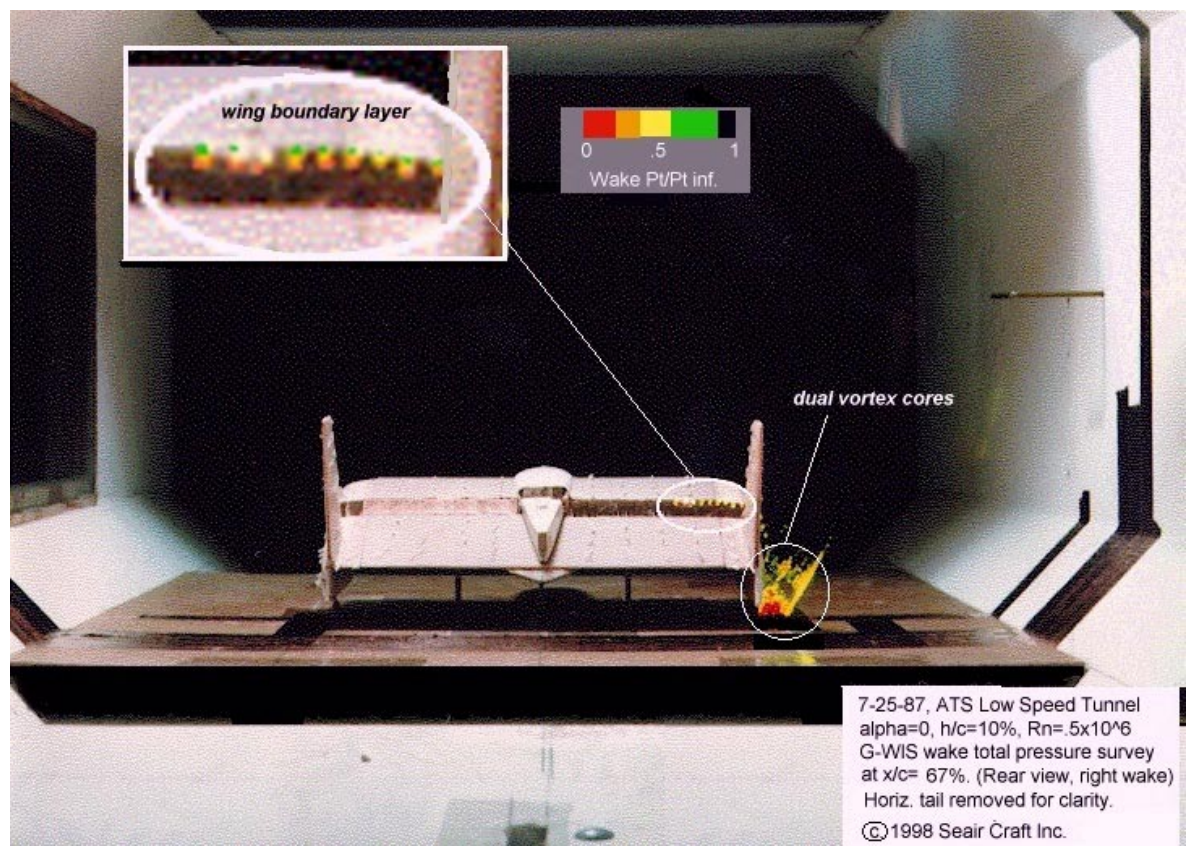

Figure 23. Testing of a wing in ground effect (WIG) vehicle [Seair Craft Inc., 2003] 


\section{TEST DESCRIPTION}

In this chapter the objectives of the Air-Ray wind tunnel tests are defined. A brief description of these tests is presented.

\subsection{TEST OBJECTIVES}

According to the classification of section 3.2 the initial tests that will be performed in the wind tunnel that is designed in this current work are dynamic and steady state tests, with a rigid free flying model. These tests are envisioned to provide:

- Qualitatively evaluate the dynamic stability

- Qualitatively evaluate the control characteristics

These envisioned tests to be performed with varying air velocity, slope angle, model trim, model weight, and control surface configuration. Also different models could be tested, with different aspect ratios and wing profiles.

With this evaluation it is expected to:

- Find out if the vehicle will work as expected

- Obtain the prototype optimum configuration (flap configuration, trim or angle of attack) that should be used for a specific operation (driver weight, slope).

- Obtain ways to improve the vehicle operation. 


\subsection{TEST PROCEDURE}

A typical test to evaluate the Air-Ray behavior will have the following primary steps:

- Set up the model for the test conditions (weight, flap configurations, trim)

- Mount the model in the wind tunnel

- Attach the cable to the model

- Initiate the airflow in the wind tunnel and bring it to the desired velocity.

- Initiate the ground simulator

- Check for correct position of the model and cable

- Initiate the video capture

- Tilt the wind tunnel to the desired angle

- Perform the test

- Return the wind tunnel to the horizontal position

- Turn the fan off

- Stop the video capture

- remove the model from the test section 


\section{REQUIREMENT LIST}

This chapter is a summary of the requirements for the new design, they are classified in requirements related to Geometry, Kinematics, Energy, Production and Operation. These are briefly explained below and summarized in Table 5.

The geometric requirements define the dimensions of the Air-Ray prototype and the geometric conditions that the new design has to met, which includes the geometry of the fan to be used. These conditions influence the size of the test section. The tilting angle and the available space to install the wind tunnel are also defined. As can be seen there is no limit with the height, since the wind tunnel can operate outside of the hangar. Although the use of a particular fan is imposed, it can be changed if it is impossible to correctly test the prototype with a wind tunnel constructed using that fan.

The kinematics requirements define the velocity of the prototype, which influences the required velocity (magnitude and quality) in the wind tunnel test section. Also the wind speed is defined. The energy requirements define the power required to operate the wind tunnel and the model. The current fan will generate air flow in the wind tunnel. Since the prototype is unpowered there is no need to power the model.

Finally the requirements related to production and operation define that the wind tunnel will be constructed and operated in the WVU hangar. This defines the available construction techniques which affects the design itself. At the place of operation dust may be present and precautions have to be taken in order to minimize its effect on the screens. When the tunnel is tilted, if it is too long, the roof can affect the flow at the test section, or in the worst case the tunnel can hit the roof. Fortunately, the tunnel anticipated location is close to one of the doors of the hangar, and so it can be operated partially outside (providing for example with wheels in order to facilitate its movement). 
Table 5. Requirement list for the wind tunnel design

\begin{tabular}{|c|c|}
\hline ID\# & Requirement \\
\hline \multicolumn{2}{|r|}{ GEOMETRY } \\
\hline 1 & $\begin{array}{l}\text { Fan outlet section dimensions: } \\
\text { • } \quad \text { Height H: } 667 \mathrm{~mm} \\
\text { - } \quad \text { Width W: } 597 \mathrm{~mm}\end{array}$ \\
\hline 2 & $\begin{array}{l}\text { Capable of holding a free flight model of a prototype with the specifications of Table } 1 . \\
\text { Maximize the size of the model. }\end{array}$ \\
\hline 3 & Tilting angle: at least $50^{\circ}$ \\
\hline 4 & Assigned Space length:10m, width: $3 \mathrm{~m}$ \\
\hline \multicolumn{2}{|r|}{ KINEMATICS } \\
\hline 5 & $\begin{array}{l}\text { Direction of the vehicle motion: Downwards (i.e. air is going upwards) } \\
\text { Prototype maximum velocity (relative to the ground): } 24.4 \mathrm{~m} / \mathrm{s} \\
\text { Wind Speed (updraft):0-4.2m/s }\end{array}$ \\
\hline 6 & $\begin{array}{l}\text { Non-uniformities of the airstream at the test section: } \pm 0.5 \% \text { velocity variation outside of } \\
\text { the boundary layers (as recommended [Mehta, et al., 1979]) }\end{array}$ \\
\hline \multicolumn{2}{|r|}{$\begin{array}{r}\text { ENERGY } \\
\end{array}$} \\
\hline 7 & Use the fan of the current wind tunnel. \\
\hline 8 & Prototype: unpowered \\
\hline \multicolumn{2}{|r|}{ PRODUCTION AND OPERATION } \\
\hline 9 & Possible to be constructed or modified at WVU hangar \\
\hline 10 & Use the parts of the current wind tunnel as possible \\
\hline 11 & Must operate at WVU hangar \\
\hline
\end{tabular}




\section{ANALYSIS OF ALTERNATIVES}

There are two basic configurations for the wind tunnel: open and closed return. Although a closed return wind tunnel requires less power to operate, and a better control over the flow can be obtained, they are more difficult to construct, and the tilting requirement increases even more the construction complexity and the costs. Also for small wind tunnels (as in the present case) the open configuration is recommended (see Section 3.1). Due to the above considerations an open return wind tunnel will be used.

There are two possible configurations for an open return wind tunnel: blowing mode and suction mode, these are shown in Figure 24 and Figure 25 and both of them use a centrifugal fan. The advantages and disadvantages of each mode or configuration are discussed below.

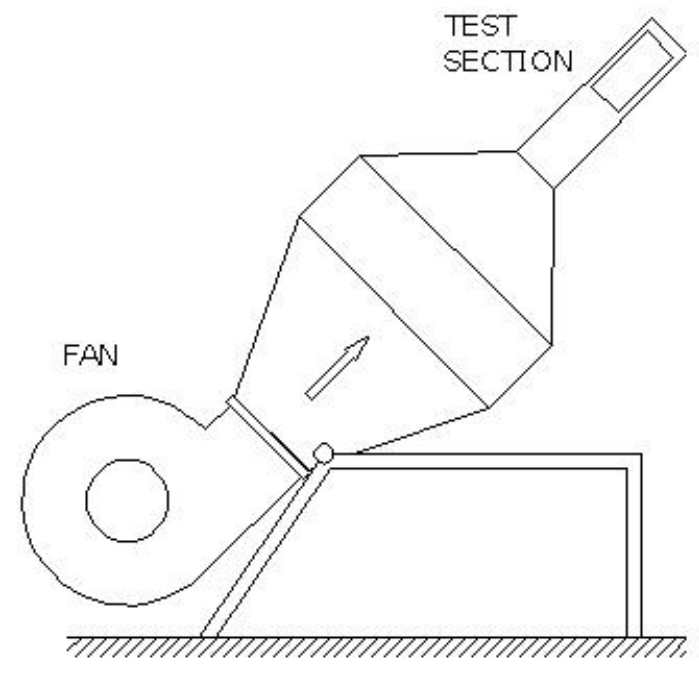

Figure 24. Blowing mode wind tunnel 


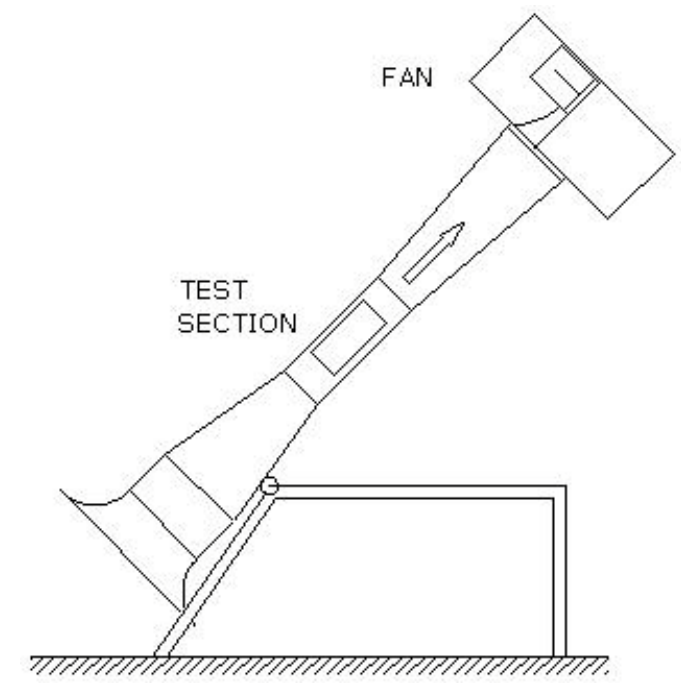

Figure 25. Suction mode wind tunnel

\subsection{BLOWING MODE}

\subsubsection{Advantages}

This configuration has been widely studied, recommended and used by Mehta (1977) and Mehta, et al. (1979) and thus there is available design information. The configuration is also favorable for the tilting operation, since the fan, which is the heaviest component, can be located close to the fulcrum and then not much power or torque for this operation will be required.

There are no problems associated with the suction, since the inlet is immediately prior to the impeller. Then it does not matter which conditions are at the inlet, since the impeller will change it, although a highly turbulent vortex flow will be the result. However it is recommended that the inlet of the fan will be located at distance greater than 1.5 times its diameter from a wall [Mehta, 1977].

The inlet conditions do not change with the tilting angle, since the inlet is perpendicular to the plane where the inclination occurs. All the sections of the wind tunnel can be rectangular, facilitating the construction process. 


\subsubsection{Disadvantages.}

Due to the non-uniformities of the flow at the exit of the fan, it is required to have more flow conditioners. The access to the test section is difficult for high tilt angles. A wide angle diffuser is required, and this can lead to flow separation, and non-uniformities at the test section.

\subsection{SUCTION MODE}

\subsubsection{Advantages}

Easier access to the test section, since when the tunnel tilts, the test section does not go too high as in the previous case. At the suction location the velocity profile has less turbulences and swirl, then less flow conditioners are required. With this configuration there is no need for a diffuser.

\subsubsection{Disadvantages}

If the fan is not close to the fulcrum the complexity of the construction is increased, since the fan has to be installed in a elevated position to allow the wind tunnel to tilt, i.e. the pivot axis has to be raised. If the fan is installed at the opposite end of the fulcrum as in Figure 25, the fan will go up and down when the tunnel is tilted and since there is a large distance the torque, or force, required to do this will be increased considerably, and also a stronger structure will be required.

A big bell-mouth inlet has to be constructed. Since the fan inlet is round, a component to change the shape from square to round has to be constructed. The condition at the inlet will severely affect the conditions at the test section, and even if uniform conditions are obtained at the inlet, when the tunnel is tilted due to the ground proximity, these conditions will change, and they will be difficult to control due to the wide range of the tilting angles. 


\subsection{SELECTION}

According to the previous discussion it is clear that the blowing configuration is the most favorable, and thus it will be used. The problem with the non-uniformities can be handled with flow conditioners treated in section 3.4.4. The problem with the access to the test section can be solved using video cameras, which capture, transmit and record the test in real time. 


\section{AERODYNAMIC DESIGN}

In this chapter the dimensions of the ducts and components related to the aerodynamic performance of the wind tunnel will be determined. At this point all that is known about the design is that it will be an open return wind tunnel with a centrifugal fan blowing air into the test section. To define the specific dimension of the components the methodology described in Chapter 2 will be used. Since the fan is already selected, only a brief discussion about the convenience of using this type of fan will be provided.

\subsection{TEST SECTION SHAPE}

As was described in Section 3.4.1.1 there are several options for the test section shape: circular, octagonal, square, rectangular, etc, and each one has its advantages. In this case it is desired to have a flat bottom, or floor in order to simulate the ground correctly. If for example a round section is used it will be necessary to install a flat board inside the tunnel and this will reduce the effective flow area, which will create problems related to the transition between the round section upstream of the test section and this new semi-round test section. A semi-round test section is not very common compared to, for example, a rectangular one. This has the advantage that there is available information related to their design, for example charts for data correction. This also allows the use of the wind tunnel for other tests (wings, fuselages, ground vehicles, etc), where a rectangular test section is more adequate and frequently used, so the data acquired is easier to correlate. An octagonal test section also has difficulties in the simulation of the flat ground.

Another important criterion for selecting the test section shape is related with the building effort. Clearly building a round test section requires a different 
manufacturing process than one with flat walls. If for example a circular test section will be used in order to maximize the flow uniformity, the contraction has to have this cross-sectional shape, a circular test section attached to a rectangular contraction with some adapter will not improve the flow quality since it is precisely at the contraction where a circular cross-section is required to improve flow quality. The construction of a contraction of this type will be a difficult task since it has curved walls (in longitudinal or axial section) and usually a mold will be required.

When constructing a tunnel with flat walls it is only necessary to cut and bond flat panels, usually of plywood, and for the contraction some bending is required, but the panels are still flat, i.e. they do not have curvature.

In this case the exit cross-section of the fan is rectangular, then it will be very convenient to conserve this shape along the wind tunnel (but it is not necessary to conserve the width to height ratio) to facilitate the construction process. Making the building process easier is emphasized due to the fact that this wind tunnel will be constructed by personal familiarized only with basic general construction techniques and using basic tools related to metalworking and woodworking. Then a rectangular cross-section along the tunnel will be used, in the next section the aspect ratio of the test section and the other components will be defined.

\subsection{TEST SECTION ASPECT RATIO (W/H)}

According to Barlow, et al. (1999) the aspect ratio of the test section can affect the magnitude of the downwash correction factor when testing wings, rotors and other lifting elements in the wind tunnel at the free air condition (i.e. out of ground effect). According to this reference the optimum value for the aspect ratio is 1.5 in order to minimize this correction factor. This value reduces the effects of the wind tunnel walls in the flow around the model (compared to the real flight conditions), especially the effect of the floor. When testing in ground effect the influence of the floor does not need to be corrected anymore since this 
is a real boundary that exists also in the real flight conditions of the prototype (i.e. the wind tunnel floor or bottom represents the ground surface), then the value of 1.5 does not have meaning for ground effect, however this value could be adopted for future uses of the wind tunnel as testing of wings and airplane models.

According to Section 3.4.1.2 test sections with aspect ratios of approximately 1.5 are very common and are often used in small wind tunnels. Then due to the above discussion, for the present case the value of the test section aspect ratio will be 1.5 .

\subsection{TEST SECTION BOUNDARIES}

In this case a closed test section will be used in order to avoid the construction of the sealed-off chamber around the open jet. To facilitate the access to the model a lateral hinged window will be used.

\subsection{FAN}

One of the most important questions that appeared when this work began was about the convenience of using a radial fan. This was due to the fact that axial fans are more often used in wind tunnels and in general centrifugal fans are recommended for operate at higher pressures and lower volumetric flow than axial fans. The conditions (low pressures and high volumetric rates) in a wind tunnel are favorable for axial fans.

The fan that will be used here is of the same type that is recommended by Mehta (1977): backward facing, aerofoil type with a single inlet. It is manufactured by Cincinnati fan, and the manufacturer's reference number is SQAF-270. It operates at a maximum speed of 1750rpm.

A bell-mouth at the fan inlet is recommended in order to have a uniform incoming flow, this bell-mouth is available from the manufacturer. 
As was discussed in section 3.4.3.4 the fan will be connected directly to a wide angle diffuser. Figure 26 [Cincinnati Fan, 2008] and Table 6 show the main dimension of the fan that will be used, including the dimension of the exit flange (this defines the inlet of the wide angle diffuser). The weight of the fan with the motor and driving system is $500 \mathrm{~kg}$. The detailed dimensions of this fan are in Appendix A.
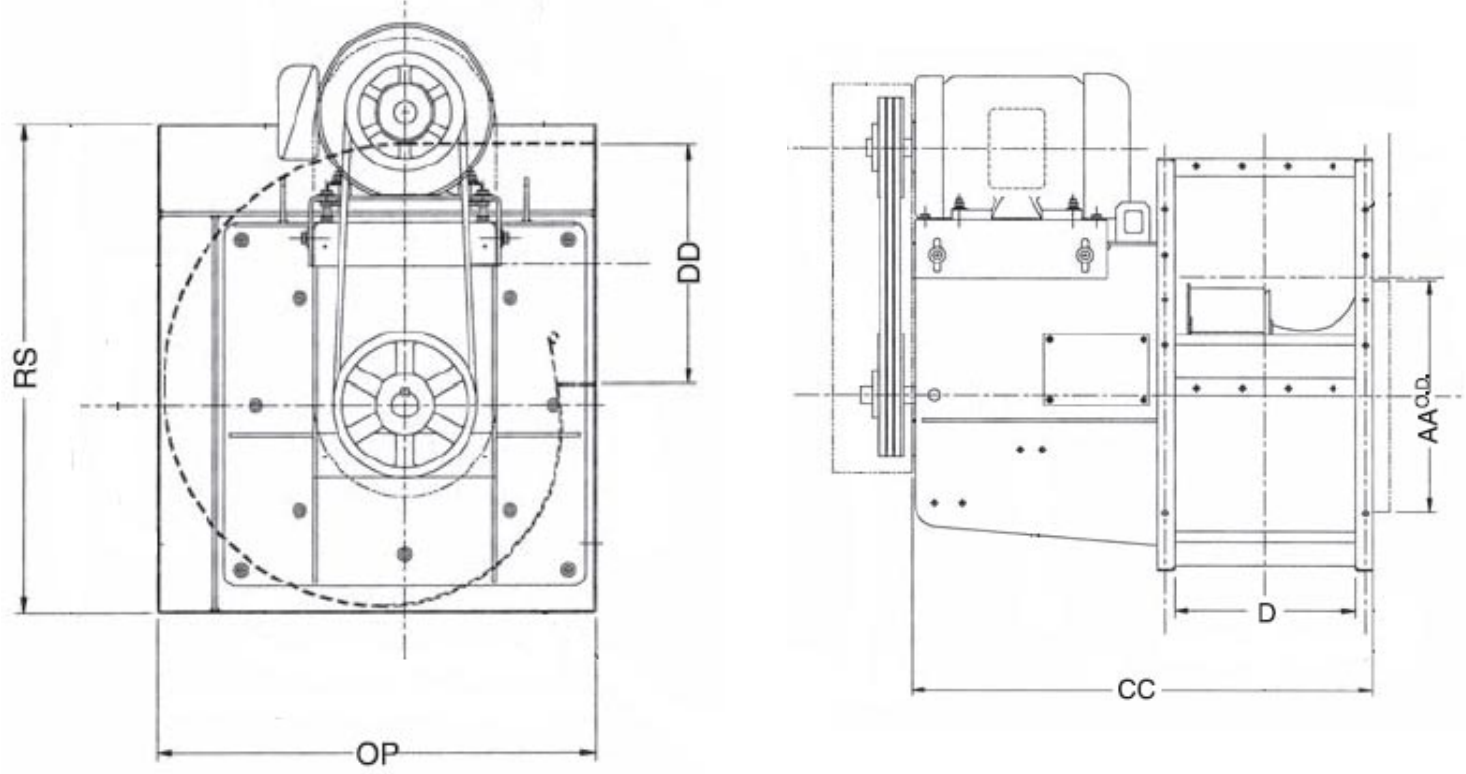

Figure 26. Fan dimensions, values on Table 6.

Table 6. Fan main dimension (in inches) according to Figure 26.

\begin{tabular}{|r|c|c|c|c|c|}
\hline RS & OP & DD & CC & D & AA \\
\hline $533 / 8$ & $471 / 2$ & $261 / 4$ & $5311 / 16$ & $231 / 2$ & $309 / 16$ \\
\hline
\end{tabular}

\subsection{WIDE ANGLE DIFFUSER}

For the present case a value of $A$ equal to five is chosen in order to maximize the test section size. This value has to be reduced if the fan can not maintain the required air velocity in the test section. With this value it is now possible to define the dimensions of the diffuser exit. 
In order to minimize the diffuser angle the exit of the diffuser has to be square, and the settling chamber has (or is convenient) to be square. The transition to the rectangular shape of the test section occurs at the contraction. According to this,

$$
H_{d, e}=W_{d, e},
$$

where, $W_{d, e}$ is defined as the width of the cross-section at the diffuser exit and $H_{d, e}$ is the height of the cross-section at the diffuser exit.

The dimensions of the diffuser inlet (same as the fan exit) are:

$$
H_{d, i}=667 \mathrm{~mm}
$$

and,

$$
W_{d, i}=597 \mathrm{~mm},
$$

where, $W_{d, i}$ is the width of the cross-section at the diffuser inlet and $H_{d, i}$ Height of the cross-section at the difusser inlet. Then the area $A_{d, i}$ of the diffuser inlet is:

$$
A_{d, i}=398199 \mathrm{~mm}^{2} .
$$

With the value of $A$, the area of the diffuser exit, $A_{d, e}$, can be obtained,

$$
A_{d, e}=5 A_{d, i}=1990995 \mathrm{~mm}^{2} .
$$

The height, $H_{d, e}$, and width $W_{d, e}$, of the exit section can be determined noting that,

$$
A_{d, e}=H_{d, e} \cdot W_{d, e},
$$

but since the diffuser exit is square,

$$
H_{d, e}=W_{d, e},
$$


then,

$$
A_{d, e}=H_{d, e}^{2}=1990995 \mathrm{~mm}^{2},
$$

finally,

$$
H_{d, e}=W_{d, e}=1411 \mathrm{~mm}
$$

these are also the dimensions of the settling chamber, and the contraction inlet. The next step is to define the diffuser expansion angle. Since this is a 3D diffuser $2 \theta$ will the larger between the angles, corresponding to the heights transitions (from 667 to 1411) and the angle corresponding to the width transition (from 597 to 1411). Clearly the larger will the one related to the width transitions, as is shown in Figure 27.

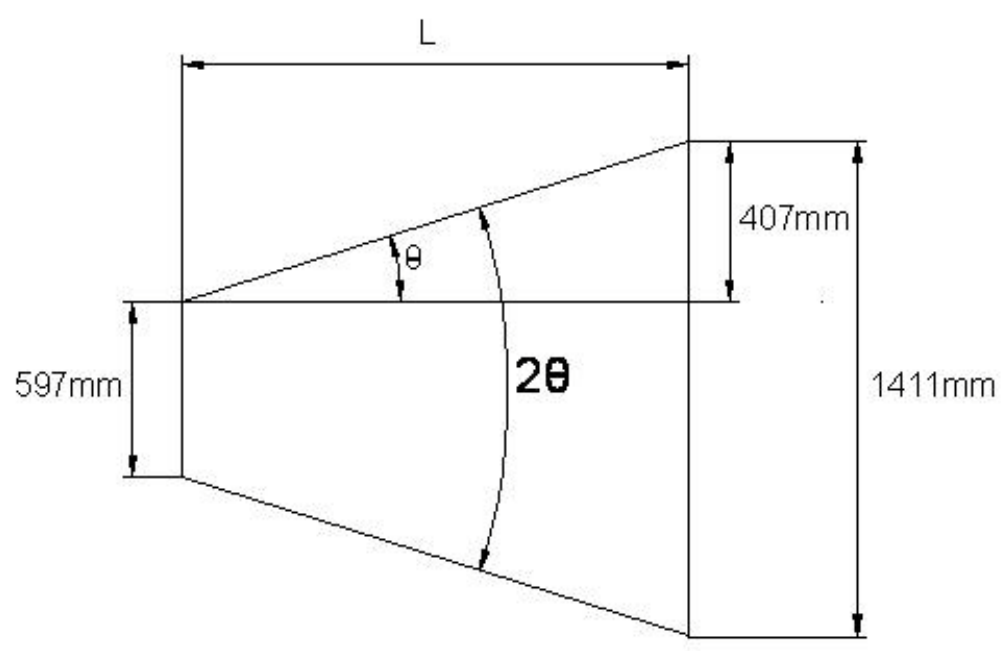

Figure 27. Top view of the wide angle diffuser

According to Figure 27, the expansion angle is,

$$
2 \theta=2 \cdot \arctan \left(\frac{407}{L_{d}}\right)
$$

Eq. 37

where $L_{d}$ is the diffuser length in millimeters. 
If a conventional diffuser $\left(2 \theta \approx 5^{\circ}\right)$ were used it would require a very long diffuser $\left(L_{d} \approx 9.3 \mathrm{~m}\right)$ resulting in a large wind tunnel that would not fit in the intended place of operation, particularly when tilted, thus a wide angle diffuser is needed.

As was discussed in 3.4.3.1 it is not recommended to have a large number of screens due to its tendency to trap dust. In this case two screens will be used. This selection and the value of five for $A$ will lead to an expansion angle between $25^{\circ}$ and $41^{\circ}$ (from the chart of Figure 10 [Mehta, 1977]). Table 7 shows the diffuser length according to these extreme values.

Table 7. Determination of the diffuser length

\begin{tabular}{|c|c|}
\hline $2 \theta$ & $L_{d}$ \\
\hline $25^{\circ}$ & $1836 \mathrm{~mm}$ \\
\hline $41^{\circ}$ & $1089 \mathrm{~mm}$ \\
\hline
\end{tabular}

There is no problem with either of these values for $L_{d}$ in relation to the space available, but it is preferable to have a lower expansion angle, since it means a less severe geometry in the diffuser which increases the probability of obtaining a successful operation with two screens. This component has a strong effect in the quality of the air at the test section so the value of $25^{\circ}$ is chosen.

\subsection{DIFUSSER SCREENS}

In this section the specifications of the two screens, that will be used to avoid separation, will be determined. In this case with $A$ equal to five, it is obtained from Eq. 4 that,

$$
K_{\text {sum }}>3.57 .
$$

Since two screens are used then,

$$
K_{s c r, d}>1.785 .
$$


Note that screens with equal pressure drop coefficient are used. With this value the parameters of the screens can be determined, and they will be completely defined. The values of these parameters are presented in Table 8, and were obtained from Table 3.

Table 8. Diffuser screen specifications

\begin{tabular}{|l|c|}
\hline Mesh Number & 22 \\
\hline Wire diameter & $0.345 \mathrm{~mm}$ \\
\hline$\beta$ open are ratio & 0.49 \\
\hline$K_{\text {scr, } d}$ & $1.788-1.912$ \\
\hline
\end{tabular}

As can be seen the value of the pressure drop coefficient does not have an exact value and does no match the one selected. This is because these values come from the commercially available screens, and also there are several definitions of $K_{s c r}$, but the values of this coefficient for the selected screens are larger than that determined by the calculations, and this is the condition that have to be met.

\subsubsection{Screen Position}

In this case only two screens are used, then from Eq. 10, Eq. 11, and Eq. 12:

$$
\begin{gathered}
M_{0}=2 K_{s c r, d}, \\
M_{1}=\frac{K_{s c r, d}}{L \cdot M_{0}}\left(x_{1}+x_{2}\right),
\end{gathered}
$$

and,

$$
M_{2}=\frac{K_{s c r, d}}{M_{0}}\left[\left(\frac{x_{1}}{L}-\frac{1}{2}\right)^{2}+\left(\frac{x_{2}}{L}-\frac{1}{2}\right)^{2}\right] .
$$


Since one of the screens is located at the inlet, or

$$
x_{1}=0 \text {, }
$$

then,

$$
M_{1}=\frac{K_{s c r, d}}{L \cdot M_{0}} x_{2},
$$

Eq. 43

and,

$$
M_{2}=\frac{K_{s c r, d}}{M_{0}}\left[\frac{1}{4}+\left(\frac{x_{2}}{L}-\frac{1}{2}\right)^{2}\right] .
$$

Since only the position of one screen is undetermined, only one equation (one parameter) is needed, in this case $M_{2}$ is used, with a value of 0.17 , then it is determined that,

$$
x_{2}=1469 \mathrm{~mm} \text {, }
$$

this is about $80 \%$ of the diffuser length.

\subsubsection{Screen Shape and Material}

In the diffuser curved screens will be used. These screens have to be curved in the two views (frontal and top, see Figure 28) then they will have curvature and since the commercially available screen are flat, they will have to be deformed to adapt to the required shape. For this reason is more convenient to use plastic screen (easier to deform). They are installed with the bending as indicated in Figure 28, which shows the frontal and top view of the diffuser. 


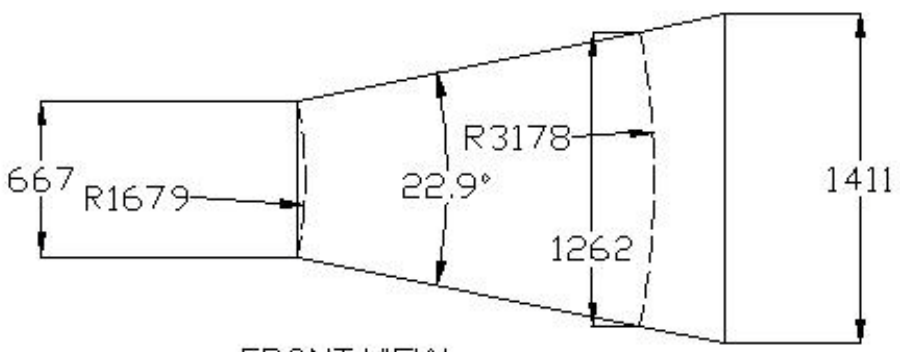

FRONT VIEW

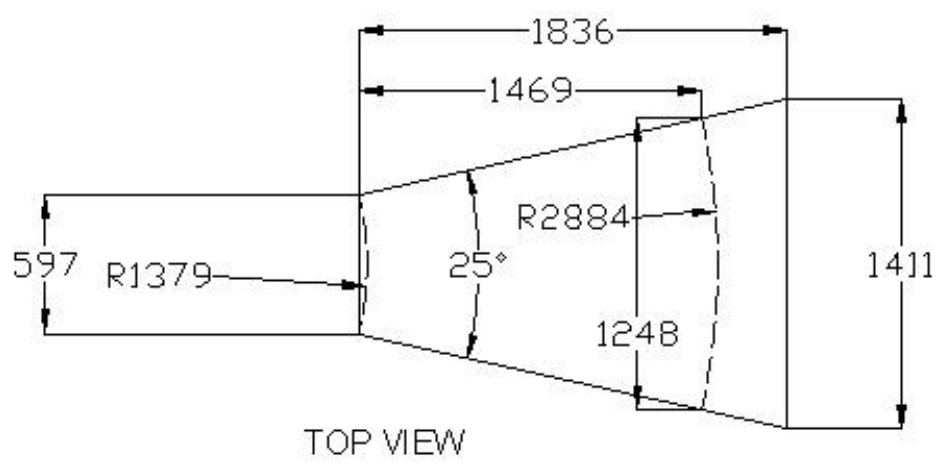

Figure 28. Location of the curved screens. Dimensions in $\mathbf{m m}$.

\subsection{SETTLING CHAMBER}

The settling chamber contains the flow conditioners: honeycomb and screens. In this case the main function of the screen is to create a uniform velocity profile and reduce turbulence. To completely specify the settling chamber it is necessary to define the number and parameters of the screens, the geometry of the honeycomb, and their position with respect to the settling chamber inlet. The cross-section of the settling chamber is the same as the diffuser exit i.e. a square section, thus,

$$
A_{S T C}=A_{d, e},
$$

where $A_{S T C}$ is the cross-sectional area of the settling chamber. 
For the Settling Chamber it is recommended to use two or three equal screens, with a $K_{s c r, s t c}$ equal to about 1.5 , in this case three screen will be used and with the recommended value of the pressure drop coefficient, then:

$$
K_{s c r, s t c} \approx 1.5 \text {. }
$$

With this value the specifications of these screen can be defined, they are shown in Table 9. The recommended distance between the screens is about 500 screen wire diameters. According to this recommendation and Table 9 the distance between the screens, $\Delta x_{s, s t c}$, is:

$$
\Delta x_{s, s t c}=209 \mathrm{~mm} \text {. }
$$

As was stated before the distance between the last screen and the exit of the settling chamber (contraction inlet) is recommended to be about 0.2 crosssection diameters. The cross-section diameter $D_{C S}$ is defined as the diameter of a circle with equal area to that of the rectangular cross-section:

$$
D_{C S}=2 \sqrt{\frac{A_{C S}}{\pi}},
$$

where $A_{C S}$ is the area of the cross-section in question and for this case is equal to $1990995 \mathrm{~mm}^{2}$ (same as the diffuser exit area), then,

$$
D_{C S}=1592 \mathrm{~mm} \text {, }
$$

and the distance between the last screen and the contraction inlet, $\Delta x_{i s, s t c}$ is,

$$
\Delta x_{i s, s t c}=318 \mathrm{~mm} .
$$


Table 9. Settling chamber screens specifications

\begin{tabular}{|l|c|}
\hline Mesh Number & 18 \\
\hline Wire diameter & $0.417 \mathrm{~mm}$ \\
\hline$\beta:$ open area ratio & 0.51 \\
\hline$K_{\text {scr, stc }}$ & $1.509-1.695$ \\
\hline Material & Steel \\
\hline
\end{tabular}

Metal screen are used for this case in order to reduce its deflection, since this type of screen tend to stay flatter than the plastics ones. It is necessary to determine the honeycomb specifications and its location. The recommended value for the honeycomb cell equivalent diameter is about $5 \mathrm{~mm}$, and the cell length to diameter ratio, $r_{H C}$, is defined as,

$$
r_{H C}=\frac{L_{H C}}{D_{H C}}
$$

where, $L_{H C}$ is defined as the length of the honeycomb cells and $D_{H C}$ is the diameter of a circle with equal area to that of a honeycomb cell.

The recommend value of $r_{H C}$ is between 7 and 10. The shape, and even the size, of the cells are not critical and can be chosen according to availability. This is all that is going to be specified about the honeycomb cells, its exact dimensions and shape will be determined when constructing the wind tunnel, according to availability and costs.

There are two recommended possibilities for the honeycomb position; it can be placed right at the settling chamber (just downstream of the diffuser exit), or it can be placed at a certain distance downstream of one screen, the other screens are placed downstream of the honeycomb. The second option is used when severe flow yaw angles are expected (greater that about $10^{\circ}$ ). In the present case, due to the flow that comes from the diffuser, this can be possible since the yaw angles are of the order of $12.5^{\circ}$ (half of the diffuser angle). Then a flat screen at the settling chamber inlet has to be installed. This screen is flat in order to deflect the flow toward the honeycomb cells i.e. create a flow parallel to 
the honeycomb cells. The distance between this screen and the honeycomb, $x_{H C}$, will be equal to 500 screen wire diameters from the diffuser exit, or

$$
x_{H C}=209 \mathrm{~mm} .
$$

This value is also used for the distance between the honeycomb and the next downstream screen. The total length of the settling chamber $L_{S T C}$ can now be determined, it is,

$$
L_{S T C}=x_{H C}+\Delta L_{H C}+2 \Delta x_{s, s t c}+\Delta x_{l s, s t c}
$$

and assuming a cell diameter of $5 \mathrm{~mm}$ and a $r_{H C}$ of 10 , the total length of the settling chamber would be,

$$
L_{S T C}=995 \mathrm{~mm}
$$

Figure 29 is a layout of the settling chamber and its components.

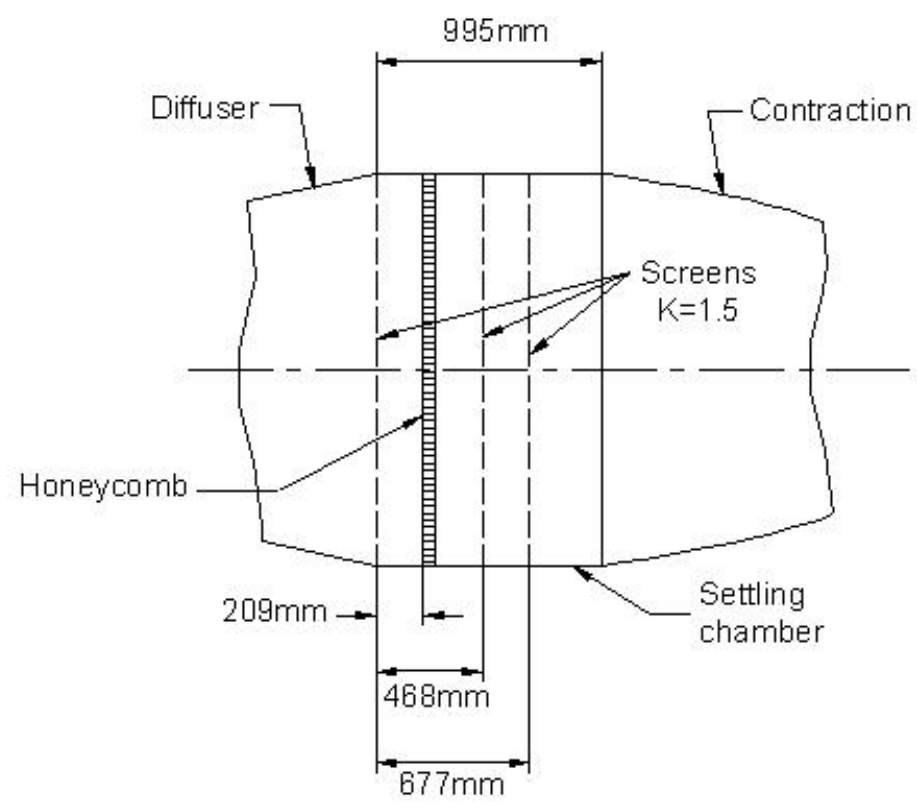

Figure 29. Screen and honeycomb position in the settling chamber. 
In order to the investigate the effect of the position of the honeycomb in the test section flow, it is recommended that the present design has the possibility to change the position of the screens and honeycomb, without any important modification, only assembly operations. It is also desirable that the design has the possibility to install more screens, or uninstall some of them, and for example investigate the operation with only two screens.

\subsection{CONTRACTION}

The main function of this component is to increase the uniformity of the flow and also to increase the magnitude of the velocity. As was discussed in section 3.4.5, the contraction ratio, $c$, is the principal parameter associated with this component and its value has to be chosen carefully. Furthermore since this component is the most difficult to construct, the importance of this selection is evident.

\subsection{CONTRACTION RATIO SELECTION}

The recommend values for, $c$, are between 6 and 9, and this is the range that will be considered in the selection. A low value of $c$ leads to a larger test section but low velocity, since the area increases. On the contrary a higher value of $c$ leads to a smaller test section, higher test section velocity and the most important a more uniform flow. The increase in the test section velocity as $c$ increases is not only due to the area reduction but also because the velocities upstream of the contraction are reduced which also reduces the pressure drop along the several components of the system (honeycomb, screens). This also reduces the fan pressure rise, and then more flow can be developed by the fan (see chapter 8).

Obviously a larger test section is an advantage, but so is a high velocity along with a more uniform flow, but since these advantages are for different values of $c$, more information is required in order to find an appropriate value. 
Figure 30 shows the fan and system pressure-flow plot (see chapter 8). Three different systems (wind tunnel with different contraction ratios) are shown with different values of $c$. The fan curve is at $1750 \mathrm{rpm}$, max speed. The point of operation of each system is where its curve intersects the one with the fan. From this figure the maximum test section velocity can be determined; these values are shown on Table 10.

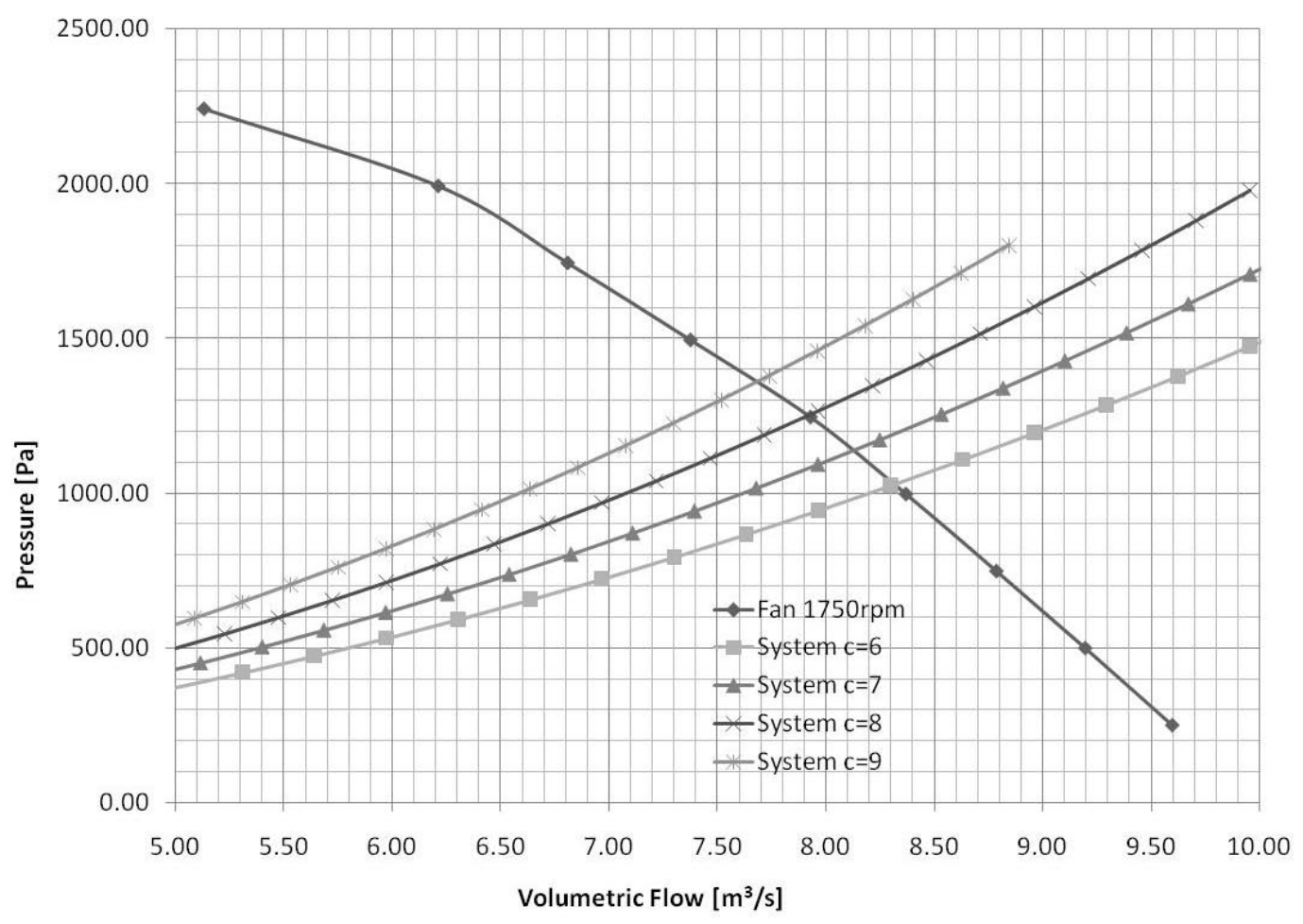

Figure 30. System curve for several contraction ratios

Table 10. Maximum velocity at the test section for several c

\begin{tabular}{|c|c|}
\hline$c$ & $U_{0}[\mathrm{~m} / \mathrm{s}]$ \\
\hline 6 & 25 \\
\hline 7 & 28.5 \\
\hline 8 & 31.7 \\
\hline 9 & 34.8 \\
\hline
\end{tabular}

As can be seen the minimum value of the velocity corresponding to a $c$ equal to six, is about $72 \%$ of the maximum value which correspond to $c$ equal to nine. Table 11 shows the values of several variables that depend on the 
contraction ratio which can be used to select its value. These variables are for the four different values of $c$.

Table 11 .Model parameter variation with c

\begin{tabular}{|r|r|r|r|r|}
\hline C & $\begin{array}{c}\text { Model } \\
\text { Scale } \\
\text { Lp/Lm }\end{array}$ & \multicolumn{1}{|c|}{$\begin{array}{c}\text { Model Spam } \\
{[\mathrm{m}]}\end{array}$} & \multicolumn{1}{c|}{$\begin{array}{c}\text { Model length } \\
\text { [m] }\end{array}$} & \multicolumn{1}{c|}{$\begin{array}{c}\text { V (required) } \\
\text { [m/s] }\end{array}$} \\
\hline 6 & 11.6 & 0.34 & 0.21 & 7.17 \\
\hline 7 & 12.5 & 0.32 & 0.20 & 6.90 \\
\hline 8 & 13.4 & 0.30 & 0.18 & 6.68 \\
\hline 9 & 14.2 & 0.28 & 0.17 & 6.48 \\
\hline
\end{tabular}

As can be seen from Table 11 the different values of the scale factors are adequate for testing these types of vehicles (see Table 4). With the data from Table 10 and Table 11 there is no a clear criteria for selecting $c$, i.e all of the values studied will work.

If the velocity profile at the contraction inlet were known, and its turbulence level, the variation in the inlet velocity, $\delta_{1}$, could be determined (see section 3.4.5). Using the recommended values for $\delta_{2}$ (about $\pm 0.5 \%$ outside the boundary layer [Mehta, 1979]) and the fact that the relation between $\delta_{1}$ and $\delta_{2}$ is proportional to the square of $c$ (Eq. 27), then, the value of $c$ would be selected as the minimum that meets this recommendation. Since the inlet velocity profile is unknown, it is not possible to follow this procedure, and since all the studied values work, a value of $c$ equal to nine will be used since it minimizes the velocity variation at the exit.

Although a study for finding the turbulence at the contraction inlet was not performed, Mehta (1977) suggests that by following its method the recommended flow quality can be obtained at the test section. A study like that will be difficult; although the fan exit velocity profile can be determined without major problems, the flow through the diffuser, screens and honeycomb will be too complex to simulate using CFD techniques. If experimental techniques were used they will have a cost on the same order as the wind tunnel, since all the components are required, and have to fit the fan. In order to avoid problems due 
to this unknown, ways to easily modify the wind tunnel components related to the flow quality will be provided in the design, for example adding more screens (see chapter 10).

With a contraction ratio of nine, the dimensions of the contraction exit can now be found. The dimensions (height $H_{c, i}$, width $W_{c, i}$ and area $A_{c, i}$ ) of the contraction inlet are equal to the dimension of settling chamber cross-section. The exit area, $A_{c, e}$, is equal to the area of the settling chamber divided by the contraction ratio:

$$
A_{c, i}=A_{S T C}
$$

and,

$$
A_{c, e}=\frac{A_{c, i}}{c},
$$

using the numerical values, the value obtained is,

$$
A_{c, e} \cong 221222 \mathrm{~mm}^{2} \text {, }
$$

this is also equal to,

$$
A_{c, e}=H_{c, e} \cdot W_{c, e},
$$

where $H_{c, e}$ and $W_{c, e}$ are the height and width of the contraction exit, respectively. But since the aspect ratio of the contraction exit is 1.5 then,

$$
W_{c, e}=1.5 H_{c, e},
$$

and,

$$
A_{c, e}=1.5 H_{c, e}^{2} \cong 221222 \mathrm{~mm}^{2} \text {, }
$$

then,

$$
H_{c, e}=384 \mathrm{~mm}
$$

and

$$
W_{c, e}=576 \mathrm{~mm} .
$$




\subsection{WALL SHAPE}

As was discussed in section 3.4.5.6 there are several methods to determine the wall shape. Mehta (1977) suggests a shape for a contraction with $c$ equal to nine, but with a constant aspect ratio of 1.33 , and in the present case there is a change in the aspect ratio (from 1 to 1.5) in the contraction. Nevertheless this contraction is reported as a successful configuration designed using the "eye-design" method.

In the present work, to determine the wall shape of the contraction, the method proposed by Su (1991) will be used. As was discussed in section 3.4.5.6 this method requires the definition of seven parameters $\left(L_{C} / D_{1 C}, X_{p}, c, A R_{1 C}\right.$, $\left.A R_{2 C}, n_{1}, n_{2}\right)$. Three of them are already defined (c,AR $\left.1 C, A R_{2 C}\right)$, and Su (1991) recommends the values of $n_{1}$ and $n_{2}$ as three and seven respectively. $D_{1 C}$ is already known and the value of $L_{C}$ is taken as the same that Mehta (1977) proposes, and for this case corresponds to $2026 \mathrm{~mm}$. Then the only parameter that has to be determined is $X_{p}$.

In order to find the value of $X_{p}$ a simulation in Fluent was conducted. Only one quarter of the model was used, as is shown in Figure 31. The inlet of the model was located at the position of the last screen in the settling chamber, and the exit is the contraction exit. Figure 31 also shows the boundary conditions, and at the inlet a constant velocity was assumed, at the exit an outflow condition was defined. The CFD had 73600 cell elements, $40 \times 40$ in a cross-section and 46 segments longitudinally (see Appendix B). The cell elements were smaller near of the walls, in order to have a better resolution in the boundary layers. Several cases were run in Fluent with different values of $X_{p}(0.3,0.4,0.46,0.5$, $0.6)$ and for three different inlet velocities $(0.3 \mathrm{~m} / \mathrm{s}, 0.7 \mathrm{~m} / \mathrm{s}$, and $3.9 \mathrm{~m} / \mathrm{s})$. The contraction proposed by Mehta was also run. Each case was run using a turbulent k-e RNG model. 


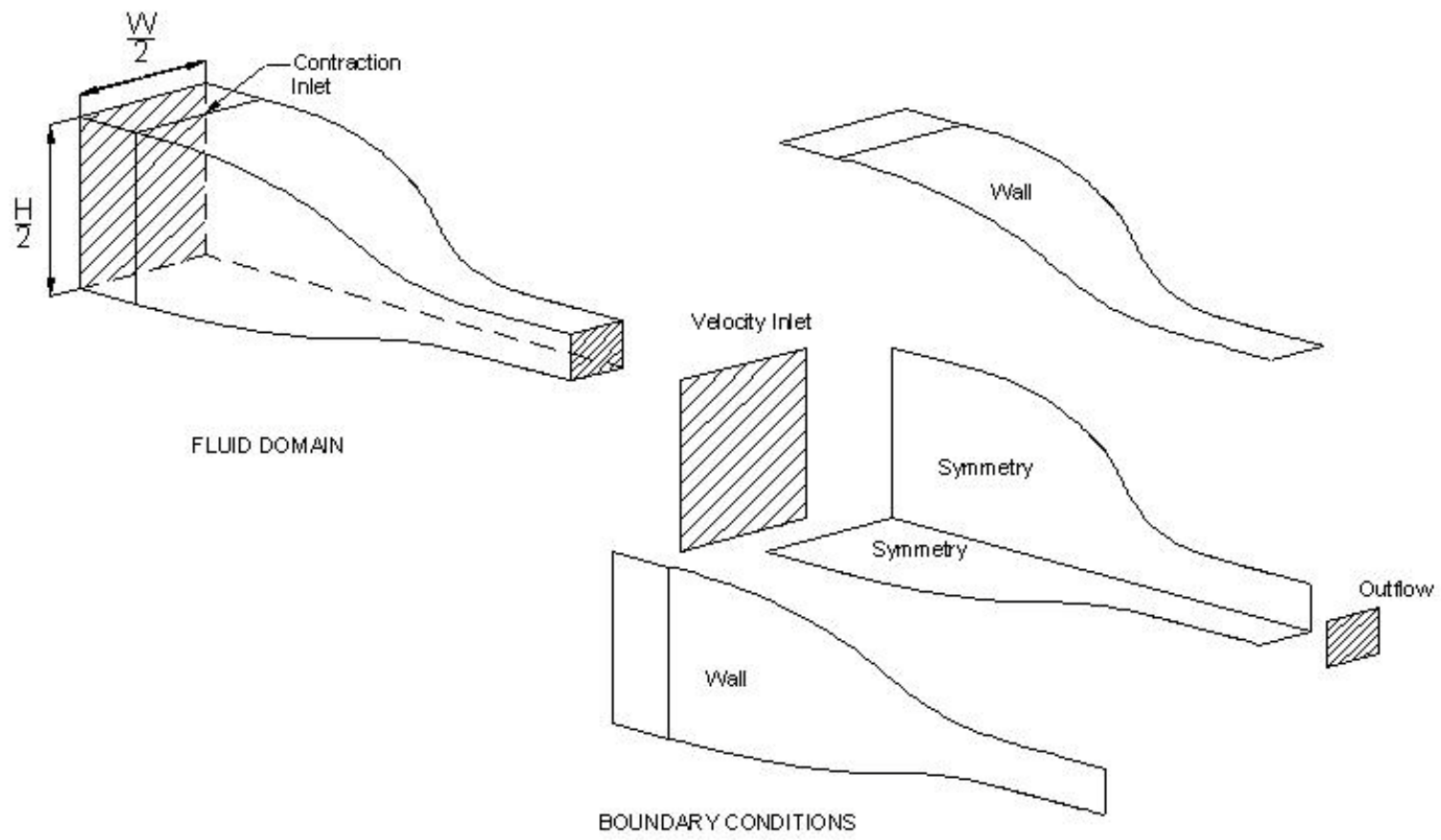

Figure 31. Fluid Domain and boundary conditions

Figure 32 shows the wall shapes of the contractions that were tested (due to the symmetry only a half of them are shown). It can be seen that there is a location from where the dimension of the contraction does change significantly (changes of less than $1 \mathrm{~mm}$ ) along the $x$-direction. The distance from the contraction inlet to this point is called the actual contraction length, and is reported in Table 12 for each case. 

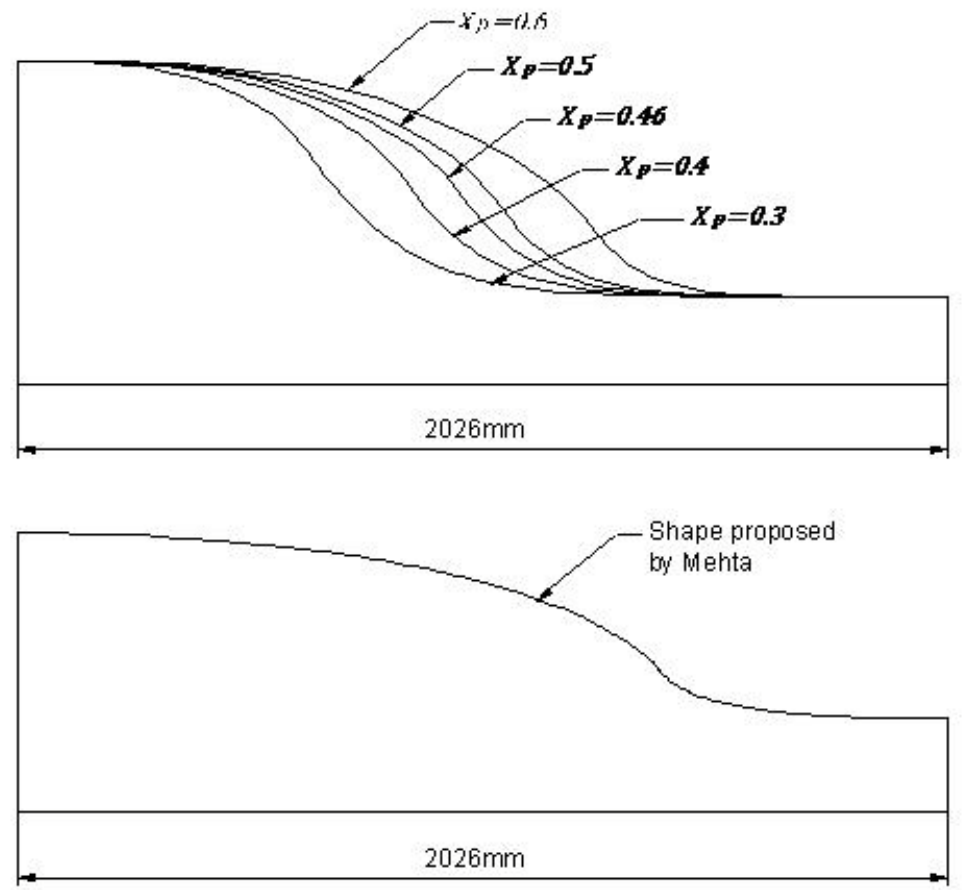

Figure 32. Studied Contraction wall shapes

Table 12. Contraction actual length

\begin{tabular}{|c|c|}
\hline$X_{p}$ & $L_{C}[\mathrm{~mm}]$ \\
\hline 0.3 & 1525 \\
\hline 0.4 & 1600 \\
\hline 0.46 & 1625 \\
\hline 0.5 & 1650 \\
\hline 0.6 & 1725 \\
\hline
\end{tabular}

In the Fluent simulations there was no separation predicted for each of the cases, including that proposed by Mehta (1977), plus the behavior of all of them was similar. The velocity profiles at the contraction exit (actual length) are presented on Appendix $\mathrm{C}$ and the pressure distribution in the contraction is presented in Appendix D these plots were obtained using Fluent.

The assumption of a constant velocity profile at the inlet of the fluid domain can be justified saying that this is a comparative study, which looks for contraction that reduces the non-uniformities at the exit. 
To determine the optimum value of $X_{p}$, the following variable, $\bar{u}_{c r}$, was defined as:

$$
\bar{u}_{c r} \stackrel{\text { def }}{=} \frac{u_{2 y, \text { max }}-u_{2 y, \text { min }}}{\langle u\rangle}+\frac{u_{2 z, \text { max }}-u_{2 z, \text { min }}}{1.5\langle u\rangle},
$$

where, according to the reference system of Figure 18,

$u_{2 y, \max }$ : is defined as the maximum velocity in the $\mathrm{YX}$ plane, at the actual contraction exit.

$u_{2 y, \text { min }}$ : is defined as the minimum velocity in the $\mathrm{YX}$ plane (outside of the boundary layer), at the actual contraction exit.

$u_{2 z, \max }$ : is defined as the maximum velocity in the ZX plane, at the actual contraction exit.

$u_{2 z, \min }$ : is defined as the minimum velocity in the $Z X$ plane (outside of the boundary layer), at the actual contraction exit

$\langle u\rangle$ : is defined as the mean velocity at the actual contraction exit

The first term of the right hand side of Eq. 53 corresponds to the maximum variation of the velocity profile relative to the average velocity at the $X Y$ plane (height) while the second is for the $X Z$ plane (width). The value of 1.5 that appears in the denominator of the second term of the right hand side of Eq. 53 correspond to the aspect ratio of the contraction exit, and it appears in this relationship to account for the fact that since the width of the exit section is greater than its height, so a variation of the same magnitude will be less critical for the width if this value does not appear. So, the variable $\bar{u}_{c r}$ accounts for the maximum variation of the velocity profile relative to the average velocity at two perpendicular planes.

The optimum value of $X_{p}$ was selected as the one that minimizes $\bar{u}_{c r}$, and in this case it was found to be 0.46 , as can be seen in Figure 33 , which shows the plot of $\bar{u}_{c r}$ against $X_{p}$ for several inlet velocities $(0.3 \mathrm{~m} / \mathrm{s}, 0.7 \mathrm{~m} / \mathrm{s}$, and $3.9 \mathrm{~m} / \mathrm{s})$. Figure 34 shows the velocity profiles for this case for an inlet velocity of $0.7 \mathrm{~m} / \mathrm{s}$. 


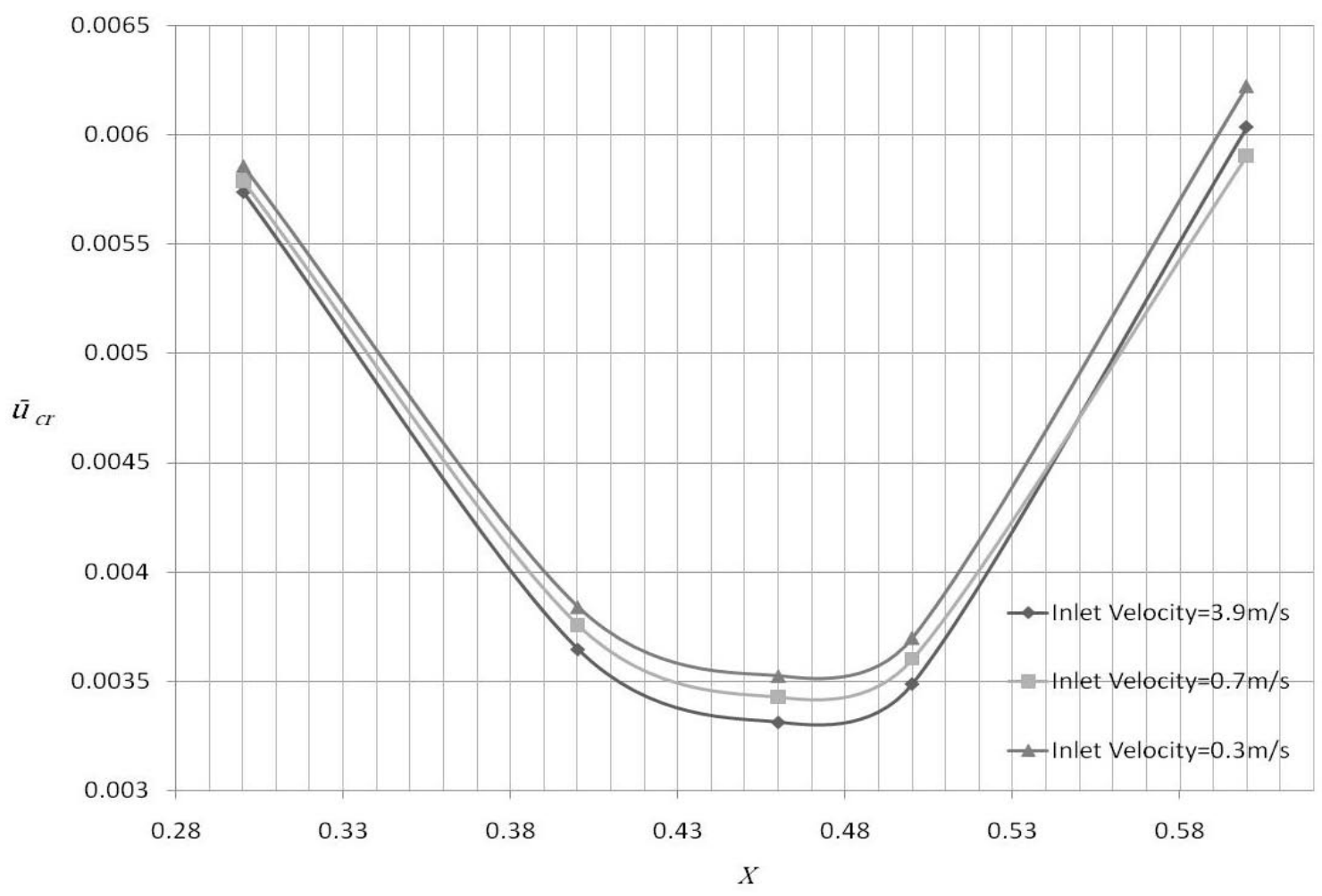

Figure 33. $\bar{u}_{c r}$ against Xp for the studied inlet velocities

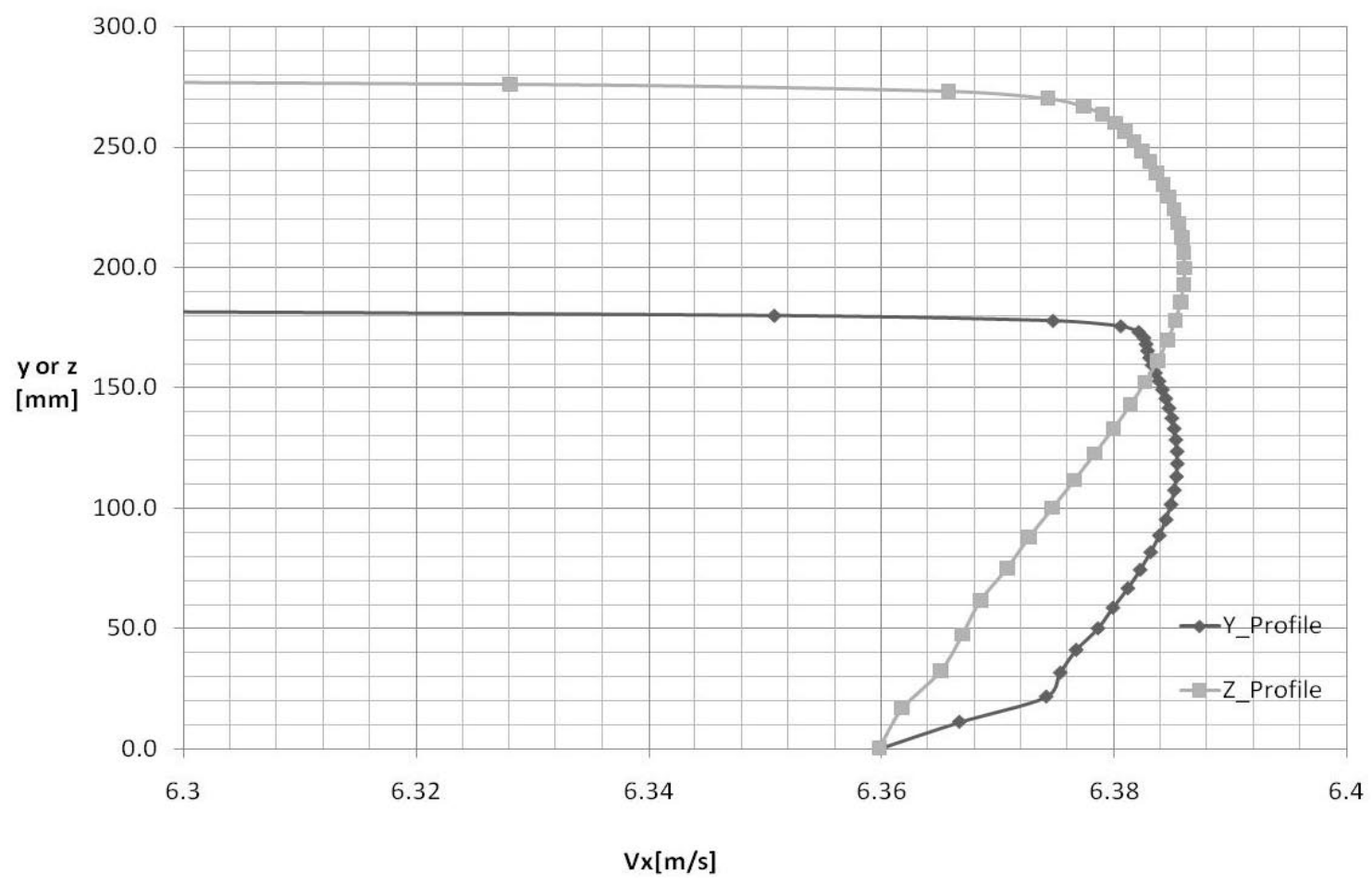

Figure 34. $X$-velocity at the contraction exit, at planes $X Y$ and $X Z$ for $X p=0.46$ and an inlet velocity of $0.7 \mathrm{~m} / \mathrm{s}$ 
Figure 35 shows a representation of the selected contraction. The coordinates of the selected wall shape are shown on Appendix E.

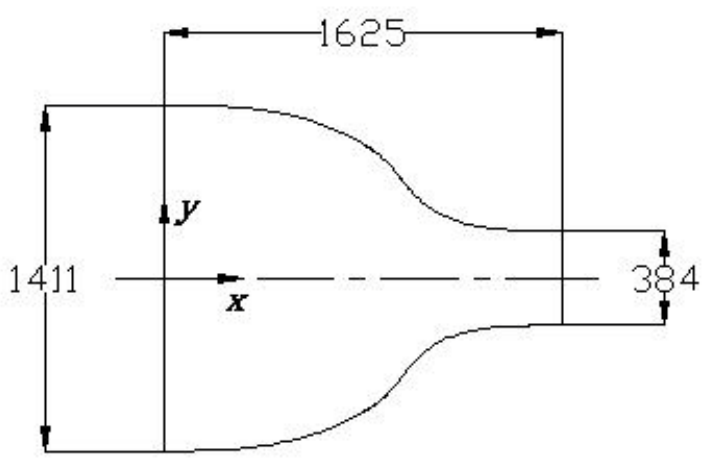

FRONT VIEW

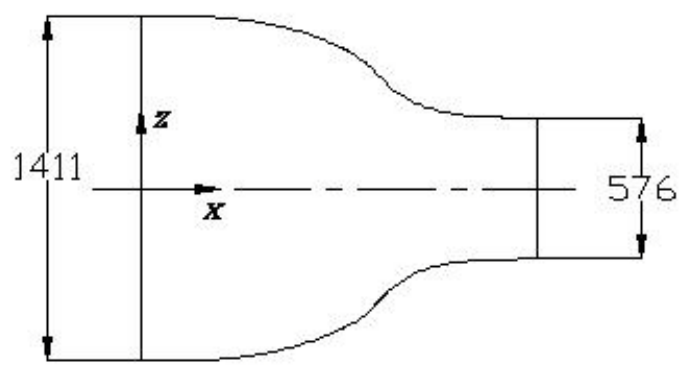

TOP VIEW

Figure 35. Wall shape of the selected contraction

\subsection{TEST SECTION}

The dimensions of the test section (height $H_{0}$, width $W_{0}$ and crosssectional area $A_{0}$ ) are equal to the dimensions of the contraction exit, or,

$$
\begin{aligned}
& H_{0}=384 \mathrm{~mm}, \\
& W_{0}=576 \mathrm{~mm},
\end{aligned}
$$

and,

$$
A_{0}=221222 \mathrm{~mm}^{2} \text {. }
$$


The length of the test section, $L_{0}$, is recommended to be between 1 and 1.5 times its width. In this case a value of 1.4 will be used, or,

$$
L_{0} \cong 800 \mathrm{~mm} \text {. }
$$

As was shown in section 3.4.1.4 it is recommended that the distance between the contraction outlet and the test section inlet be about 0.5 diameters after the contraction. According to this the test section starts at $266 \mathrm{~mm}$ downstream of the contraction. Then the total length of the exit duct after the contraction, $L_{E D}$, is,

$$
L_{E D}=1066 \mathrm{~mm} \text {. }
$$

\subsection{SUMMARY}

Table 13 and Figure 36 show a summary of the dimensions of the wind tunnel components that were derived in the previous sections. As can be seen the total length of the ducts is $5522 \mathrm{~mm}$.

\begin{tabular}{|c|c|c|}
\hline \multicolumn{3}{|c|}{ DIFFUSER } \\
\hline \multirow[t]{3}{*}{ Inlet } & $H_{d, i}:$ Height & $667 \mathrm{~mm}$ \\
\hline & $W_{d, i}:$ Width & $597 \mathrm{~mm}$ \\
\hline & $A_{d, i}:$ Area & $398199 \mathrm{~mm}^{2}$ \\
\hline \multirow[t]{3}{*}{ Exit } & $H_{d, e}:$ Height & $1411 \mathrm{~mm}$ \\
\hline & $W_{d, e}:$ Width & $1411 \mathrm{~mm}$ \\
\hline & $A_{d, e}:$ Area & $1990995 \mathrm{~mm}^{2}$ \\
\hline \multicolumn{2}{|c|}{$A$ : Area Ratio } & 5 \\
\hline \multicolumn{2}{|c|}{ 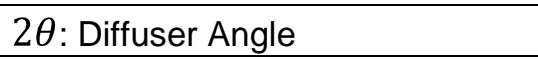 } & $25^{\circ}$ \\
\hline \multicolumn{2}{|c|}{ Diffuser Length } & $1836 \mathrm{~mm}$ \\
\hline \multicolumn{2}{|c|}{ Wall shape } & Straight walls \\
\hline \multirow[t]{8}{*}{ Screens } & Number of screens & 2 Screens \\
\hline & Mesh number & \#22 \\
\hline & Wire diameter & $0.345 \mathrm{~mm}$ \\
\hline & $\beta$ :open area ratio & 0.49 \\
\hline & $K_{s c r, d}$ & $1.788-1.912$ \\
\hline & Positioning & $x_{1}=0, x_{2}=1469 \mathrm{~mm}$ \\
\hline & Screen Shape & Curved screens \\
\hline & Material & Plastic (nylon or polyester) \\
\hline
\end{tabular}

Table 13. Dimensions of the Wind Tunnel components 
Table 13. Dimensions of the Wind Tunnel components (cont)

\begin{tabular}{|c|c|c|}
\hline \multicolumn{3}{|c|}{ SETTLING CHAMBER } \\
\hline \multirow[t]{3}{*}{ Cross-Section } & $H_{s t c}:$ Height & $1411 \mathrm{~mm}$ \\
\hline & $W_{\text {stc }}:$ Width & $1411 \mathrm{~mm}$ \\
\hline & $A_{s t c}:$ Area & $1990995 \mathrm{~mm}^{2}$ \\
\hline \multicolumn{2}{|l|}{$L_{s t c}$, Length } & $995 \mathrm{~mm}$ \\
\hline \multirow[t]{8}{*}{ Screens } & Number of screens & 3 Screens \\
\hline & Mesh number & $\# 18$ \\
\hline & Wire diameter & $0.417 \mathrm{~mm}$ \\
\hline & $\beta$ :open area ratio & 0.51 \\
\hline & $K_{s c r, s t c}$ & 1.509-1.695 \\
\hline & Positioning & $x_{1}=0, x_{2}=468 \mathrm{~mm}, x_{3}=677 \mathrm{~mm}$ \\
\hline & Screen Shape & Flat screens \\
\hline & Material & Steel \\
\hline \multirow[t]{4}{*}{ Honeycomb } & $D_{H C}$ & $5 \mathrm{~mm}$ \\
\hline & $L_{H C}$ & $50 \mathrm{~mm}$ \\
\hline & $K_{H C}$ & 0.5 \\
\hline & Position & $x_{H C}=209 \mathrm{~mm}$ \\
\hline \multicolumn{3}{|c|}{ CONTRACTION } \\
\hline \multicolumn{2}{|l|}{ Contraction ratio } & 9 \\
\hline \multirow[t]{3}{*}{ Inlet } & $H_{C, i}:$ Height & $1411 \mathrm{~mm}$ \\
\hline & $W_{C, i}:$ Width & $1411 \mathrm{~mm}$ \\
\hline & $A_{C, i}:$ Area & $1990995 \mathrm{~mm}^{2}$ \\
\hline \multirow[t]{3}{*}{ Exit } & $H_{C, e}:$ Height & $384 \mathrm{~mm}$ \\
\hline & $W_{C, e}:$ Width & $576 \mathrm{~mm}$ \\
\hline & $A_{C, e}:$ Area & $221222 \mathrm{~mm}^{2}$ \\
\hline \multicolumn{2}{|c|}{$L_{C}$, Contraction Length } & $1625 \mathrm{~mm}$ \\
\hline \multicolumn{2}{|c|}{ Wall shape } & $\begin{array}{c}\text { Curved (inlet } 3^{\text {rd }} \text { order polynomial, outlet } 7^{\text {th }} \text { order } \\
\text { polynomial, transition at } X=0.46 \text { ) }\end{array}$ \\
\hline \multicolumn{3}{|r|}{ TEST SECTION } \\
\hline \multicolumn{2}{|l|}{$H_{0}:$ Height } & $384 \mathrm{~mm}$ \\
\hline \multicolumn{2}{|l|}{$W_{0}:$ Width } & $576 \mathrm{~mm}$ \\
\hline \multicolumn{2}{|l|}{$A_{0}:$ Area } & $221222 \mathrm{~mm}^{2}$ \\
\hline \multicolumn{2}{|c|}{$L_{0}:$ Length } & $800 \mathrm{~mm}$ \\
\hline \multicolumn{2}{|c|}{$L_{E D}:$ Total duct length after contraction } & $1066 \mathrm{~mm}$ \\
\hline \multicolumn{2}{|c|}{ Aspect ratio } & 1.5 \\
\hline
\end{tabular}




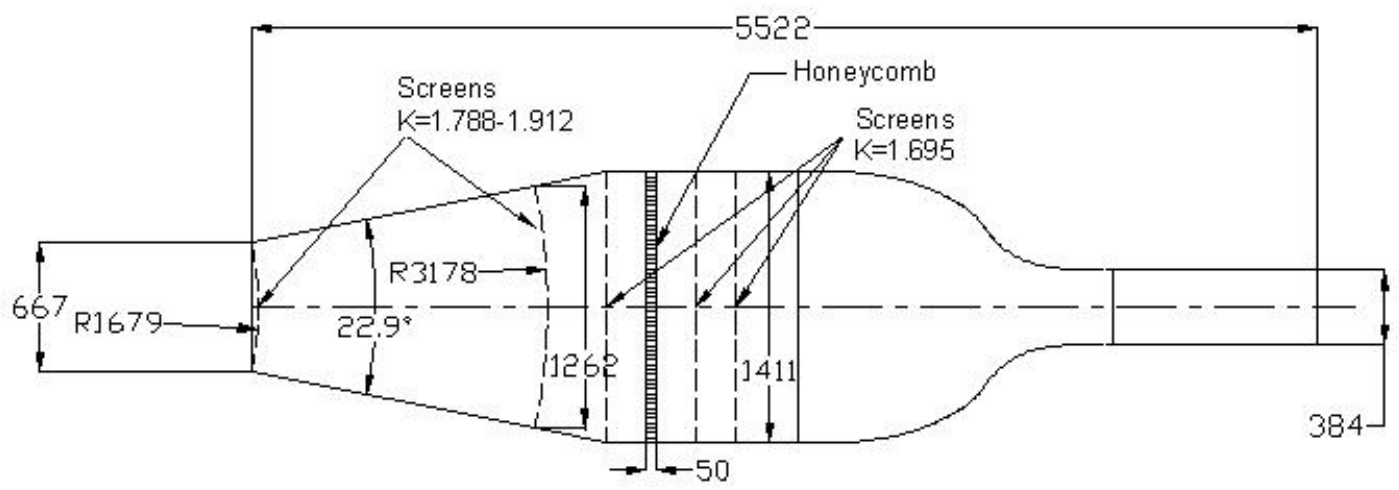

FRONT VIEW

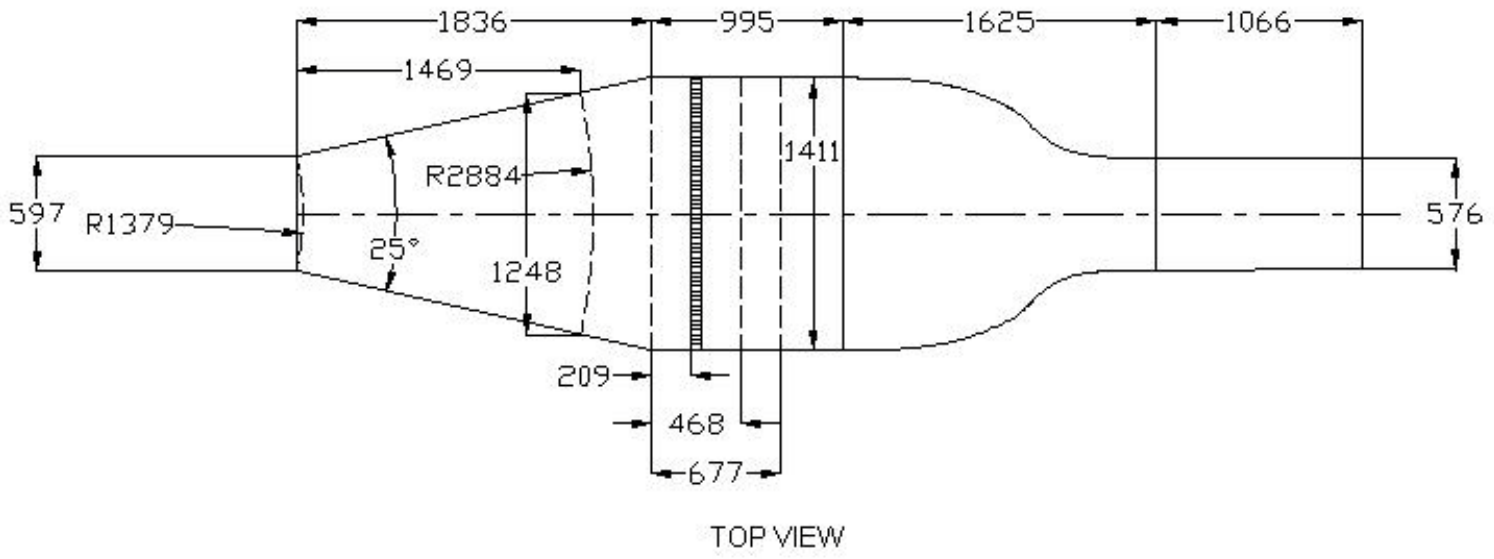

Figure 36. Dimensions of the ducts (in $\mathrm{mm}$ ) 


\section{MAXIMUM TEST SECTION VELOCITY}

In this chapter the maximum velocity that can be obtained with the fan and the designed wind tunnel will be obtained. Figure 37 shows a schematic representation of the static and total pressure distribution along the different components of the wind tunnel (Figure 39 shows the pressure distribution for the maximum obtainable velocity). They are different for each component due to the fan effect, the changing duct cross-sectional area, and the pressure losses due to the viscous effects. As can be seen the inlet is at atmospheric pressure, which is taken as a reference pressure (so the pressure shown in the diagram will be the gage pressure). There is a pressure rise due to the fan affect. The crosssectional area increases along the diffuser, this increases the static pressure (pressure regain) and decreases the velocity along the diffuser reducing the dynamic pressure, but due to the pressure losses (viscous effects) the total pressure decreases. There are local pressure losses across the honeycomb and the screens, as can be seen the larger localized loss of pressure occurs at the first screen, which is located at the fan exit which has a higher velocity than the settling chamber velocity. In the settling chamber the main pressure losses are due to the three screens and the honeycomb, and there are minor losses due to the wall effect (skin friction). The contraction reduces the static pressure increasing the dynamic pressure, but the total pressure is reduced due to the viscous effects. Finally at the test section the pressure is reduced mainly due to the skin friction. 


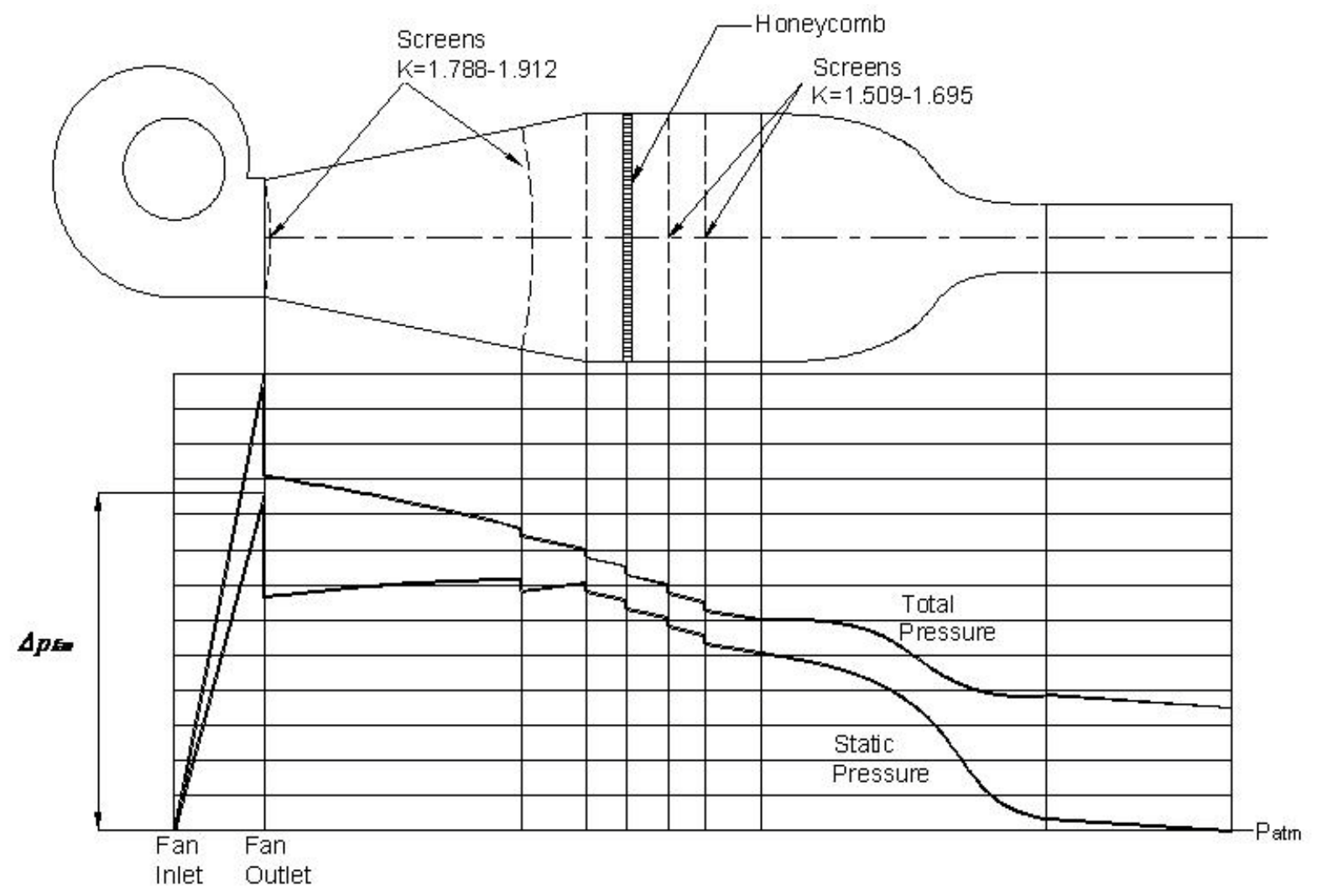

Figure 37. Schematic static and total pressure distribution along the wind tunnel.

\subsection{SYSTEM PRESSURE DISTRIBUTION}

As can be seen from Figure 37 the total change in pressure along the wind tunnel is equal to the summation of the pressure changes across each component. The pressure change in each component is due to pressures losses, for the diffuser and the contraction there is an additional change in pressure due to the variation of their cross-sectional areas. For the screens and the honeycomb the pressures losses can be considered localized (since the length of this components are small compared to the wind tunnel length), in the other components these pressure losses are distributed (along the component). The pressure losses for each component can be related to the velocity (dynamic pressure) at its inlet with the following equation, 


$$
\Delta p_{\text {loss }, i}=\frac{1}{2} \rho K_{i} V_{i}^{2},
$$

where $K_{i}$ is the pressure loss constant of each component. Due to the different sizes of the cross-section along the tunnel (which leads to different velocities in the component), it is convenient to relate all of these pressure losses to the test section velocity, and then a new constant is defined as:

then,

$$
K_{0, i}=\frac{\Delta p_{i}}{\frac{1}{2} \rho U_{o}^{2}}=K_{i}\left(\frac{A_{0}}{A_{i}}\right)^{2},
$$

Eq. 55

$$
\Delta p_{\text {loss }, i}=\frac{1}{2} \rho K_{0, i} U_{0}^{2} .
$$

Eq. 56

In the following sections each $K_{0, i}$ and the pressure changes in each component are determined.

\subsubsection{Test Section Pressure Change}

At this component the pressure losses are basically due to the skin friction, according to Mehta, et al. (1979) they can be estimated using the following expression:

$$
\Delta p_{0} \cong-0.07 \cdot \frac{1}{2} \rho U_{0}^{2} .
$$

Eq. 57

\subsubsection{Contraction Pressure Change}

The pressure change in the contraction is due to the area reduction and pressure losses, or,

$$
\Delta p_{C}=\frac{1}{2} \rho \frac{U_{o}^{2}}{c^{2}}\left[1-c^{2}-K_{0, c}\right],
$$


rearranging,

$$
\frac{\Delta p_{C}}{\frac{1}{2} \rho U_{o}^{2}}=\left[\frac{1}{c^{2}}-1-K_{0, c}\right]
$$

from the CFD simulation it was obtained that,

$$
K_{0, c}=0.123,
$$

then from Eq. 59,

$$
\Delta p_{C} \cong-1.111 \cdot \frac{1}{2} \rho U_{0}^{2} .
$$

Eq. 60

The pressure distribution in the contraction is shown in Appendix E.

\subsubsection{Settling Chamber Pressure Change}

For this component $K_{0}$ is determined considering the pressure losses due to the screens and honeycomb, or

$$
K_{0, s t c}=K_{H C}\left(\frac{A_{0}}{A_{s t c}}\right)^{2}+3 K_{s c r, s t c}\left(\frac{A_{0}}{A_{s t c}}\right)^{2}
$$

using the values of Table 13 it is obtained that,

$$
K_{0, s t c}=0.069 \text {, }
$$

then,

$$
\Delta p_{s t c} \cong-0.069 \cdot \frac{1}{2} \rho U_{0}^{2} .
$$

Note that the pressure losses due to the skin friction (with the settling chamber walls) are not considered, since they are small compared to the pressure losses due to the screens. 


\subsubsection{Diffuser Pressure Change}

In this case $K_{0}$ is giving by the pressure losses due to the diffuser screens,

$$
K_{0, d}=K_{S 1}\left(\frac{A_{0}}{A_{S 1}}\right)^{2}+K_{S 2}\left(\frac{A_{0}}{A_{S 2}}\right)^{2},
$$

Eq. 63

using the values of Table 13 and Figure 36, it is obtained that,

$$
K_{0, d}=0.63,
$$

then,

$$
\Delta p_{d} \cong-0.63 \cdot \frac{1}{2} \rho U_{0}^{2}+\Delta p_{\text {Regain }}
$$

Eq. 64

where $\Delta p_{\text {Regain }}$ is the pressure rise in the diffuser due to the increment in the cross-sectional area. Usually $\Delta p_{\text {Regain }}$ is neglected (this makes the calculated pressure slightly larger than the actual required pressure), then,

$$
\Delta p_{d} \cong-0.63 \cdot \frac{1}{2} \rho U_{0}^{2} .
$$

Eq. 65

The system pressure can now be determined. From Figure 37 , it can be seen that the total change in pressure along the wind tunnel has to be equal to the pressure change across the fan, $\Delta p_{f a n}$, thus,

$$
\Delta p_{\text {fan }}=\Delta p_{d}+\Delta p_{s t c}+\Delta p_{C}+\Delta p_{0}
$$

Eq. 66

inserting Eq. 57, Eq. 60, Eq. 62, Eq. 65 in this expression, gives,

$$
\Delta p_{\text {fan }}=1.88 \frac{1}{2} \rho U_{0}^{2} .
$$

Eq. 67

This is the required change in static pressure through the fan. The volumetric flow, $\dot{\forall}$, is given by 


$$
\dot{\forall}=A_{0} U_{0} \text {, }
$$

Eq. 68

inserting this and the value of $A_{0}$ into Eq. 68, gives,

$$
\Delta p_{\text {fan }}=23.05 \dot{\forall}^{2}
$$

Eq. 69

with this equation the operational curve of the system (ducts, screens, etc) can be plotted, it can be seen in Figure 38 .

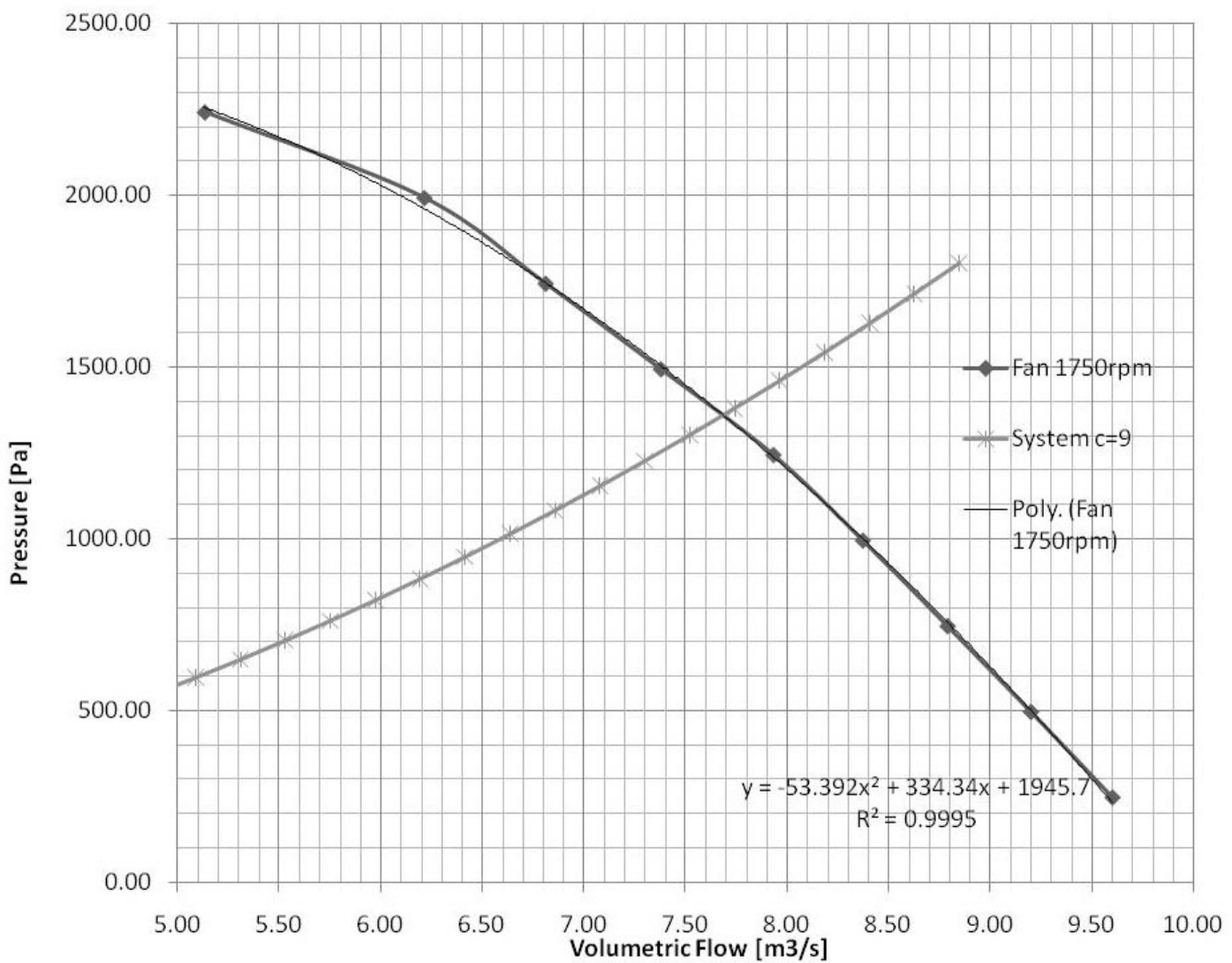

Figure 38. Fan and system pressure vs. volumetric flow curve (fan at max speed: 1750rpm) 


\subsection{FAN WORKING POINT AT MAXIMUM POWER}

In this section the working point of the fan will be determined, i.e., the maximum volumetric flow (and its associated fan static pressure) that can be generated by the fan when installed in the wind tunnel. The American Conference of Governmental Industrial Hygienists (1976) defines Fan total pressure, FanTP, as "the increase in total pressure through or across the fan", which is expressed by,

$$
\text { FanTP }=p_{t, f, e}-p_{t, f, i},
$$

Eq. 70

where, $p_{t, f, i}$ is the total pressure at the fan inlet and $p_{t, f, e}$ is the total pressure at the fan exit. The Fan Static pressure, FanSP, is defined by,

$$
F a n S P=F a n T P-D P_{f, e},
$$

where $D P_{f, e}$ is the dynamic pressure at the fan exit. The Fan Static Pressure is the pressure that is usually specified by the fan manufacturer, and is specified in charts or tables as a function of the air flow, and fan RPM. Figure 38 shows this curve for the fan that is used in this case, which was plotted using the manufacturer data [Cincinnati Fan, 2008]. This curve is for the fan rotating at the maximum speed (1750rpm), and can be approximated by the expression given in Eq. 72 (as can be seen in Figure 38),

$$
\text { FanSP }=-53.392 \dot{\forall}^{2}+334.34 \dot{\forall}+1945.7 .
$$

Inserting Eq. 70 into Eq. 71 yields,

$$
\operatorname{FanSP}=p_{t, f, e}-p_{t, f, i}-D P_{f, e}
$$

and since, in general,

$$
p_{t}=p+D P,
$$


then,

$$
F a n S P=p_{f, e}-p_{f, i}-D P_{f, i}
$$

Eq. 75

where $p_{f, e}$ is the static pressure at the fan exit and, in this case, $p_{f, i}$ (static pressure at the fan inlet) and $D P_{f, i}$ (dynamic pressure at the fan inlet) are both equal to zero since the fan suction side is at atmospheric conditions, or,

$$
\operatorname{FanSP}=p_{f, e}
$$

Eq. 76

As can be seen from Figure 37 at the fan outlet the pressure is,

$$
p_{f, e}=\Delta p_{f a n}
$$

and according to Eq. 76,

$$
F a n S P=\Delta p_{f a n}
$$

Eq. 78

from Eq. 69 and Eq. 72,

$$
-53.392 \dot{\forall}^{2}+334.34 \dot{\forall}+1945.7=23.05 \dot{\forall}^{2}
$$

Eq. 79

In other words, this is the point where the fan curve intersects the system curve (as can be seen from Figure 38), solving for $\dot{\forall}$, and inserting this value into Eq. 69 or Eq. 72 it is obtained that,

$$
\dot{\forall}_{\max }=7.685 \frac{\mathrm{m}^{3}}{\mathrm{~s}}
$$

and,

$$
F a n S P_{\text {max }}=1361 P a
$$

these values define the fan working point at maximum power (max fan speed). With these values, the maximum velocity at the test section is determined by,

$$
U_{0, \max }=\frac{\dot{\forall}}{A_{0}}
$$


using the numerical values yields,

$$
U_{0, \max }=34.7 \frac{\mathrm{m}}{\mathrm{s}}
$$

Figure 39 shows the static and total pressure (gage pressure) distribution along the wind tunnel (as was shown in Figure 37) using this velocity. As can be seen there is no pressure regain in the diffuser (as was assumed). The larger localized pressure loss occurs at the screen located at the fan exit. In all the calculations standard air was assumed.

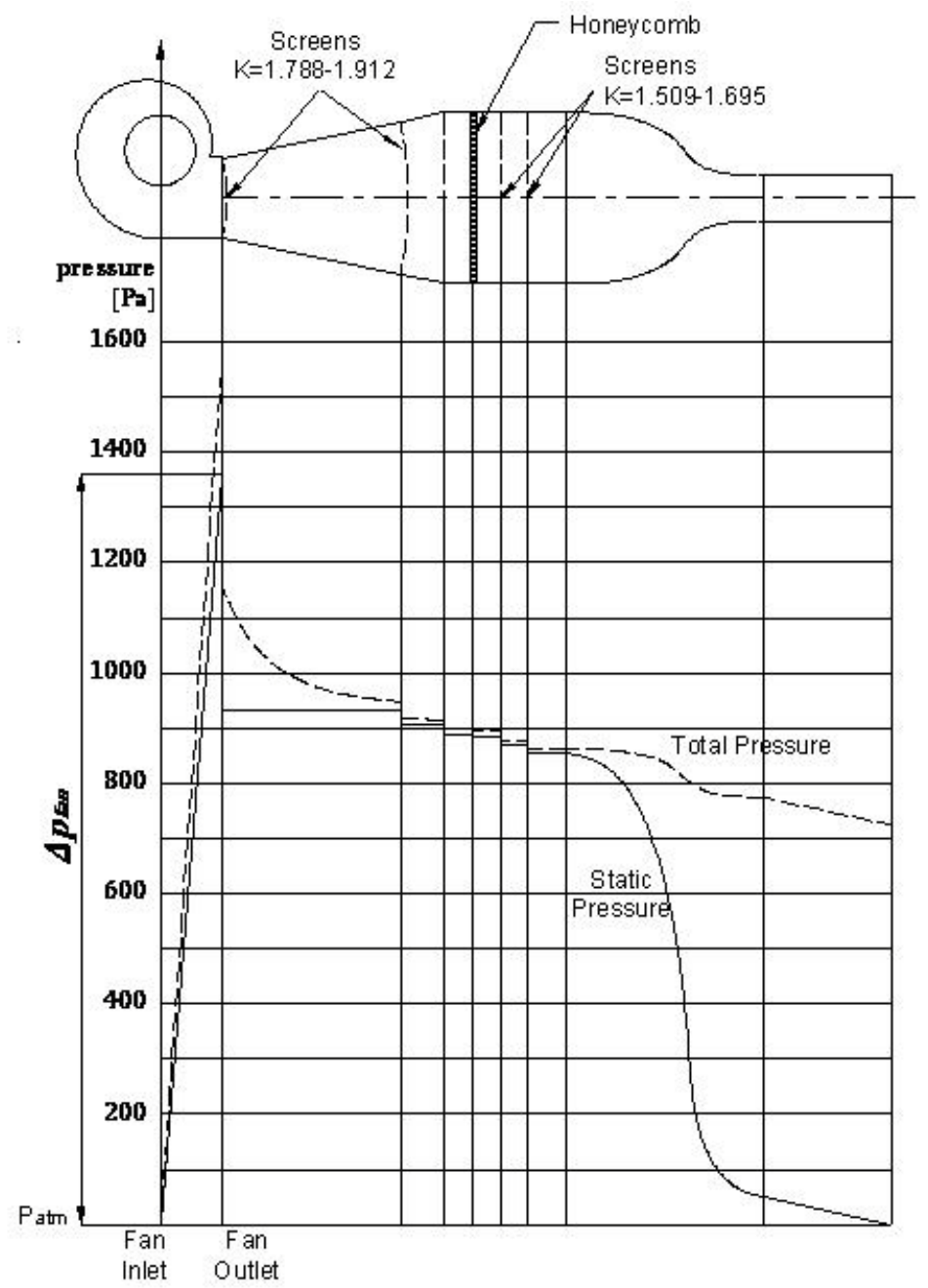

Figure 39. Static and Total pressure distribution along the wind tunnel for a test section velocity of $34.7 \mathrm{~m} / \mathrm{s}$. Gage pressure is shown 
Figure 40 shows the power required for the fan to operate, which was obtained using the manufacturer data [Cincinnati Fan, 2008]. . According to this curve, the power required to obtain the maximum velocity at the test section is,

$$
\dot{W}=21.7 H P
$$

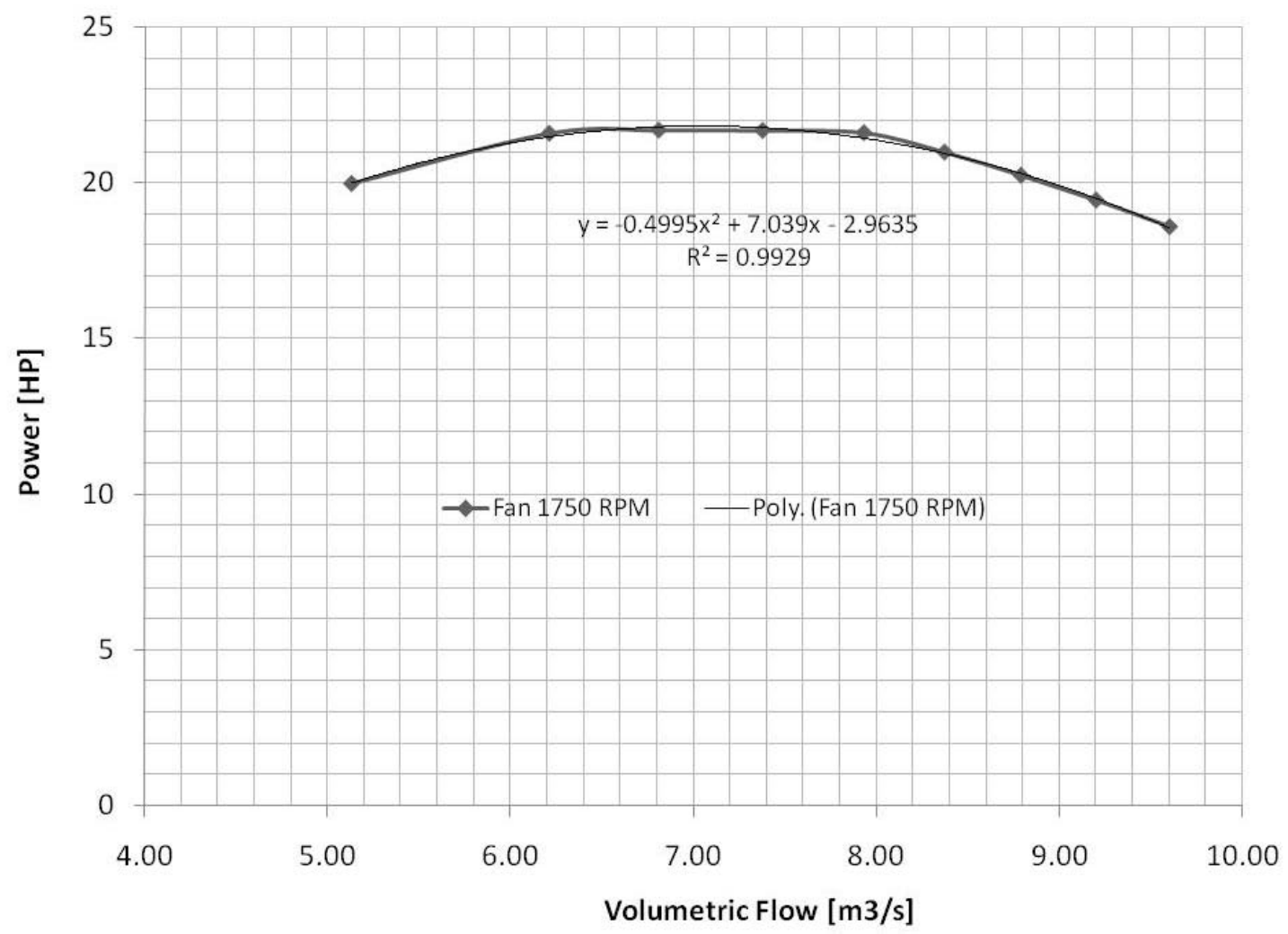

Figure 40. Fan power vs. volumetric flow curve (fan at max speed: 1750rpm) 


\section{GROUND SIMULATION DESIGN}

The wind tunnel that was constructed simulates the ground by using a moving belt. According to Figure 20 [Turner, 1966] the parameter $h$ over $b$ and the lift coefficient are helpful to decide what simulation technique can be used, and according to Table 1 for the Air Ray, the first parameter is between 0.077 and 0.308 and the lift coefficient is 3.5. Then according to Figure 20 [Turner, 1966] a moving belt is required.

It is recommended to have a boundary layer suction system at the beginning of the belt, as is shown in Figure 22 [Turner, 1967]. It has to be constructed with a smooth curvature. At that point the thickness of the boundary layer is greater than $20 \mathrm{~mm}$ (according to the simulations carried in Fluent). It is recommended that this suction system will be detachable in order to calibrate it by trial and error according to the actual value of the boundary layer thickness.

The useful length of the moving ground in the test section, $L_{G r}$, according to section 3.6 is three times the length of the model. Then using the values of Table 1 and the scaling factor (from section 11.1), it can be obtained that, this length is $516 \mathrm{~mm}$. Nevertheless, a longer length can be used, and this helps to reduce the boundary layer, in this case the belt length is going to be the full length of the exit duct, then,

$$
L_{G r}=1066 \mathrm{~mm}
$$

The actual length is shorter since one of the rollers has to be inside of the test section.

To simulate the updrafts, the belt velocity has to be lower than that of the air velocity. 


\section{GENERAL LAYOUT}

Now that the dimensions of all the components have been determined, a general layout of the wind tunnel can be defined. Figure 41 through Figure 45 show this general layout. Figure 41 shows the main components of the wind tunnel. Figure 42 shows the main dimensions of the wind tunnel when it is tilted $50^{\circ}$ and also shows the tilting mechanism. Figure 43 shows the main dimensions of the wind tunnel. Figure 44 is a exploded view of aerodynamic components, showing the ducts and the flow conditioners. Figure 45 shows the components of the wide angle diffuser. The characteristics of this layout are discussed below.

The diffuser is formed by three independent components that are bolted together, and between them are placed the screens. The Settling Chamber is formed by five components, three screens, a honeycomb and a spacer. Each screen is installed in an independent frame, along the honeycomb. This facilitates the installation of more screens, if required. The screen frames of the diffuser are curved to force the required shape of the screens.

The contraction is a single component and is bolted to the contraction spacer and to the test section.

As can be seen the test section is detachable, this is intended to allow the installation, if required, of a different test section, for other studies, for example 2D flows, HVAC components, calibration of flow devices, etc.

The test section does not have a floor, in order to allow the moving belt to be placed, which is an independent unit formed by the rolls, tensor system, motor, and drive system. This unit is supported by the component Adjustable Table, which can be displaced axially with respect to the Moving Structure, in case more screens would be needed.

The tilting mechanism can be activated by a linear actuator (hydraulic or electric). Since this is not the objective of this study it will not be detailed here. Nevertheless it is important that the pivot point of the wind tunnel be as close as possible to the center of mass of the movable part of the wind tunnel. Since the fan is the heaviest component, and the ducts are generally constructed in wood 
or metal sheets (which are lighter) the fulcrum will be close to the fan, as can be see in Figure 43. The weight of the movable part (fan, ducts, structures, ground simulator) of the wind tunnel is approximately $1140 \mathrm{Kg}$, and the distance from the fulcrum to the mass center is $1336 \mathrm{~mm}$.

Finally as can be seen (Figure 42) the total height of this layout when tilted will be less than six meters, which is not a problem since the hangar height is nine meters.

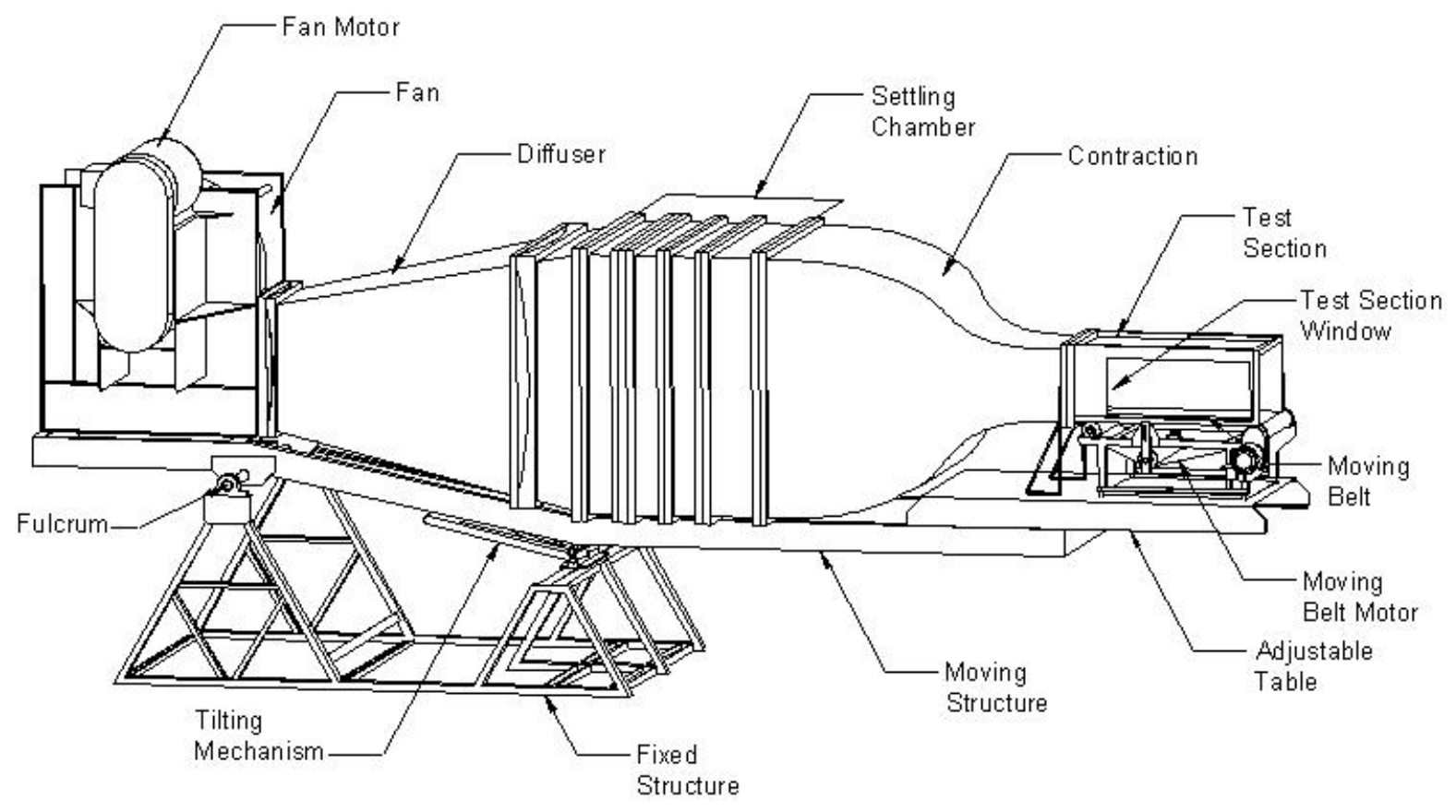

Figure 41. General Layout of the wind tunnel and its main components, horizontal position 


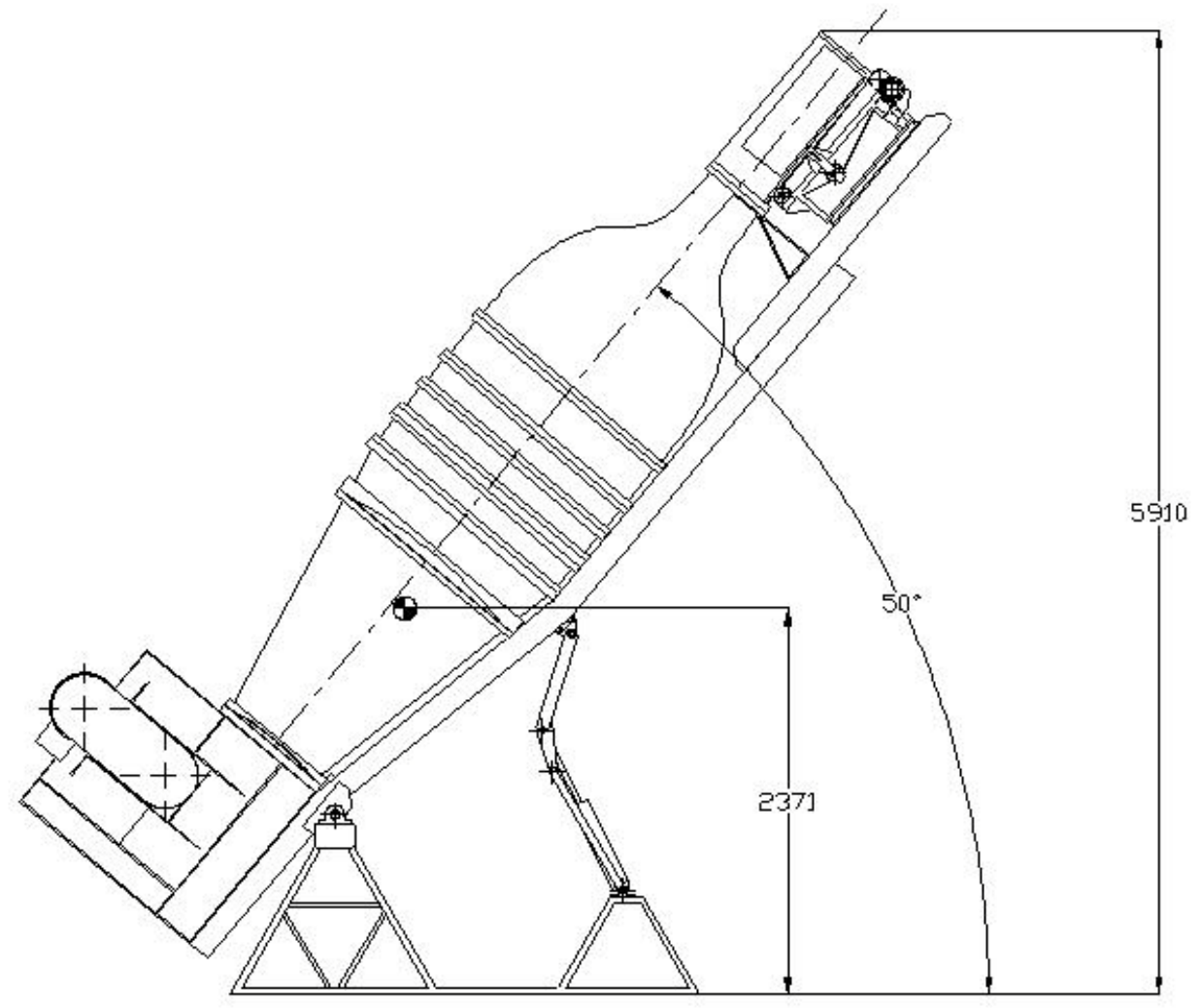

Figure 42. General Layout of the wind tunnel and its main components, tilted $50^{\circ}$ 

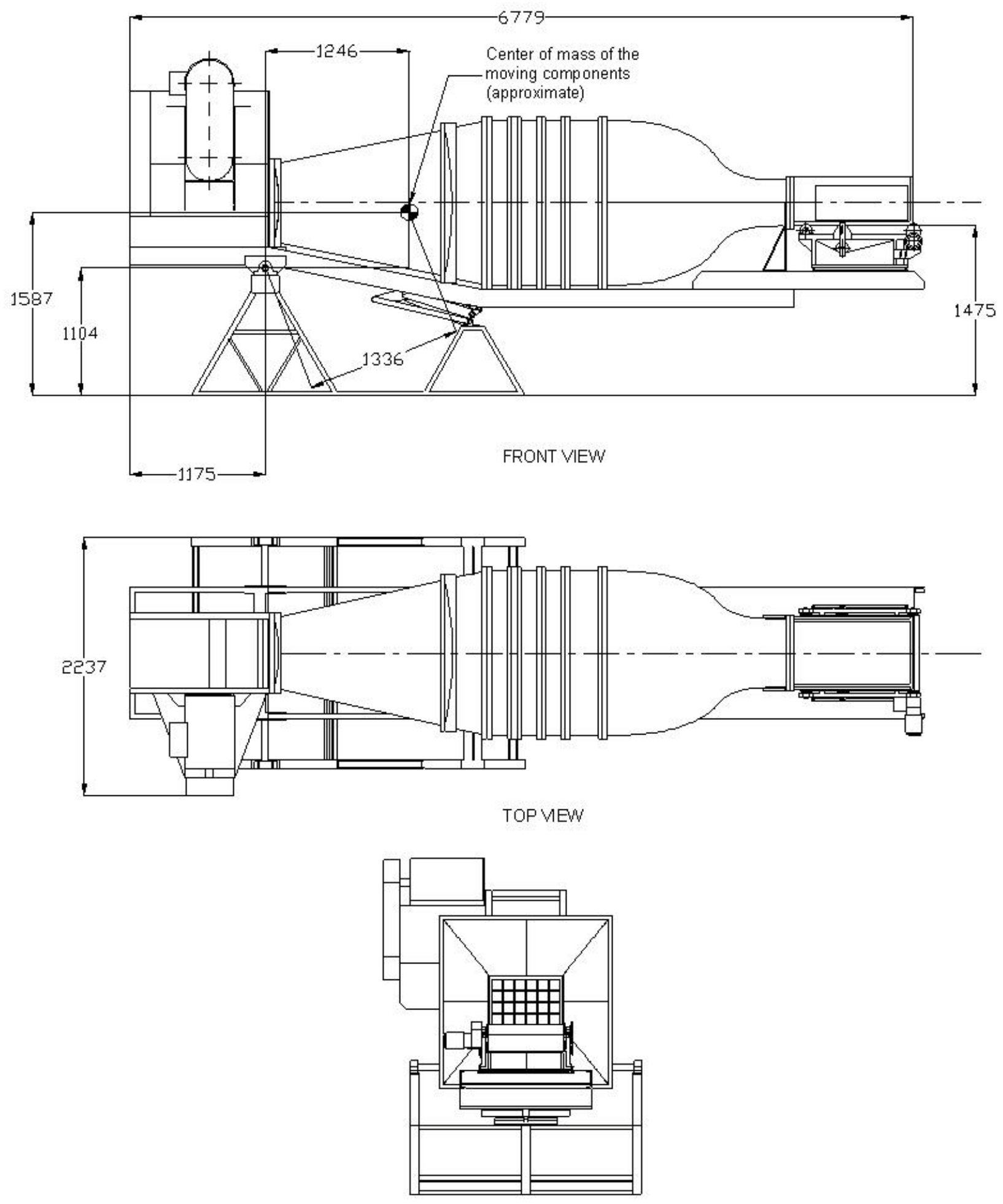

RIGHT MEW

Figure 43. Wind Tunnel Layout main dimensions, horizontal position 


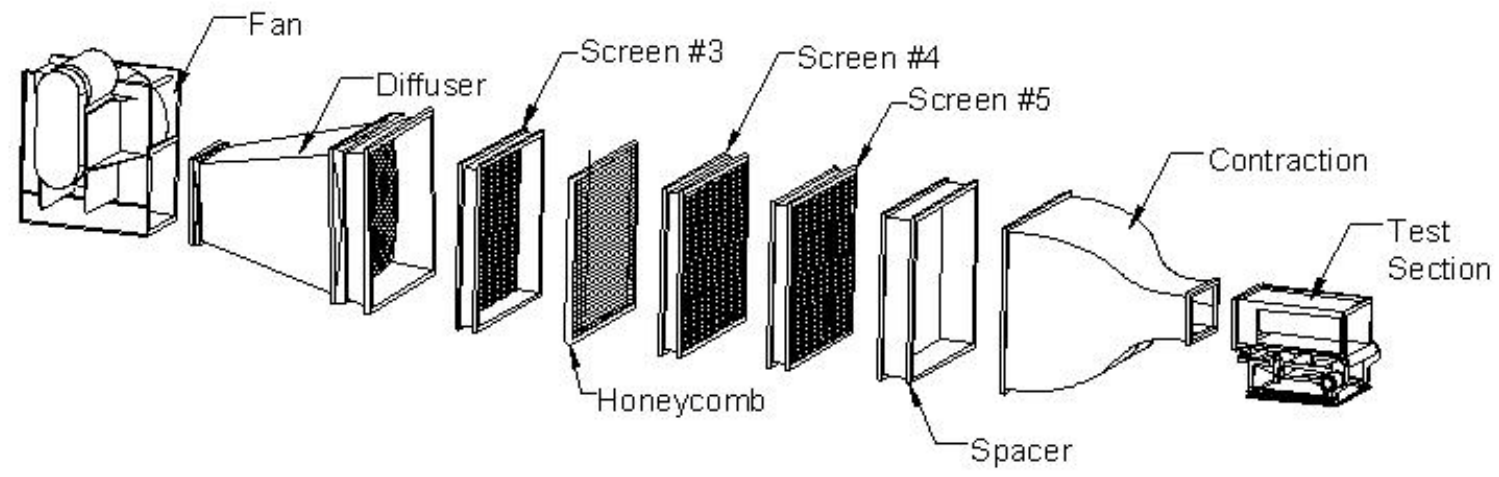

Figure 44. Wind Tunnel Layout fan and ducts, exploded view. The diffuser is detailed in Figure 45.

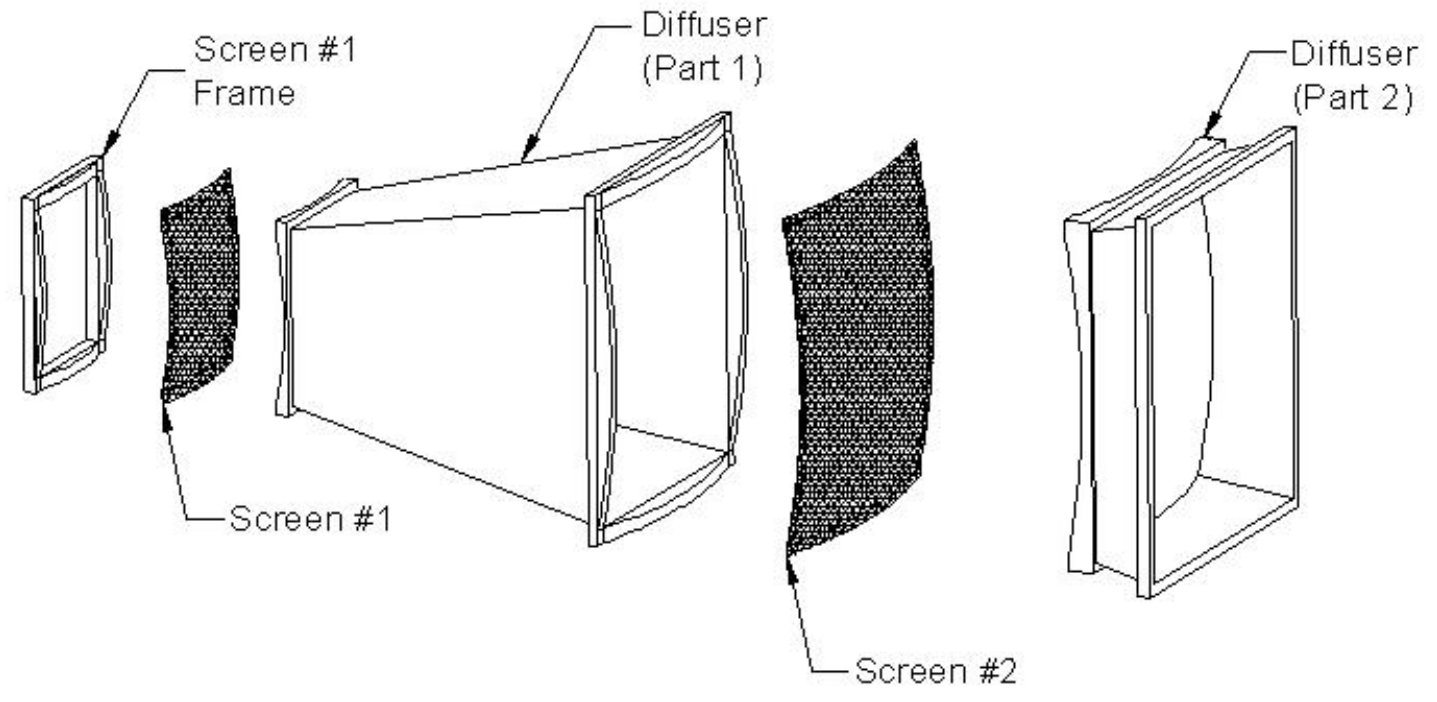

Figure 45. Diffuser. Exploded view 


\section{MODEL SIZE}

The size of the model has to be determined according to the scaling parameters (Froude number for this case) and at the same time has to facilitate the construction of the model and minimize the correction factors and its calculations. A very small model will be harder to construct and will require close tolerances and the model and prototype Reynolds number will differ considerably, leading to different flow patters. On the contrary a very large model is easier to construct but the data obtained may require several corrections due to the effects of the test section boundaries.

In the next section the scaling factor will be obtained according to these criteria.

\subsection{SCALING FACTOR}

If the recommend value of $5 \%$ for the blockage area is used, then,

$$
\frac{A_{m}}{A_{0}}=0.05,
$$

Eq. 81

where $A_{m}$ is the model frontal area. Using the value for $A_{0}$, yields,

$$
A_{m} \cong 11059 \mathrm{~mm}^{2} \text {. }
$$

The scaling factor $\lambda$ is defined as,

$$
\lambda=\frac{L_{p}}{L_{m}},
$$


where $L_{p}$ and $L_{m}$ are, respectively, the prototype and model characteristic lengths. This factor can be related with the model and prototypes characteristic areas (frontal area in this case) as,

$$
\lambda=\sqrt{\frac{A_{p}}{A_{m}}},
$$

from Table 1 the frontal area of the prototype, $A_{p}$, is $2.22 \mathrm{~m}^{2}$, then

$$
\lambda=\sqrt{\frac{2220000}{11059}} \cong 14.2,
$$

with this value the span, $W_{m}$, of the model will be (using the data form Table 1 ),

$$
W_{m}=\frac{W_{p}}{\lambda}=\frac{3960}{14.2} \cong 279 \mathrm{~mm}
$$

the relation between this value and the test section width is,

$$
\frac{W_{m}}{W_{0}}=\frac{279}{576} \cong 0.48
$$

which is less than the maximum recommended value of 0.8 (see section 3.5.2). Then a scaling factor of 14.2 can be used. As can be seen, the values of the blockage area and the scaling factor for those models showed on Table 4 are not far from the values of this case.

With this value for the scaling factor, the maximum test can be obtained using the fact that the Froude number for both, model and prototype has to be equal. Then, 


$$
\frac{U_{m}}{U_{p}}=\sqrt{\frac{L_{m}}{L_{p}}}
$$

or,

$$
\frac{U_{m}}{U_{p}}=\sqrt{\frac{1}{\lambda^{\prime}}}
$$

Eq. 86

using the value for the scaling factor yields,

$$
\frac{U_{m}}{U_{p}}=0.265
$$

Eq. 87

the value for the maximum velocity of the prototype is obtained from Table 1 , then,

$$
U_{m, \max }=6.5 \mathrm{~m} / \mathrm{s}
$$

this is the maximum required velocity at the test section, as can be seen it is smaller than the maximum obtainable velocity according to the fan maximum rpm (see section 8.2). With this velocity the required flow is,

$$
\dot{\forall}_{\max \text { max }}=1.438 \frac{\mathrm{m}^{3}}{\mathrm{~s}},
$$

and the fan speed (rpm) corresponding to this flow can be found using one the fan laws [American Conference of Governmental Industrial Hygienists, 1976],

$$
\frac{\dot{\forall}_{m, \max }}{\dot{\forall}_{\max }}=\frac{R P M_{m, \max }}{R P M_{\max }}
$$

inserting the values of $\dot{\forall}_{\text {m,max }}, \dot{\forall}_{\text {max }}$ and $R P M_{\text {max }}$, which is 1750rpm, and solving for $R P M_{m, \max }$, it is obtained, 


$$
R P M_{m, \max }=327 \mathrm{rpm},
$$

this is approximately the fan speed needed to have the required speed at the test section.

Another important parameter that can now be determined is the model Reynolds number, this is shown in Table 14, which also indicates the prototype Reynolds numbers, both of them based on the vehicle length. The ratio between these Reynolds numbers is about 53 , due to this difference the flow patterns in the model and the prototype will be different. But as was stated in section 3.3, the effect of this difference can be reduced if the transition points are conserved.

Table 14. Model and prototype Reynolds number, base on the vehicle length

\begin{tabular}{|c|c|}
\hline $\begin{array}{c}\text { Prototype Reynolds } \\
\text { number }\end{array}$ & $\begin{array}{c}\text { Model Reynolds } \\
\text { number }\end{array}$ \\
\hline $3.97 \times 10^{6}$ & $7.45 \times 10^{4}$ \\
\hline
\end{tabular}

\subsection{TOLERANCE ANALISIS}

The problem of having a very small model beside the problem associated with the required flow similitude (i.e. matching several scaling number as for example Reynolds number and Froude number) is that the required construction precision increases in order to have a model that is geometrically similar to the prototype. The tolerances from the model are determined below, using the procedure used by Barlow, et al. (1999).

The nominal maximum thickness of the prototype wing is $300 \mathrm{~mm}$, and for the model will be $21.13 \mathrm{~mm}$ (using the obtained value for the scaling factor of 14.2). Now, if the dimensions of the model that is going to be constructed have to be between the 2\% (as suggested by Barlow, et al., (1999)), then this thickness has to be between $21.13 \pm 0.42 \mathrm{~mm}$ for the model. According to the general tolerances for metal working, this value corresponds to a tolerance class of "Coarse". The model could be constructed without any problem using for example a milling machine, but usually due to the required light weight 
requirements, metals cannot be used, thus wood, plastics, or composites should be used. Some of these materials are difficult to work with in order to obtain close tolerances, but since this is not the case here, the model can be constructed with some care, for example using balsa.

\subsection{TEST AND MODEL RANGE VARIABLES}

Using the scaling factor value, the variation range of the test variables can be specified, as is shown in Table 15.

Table 15. Test variables range

\begin{tabular}{|l|c|}
\hline Air velocity & $1.6-6.5 \mathrm{~m} / \mathrm{s})$ \\
\hline Slope Angle & $0-50^{\circ}$ \\
\hline Wind Velocity (draft) & $0-1.1 \mathrm{~m} / \mathrm{s}$ \\
\hline Flap angle & $6^{\circ}-25^{\circ}$ \\
\hline Model Total Weight & $39-65$ grams \\
\hline
\end{tabular}




\section{CONCLUSIONS}

A low speed small wind tunnel was designed following the method proposed by Mehta (1977). It is an open blower wind tunnel with a tilting capability of $50^{\circ}$ and with a moving belt to simulate the ground. It has a rectangular test section of $384 \mathrm{~mm}$ (height) by $576 \mathrm{~mm}$ (width), and the maximum obtainable velocity at the test section is approximately $35 \mathrm{~m} / \mathrm{s}$.

To simulate the operation of the Air-Ray prototype a scaling factor of 14.2 with a blockage area of $5 \%$ should be used, this leads to a model span of $279 \mathrm{~mm}$ and a required maximum test velocity of $6.5 \mathrm{~m} / \mathrm{s}$.

The fan of the previous wind tunnel can be used in this new design.

The obtained values for the scaling factor and blockage area are between the normal values used by several references. 


\section{RECOMMENDATIONS}

After the construction, perform a survey of the flow conditions at the test section. Particularly determine the velocity profile and turbulence intensity at the test section and verify it meets the recommended values.

If required the corners of some the wind tunnel components can be filled to avoid separation at these spots. If there is a considerable pressure drop at the test section these fillets can be tapered, in such a way that the inlet area will be smaller than the outlet area. This is done by trial and error until and adequate behavior of the flow at the test section is achieved.

If required, the flow uniformity at the test section can be increased by adding more screens.

Follow the recommendations given by Mehta (1977) related to operating conditions, construction materials and construction techniques. 


\section{REFERENCES}

Ahmed, M.R., S. Habeeb Ali, G.M. Imran, and S.D. Sharma. "Experimental Investigation of the Flowfield of a Symmetrical Airfoil in Ground Effect." Orlando, Florida: 21st AIAA Applied Aerodynamics Conference, 2003.

American Conference of Governmental Industrial Hygienists. Industrial Ventilation. 14th Edition. 1976.

Angle II, Gerald M. Air-Ray: Recreational Downhill Glider. Internal Report, West Virginia University, Morgantown, WV: Center for Industrial Research Applications, 2007.

Angle II, Gerald M. Recalculation of Air Ray Surface Area Requirements. Internal Report, West Virginia University, Morgantown, WV: Centerfor Industrial Research Applications, 2006.

Angle II, Gerald M. Stall Speed Determination for Air Ray. Internal Report, Morgantown, WV: Center for Industrial Research Applications, 2008.

Barlow, J.B., W.H. Jr Rae, and A. Pope. Low-speed Wind Tunnel Testing. Third Edition. Wiley, 1999.

Bell, J. H., and R.D. Mehta. "Contraction Design for Small Low-Speed Wind Tunnels." Joint Institute for Aeronautics and Acoustics, 1988.

Bell, J.H., and R.D. Mehta. "Boundary-Layer Predictions for Small Low-Speed Contractions." AIAA Journal 27 (1989): 372-374.

Bradshaw, P. Experimental Fluid Mechanics. 1964.

Brown, W.S. Wind Tunnel Corrections on Ground Effect. London: His Majesty's Stationery Office, 1939.

Chawla, M.D., L.C. Edwards, and M.E. Franke. "Wind-Tunnel Investigation of Wing-in-Ground Effects." Journal of Aircraft 27 (1990): 289-293.

Cincinnati Fan. "SQAF-905." 2008.

Downie, J.H., R. Jordinson, and F.H. Barnes. "On the Design of ThreeDimensional Wind Tunnel Contractions." Aeronautical Journal, 1984. 
Gibbings, J.C. "Incompressible Flow in Contracting Ducts." Aeronautical Journal, 1993: 230-246.

Heyson, H. H. "Rapid Estimation of Wind Tunnel Corrections with Applications to Wind Tunnel and Model Design." NASA, 1971.

Heyson, H.H. "Theoretical Study of Conditions Limiting VISTOL Testing in Wind Tunnels with Solid Floor." NASA, 1970.

Hubbell, Meagan L., Jonathan Kweder, John C. Ruth, Kenneth A. Williams, and James E. Smith. "Adjustable Slope, Moving Ground Plane Wind Tunnel." 26th AIAA Aerodynamic Measurement Technology and Ground Testing Conference. Seattle, Washington, 2008.

Lee, Pai-Hung, C. Edward Lan, and Vincent U. Muirhead. "Experimental Investigation of Dynamic Ground Effect." Journal of Aircraft 26, no. 6 (1989).

Mehta, R. D. "Turbulent Boundary Layer Perturbed by a Screen." AIAA Journal 23 (1985).

Mehta, R. D., and P. Bradshaw. "Design Rules for Small Low-Speed Wind Tunnels." Aero Journal (Royal Aeronautical Society) 73 (1979): 443.

Mehta, R.D. "The Aerodynamic Design of Blower Tunnels with Wide-Angle Diffusers." Prog Aerospace Sci 18 (1977): 59-120.

Mull, T. E. HVAC Principles and Applications Manual. New York: McGraw-Hill, 1997.

Paulson, John W. "Investigation of the Low Speed Flight Characteristics of a 1/15-Scale Model of the Convair XB-58 Airplane." NACA, 1957.

Pennycuick, C. J., Thomas Alerstam, and Anders Hedenström. "A New LowTurbulence Wind Tunnel for Bird Flight Experiments at Lund University, Sweden." The Journal of Experimental Biology, 1997: 1441-1449.

Pope, Alan, and John J. Harper. Low-Speed Wind Tunnel Testing. John Wiley \& Sons, 1966.

Recant, I.G. "Wind-Tunnel Investigation of Ground Effect on Wing with Flaps." NACA, 1939.

Schliestett, George Van. "Experimental Verification of Theodorsen's Theoretical Jet-Boundary Correction Factors." NACA, 1934. 
Seair Craft Inc. Experimental Aerodynamics \& Hydrodynamics. NetClimber Web Design. 2003. http://www.seaircraft.com/experimental.htm (accessed October 2008).

Su, Yao-xi. "Flow Analysis and Design of Three-Dimensional Wind Tunnel Contractions." AIAA Journal 29 (1991): 1912-1920.

Turner, T. R. "A Moving-Belt Ground Plane For Wind-Tunnel Ground Simulation And Results For Two Jet-Flap Configurations." NASA, 1967.

Turner, T. R. "Endless-Belt Technique for Ground Simulation." NASA, 1966.

Urquhart, S.R., S.A. Prince, and V. Khodagolian. "Aerodynamic Study of Reversed-Delta Wing Surface Craft in Ground Effect." Reno, Nevada: 44th AIAA Aerospace Sciences Meeting and Exhibit, 2006.

Wolowicz, Chester H., Jr, James S. Bowman, and William P. Gilbert. "Similitude Requirements and Scaling Relationships as Applied to Model Testing." NASA, 1979.

Zerihan, J., and Xin Zhang. "Aerodynamics of a Single Element Wing in Ground Effect." Journal of Aircraft 37 (2000). 


\section{APPENDIX A}

\section{FAN DIMENSIONS}




\section{图 DIMENSIONS and SPECIFICATIONS}

\section{Arrangement \#9, Belt Drive}

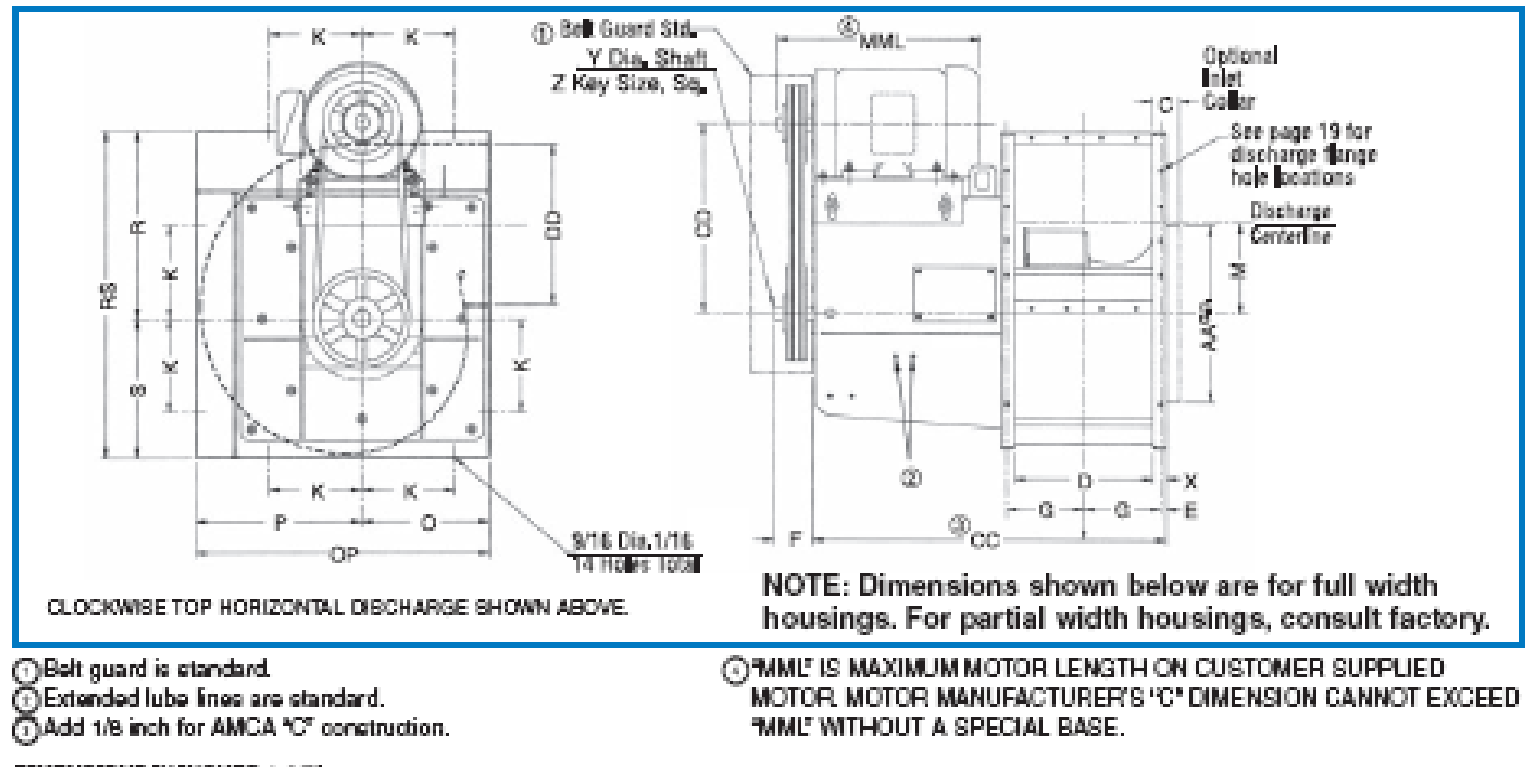

CAMENSBONS ININCHES $\div 1$ 1\%"

\begin{tabular}{|c|c|c|c|c|c|c|c|c|c|c|c|c|c|c|c|}
\hline \multirow[b]{2}{*}{ MODEL } & \multirow[b]{2}{*}{ C } & \multirow[b]{2}{*}{ D } & \multirow[b]{2}{*}{ E } & \multirow[b]{2}{*}{$\mathrm{F}$} & \multirow[b]{2}{*}{ G } & \multirow[b]{2}{*}{ K } & \multirow[b]{2}{*}{ M } & \multirow[b]{2}{*}{$\mathrm{O}$} & \multirow[b]{2}{*}{$P$} & \multirow[b]{2}{*}{ R } & \multirow[b]{2}{*}{$\mathrm{s}$} & \multirow[b]{2}{*}{$x$} & \multicolumn{3}{|c|}{ Y } \\
\hline & & & & & & & & & & & & & tII & GIJP & 5.1 \\
\hline $\operatorname{SCA}=-180^{\circ}$ & $1^{11 / 16}$ & $15 \%$ & $5 / 8$ & $41 / 2$ & $8^{12 / 16}$ & $10 \% / 8$ & $10^{13 / 32}$ & $14 \frac{1}{4}$ & $18^{1 / 2}$ & $20^{12 / 16}$ & $157 / 16$ & $11 / 2$ & $17 / 16$ & $111 / 6$ & - \\
\hline$S C A=202$ & $1^{11 / 14}$ & $17^{2 / 6}$ & $5 / 6$ & $41 / 2$ & $9^{2 / 14}$ & $113 / 4$ & $11^{13 / 32}$ & $151 / 2$ & $20^{2 / 3}$ & $22^{5 / 8}$ & 17 & $11 / 2$ & $111 / 48$ & $115 / 16$ & $2^{7 / 46}$ \\
\hline $\operatorname{scA}=220$ & $1^{11 / 14}$ & $19 \%$ & $5 / 6$ & $41 / 2$ & $10^{2 / 16}$ & $131 / 4$ & $12^{11 / 16}$ & 17 & $22^{2 / 4}$ & 25 & $187 / 8$ & $11 / 2$ & $111 / 46$ & $115 / 16$ & $2^{7 / 46}$ \\
\hline$S C A=249$ & $2^{1 / 4}$ & $215 / 16$ & $5 / 8$ & $41 / 2$ & $11^{17 / 32}$ & $142 / 4$ & $13^{21 / 20}$ & $18 \% / 2$ & $2413 / 16$ & $27^{\mathrm{J} / \mathrm{s}}$ & $203 / 4$ & $11 / 2$ & $1^{11 / 44}$ & $1^{15 / 46}$ & $2^{7 / 46}$ \\
\hline$S C A=270$ & $21 / 4$ & $231 / 2$ & $7 / k$ & $41 / 2$ & $12^{7 / 3}$ & $161 / 2$ & $15 \%$ & $20 \% / 4$ & $27^{1 / 4}$ & $30^{1 / 2}$ & $22^{7 / s}$ & 2 & $2^{3 / 16}$ & $2^{2 / 46}$ & $2^{7 / 46}$ \\
\hline$S C A=302$ & $2^{1 / 4}$ & $261 \%$ & $7 / k$ & $41 / 2$ & $14^{2 / 1}$ & $181 / 2$ & $17^{3} / 28$ & $22^{1 / 4}$ & $301 / 4$ & $33^{11 / 46}$ & $255 / 16$ & 2 & $2^{2} / 16$ & $2^{7 / 44}$ & $2^{11 / 16}$ \\
\hline
\end{tabular}

\begin{tabular}{|c|c|c|c|c|c|}
\hline \multirow{2}{*}{ Moof } & \multicolumn{3}{|c|}{2} & \multirow[b]{2}{*}{$A A$} & \multirow[b]{2}{*}{ DD } \\
\hline & ti! & $t 5$ & 5. & & \\
\hline SOA-182 & $3 / \mathrm{s}$ & $8 / 6$ & - & $20 \%$ & $17^{12 / 16}$ \\
\hline$S Q A=-202$ & $3 / \mathrm{s}$ & $1 / 2$ & $5 \%$ & $22 \%$ & $19^{7 / 16}$ \\
\hline $\operatorname{SOA}=220$ & $8 / 8$ & $1 / 2$ & $5 / 4$ & $24 \%$ & $21^{5 / 9}$ \\
\hline SDA $=240$ & $8 / \mathrm{s}$ & $1 / 2$ & $5 / 9$ & $27^{3 / 44}$ & $23^{12 / 16}$ \\
\hline$S 0 A=270$ & $1 / 2$ & $1 / 2$ & $5 \mathrm{se}$ & $30^{2 / 4}$ & $261 / 4$ \\
\hline SCA $=302$ & $1 / 2$ & $5 / 8$ & $5 / 4$ & $33 \%$ & $29^{2 / 16}$ \\
\hline \multicolumn{6}{|c|}{ Min_/Max, Motor Frame Sizes } \\
\hline \multirow{2}{*}{$\begin{array}{c}\text { Mope. } \\
\text { No. }\end{array}$} & \multirow{2}{*}{$\begin{array}{l}\text { FrAME } \\
\text { StDE }\end{array}$} & \multicolumn{4}{|c|}{\begin{tabular}{|c|} 
CLSSS \\
\end{tabular}} \\
\hline & & & ㅁ. & P & 口 \\
\hline \multirow[t]{2}{*}{$50,-185$} & MN. & & 56 & 56 & - \\
\hline & MAX. & & $154 T$ & $256 T$ & - \\
\hline \multirow[t]{2}{*}{$S D A=202$} & MW, & & 56 & $182 \mathrm{~T}$ & $218 \mathrm{~T}$ \\
\hline & $\operatorname{Max}$ & & $56 \mathrm{~T}$ & $286 \mathrm{~T}$ & $324 \mathrm{~T}$ \\
\hline \multirow[t]{2}{*}{$S C A=220$} & MN. & & 56 & 1327 & $213 T$ \\
\hline & MAX & & $56 \mathrm{~T}$ & $286 \mathrm{~T}$ & $324 \mathrm{~T}$ \\
\hline \multirow[t]{2}{*}{$s Q N-265$} & MN. & & 182T & $218 T$ & $218 T$ \\
\hline & $\operatorname{Max}$ & & $234 T$ & $324 T$ & $326 \mathrm{~T}$ \\
\hline \multirow[t]{2}{*}{$S D A=270$} & MIN. & & $13 \mathrm{~T}$ & $218 \mathrm{~T}$ & $254 \mathrm{~T}$ \\
\hline & $\operatorname{Max}$. & & $126 \mathrm{~T}$ & $324 T$ & $360 \mathrm{~T}$ \\
\hline \multirow[t]{2}{*}{$S C A=302$} & $\mathrm{M}$ W. & & $213 T$ & $213 T$ & $254 \mathrm{~T}$ \\
\hline & MAX & & $124 T$ & $326 \mathrm{~T}$ & 3655 \\
\hline
\end{tabular}




\section{APPENDIX B}

CFD GRID (CONTRACTION X=0.45) 


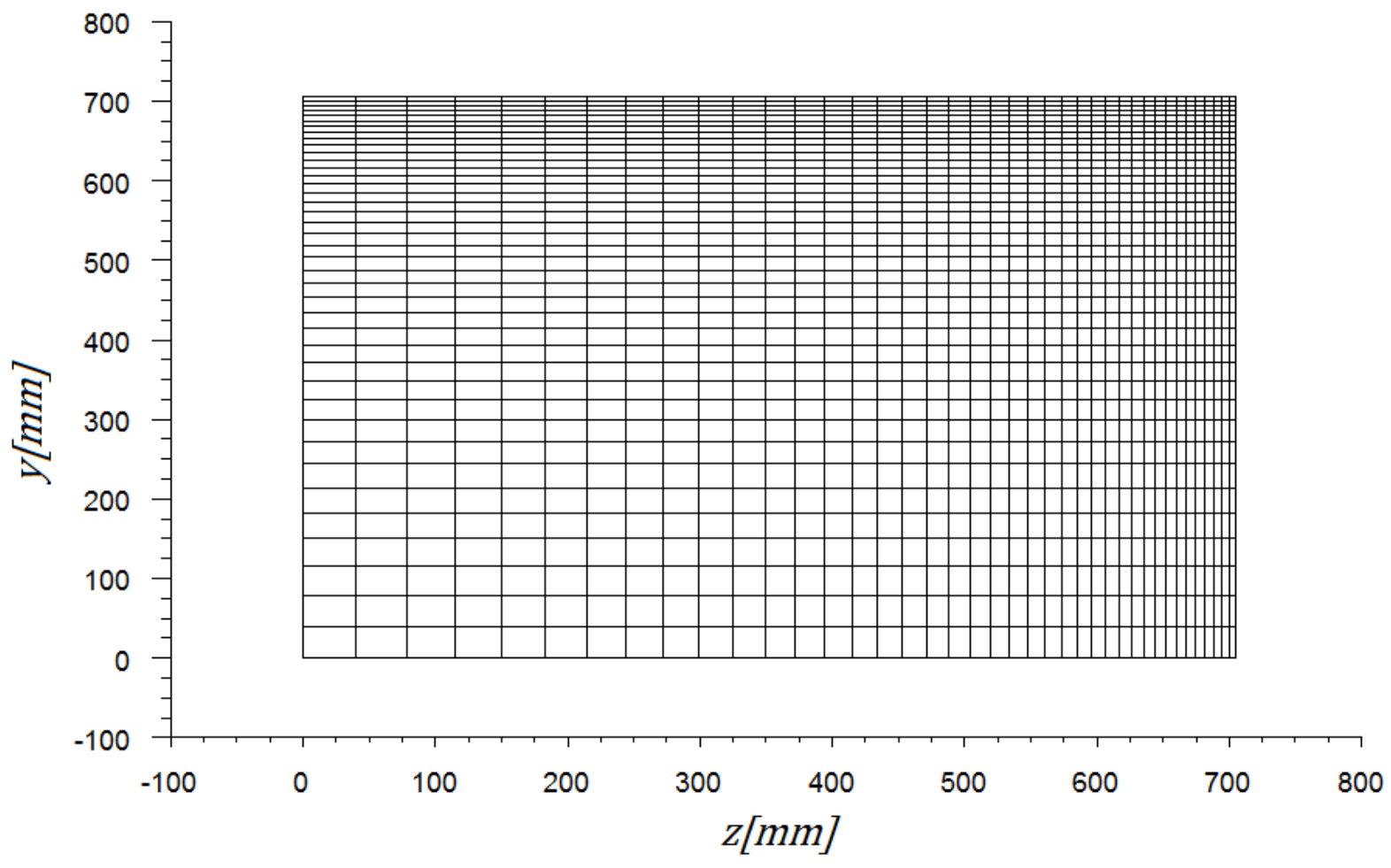

Figure AB1. Grid at the contraction inlet

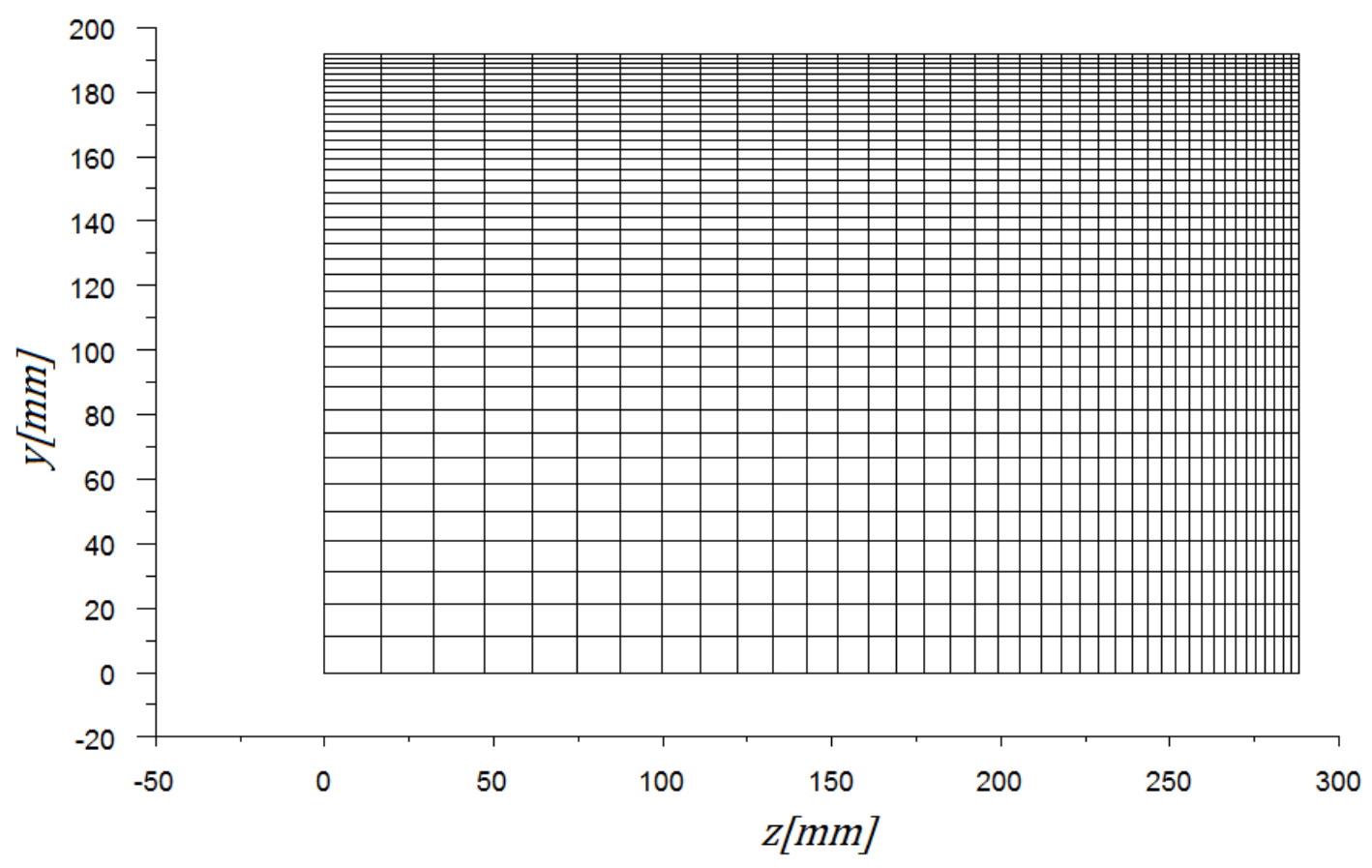

Figure AB2. Grid at the contraction exit 


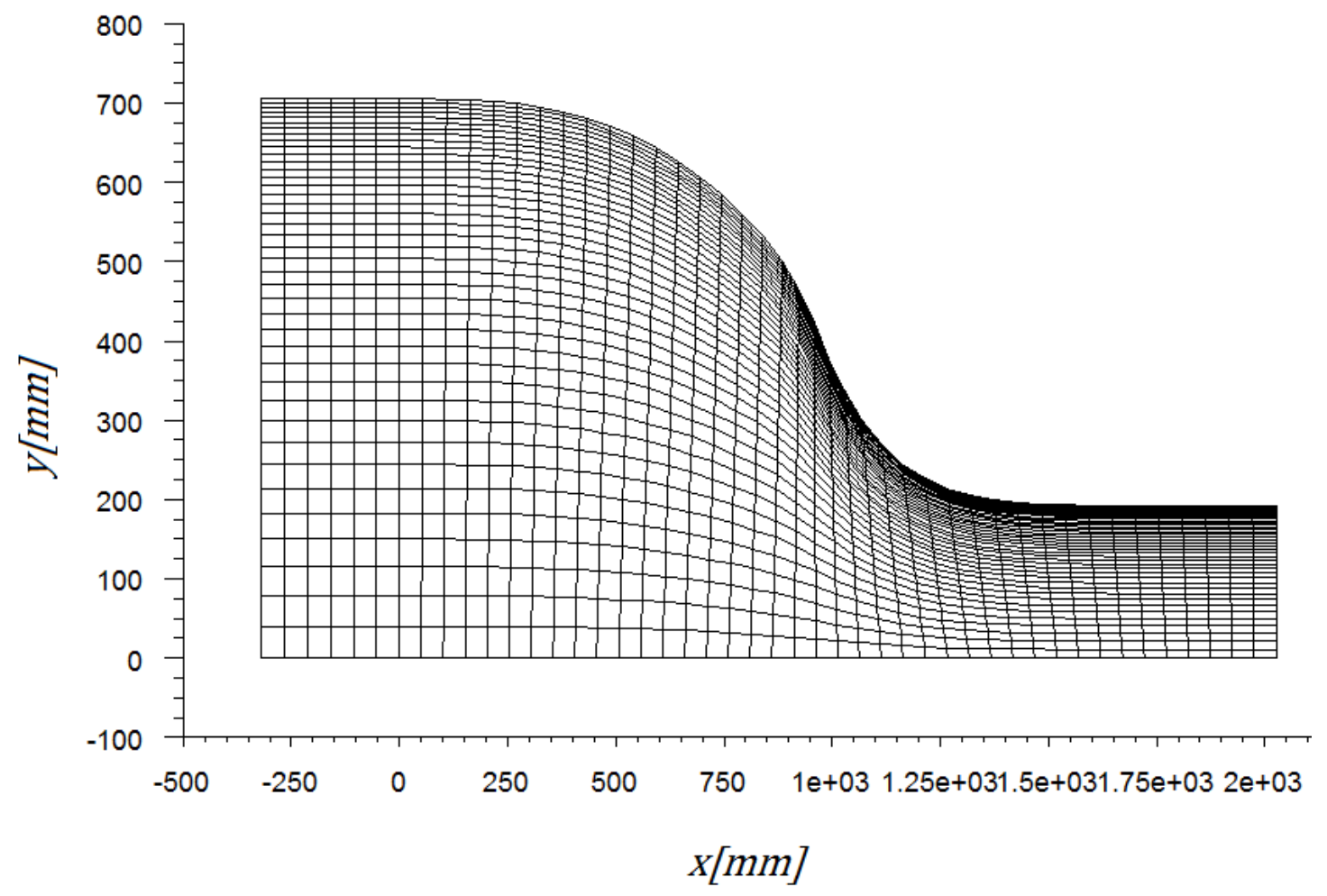

Figure $A B 3$. Grid at the $X Y$ plane

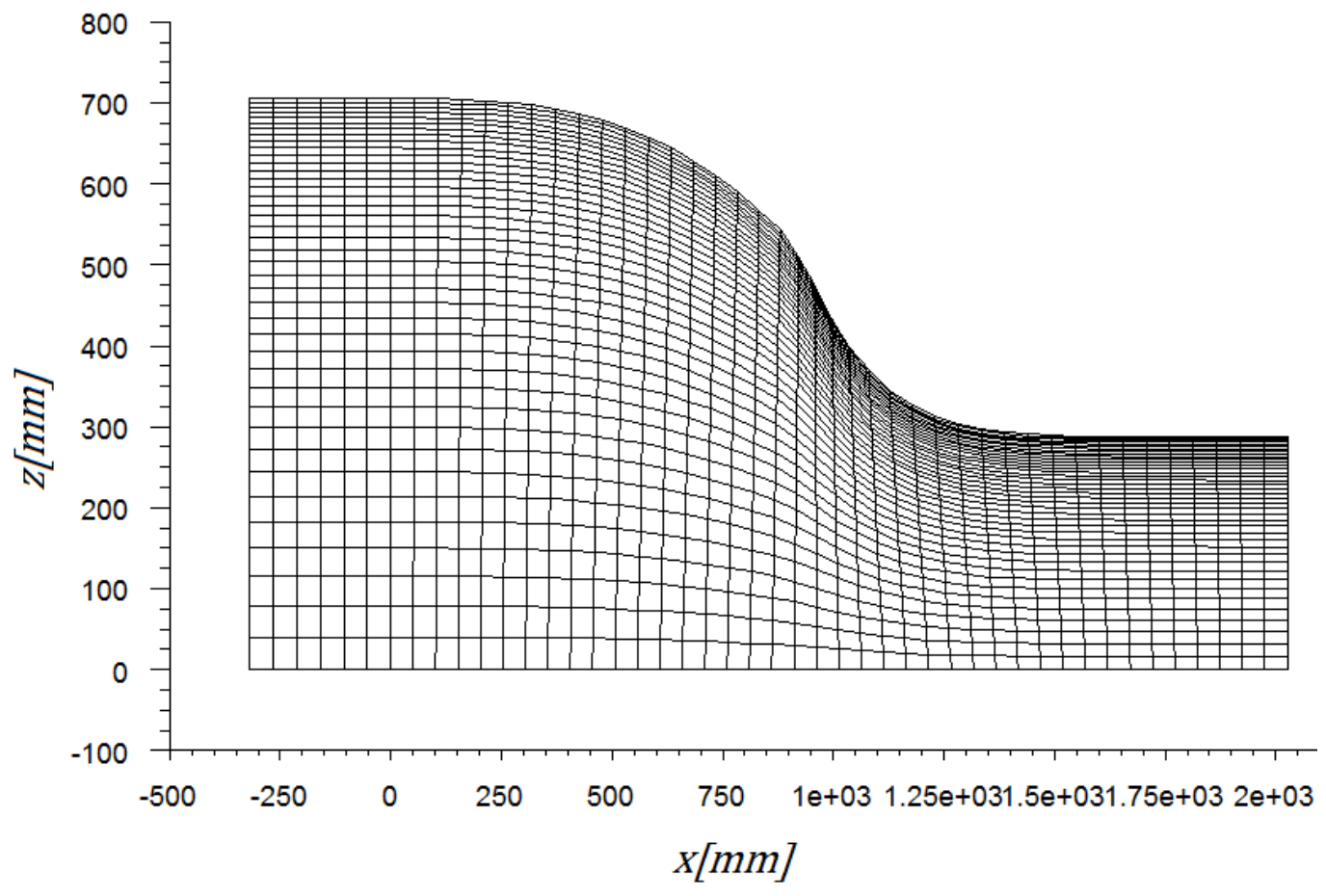

Figure AB4. Grid at the $X Z$ plane 


\section{APPENDIX C}

VELOCITIES PROFILES AT THE CONTRACTION EXIT

(Actual Length) 


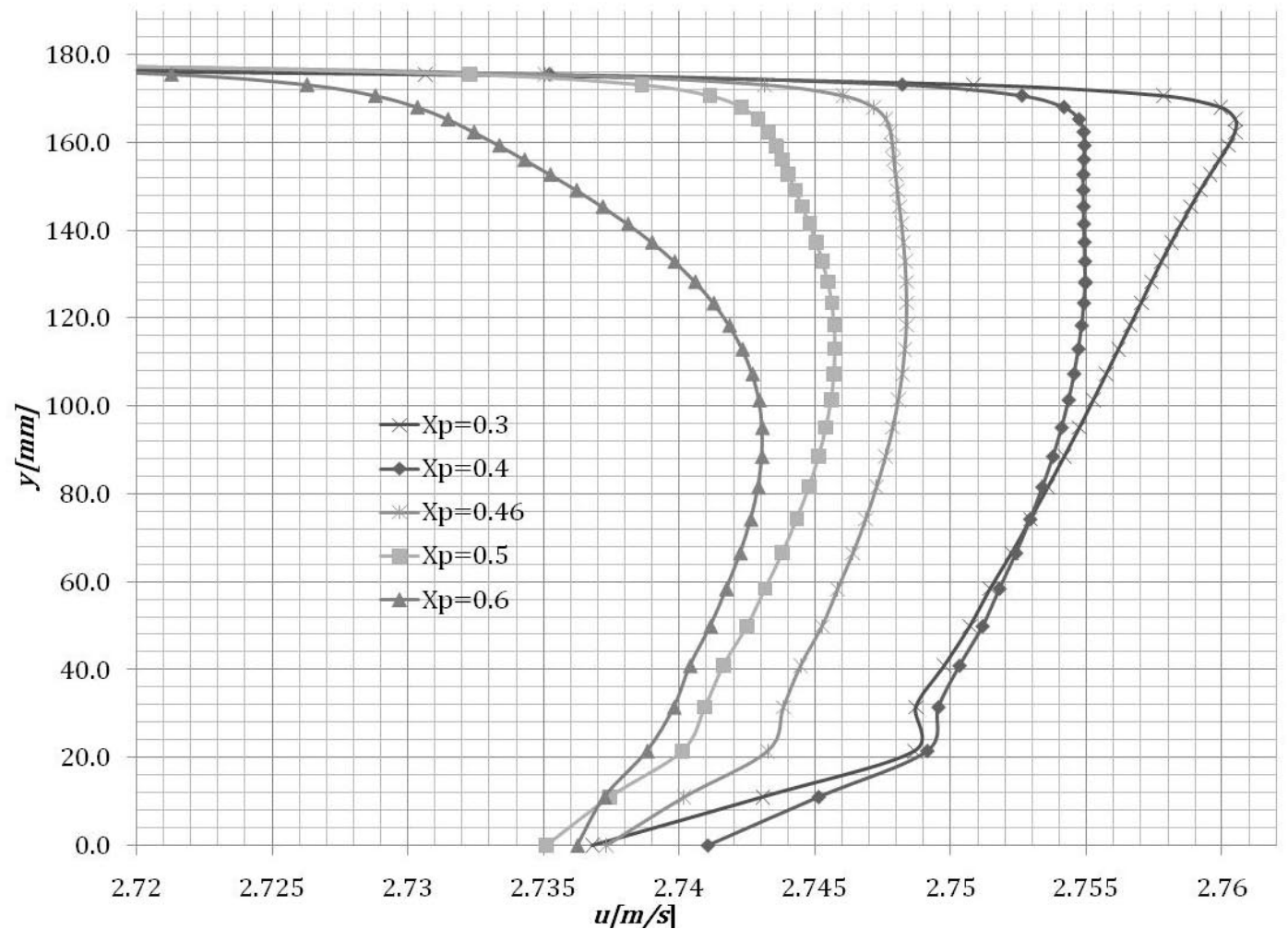

Figure AC1. Velocity profiles, for several Xp. XY plane, Inlet velocity: $0.3 \mathrm{~m} / \mathrm{s}$

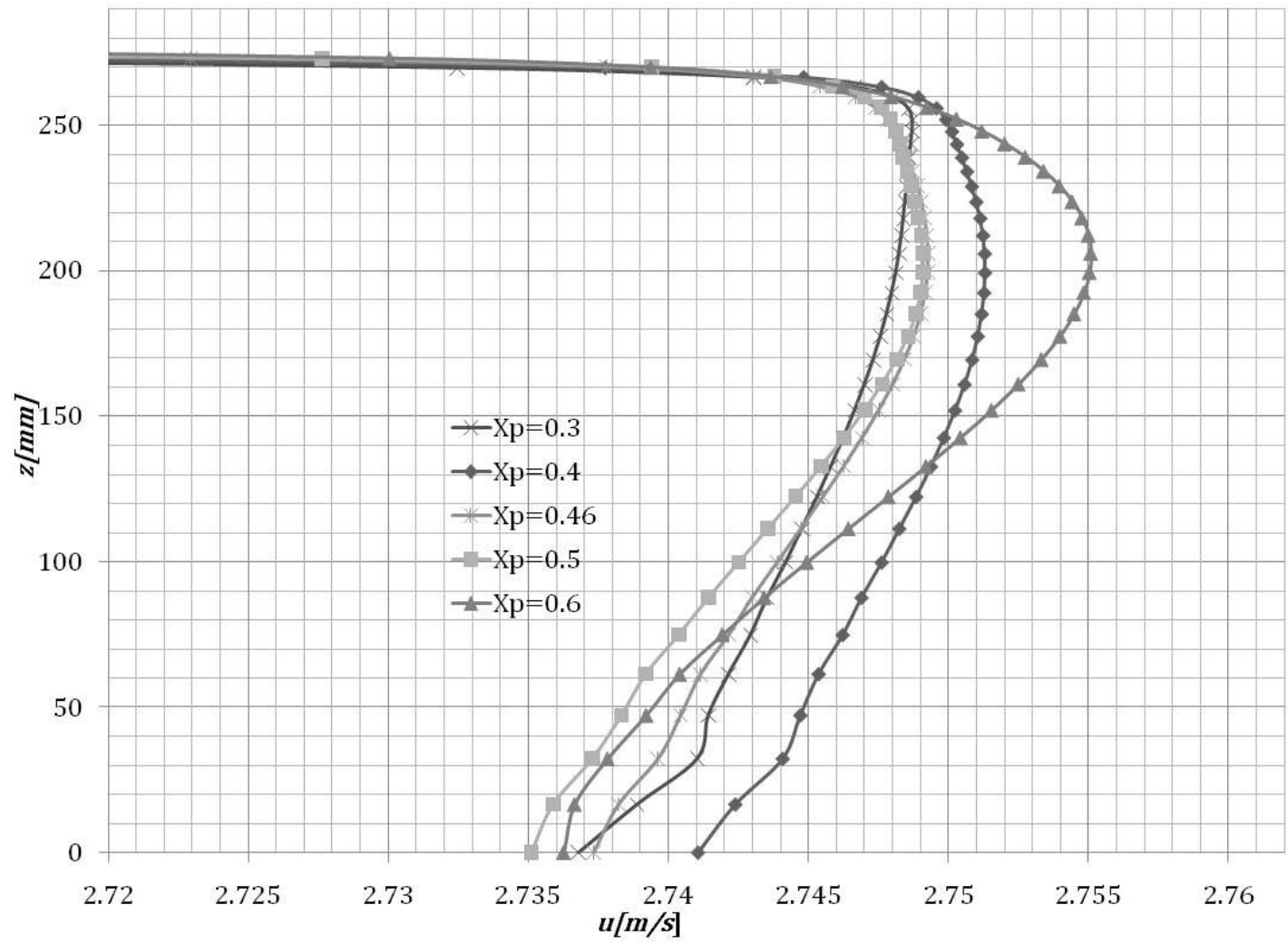

Figure AC2. Velocity profiles, for several Xp. XZ plane, Inlet velocity: $0.3 \mathrm{~m} / \mathrm{s}$ 


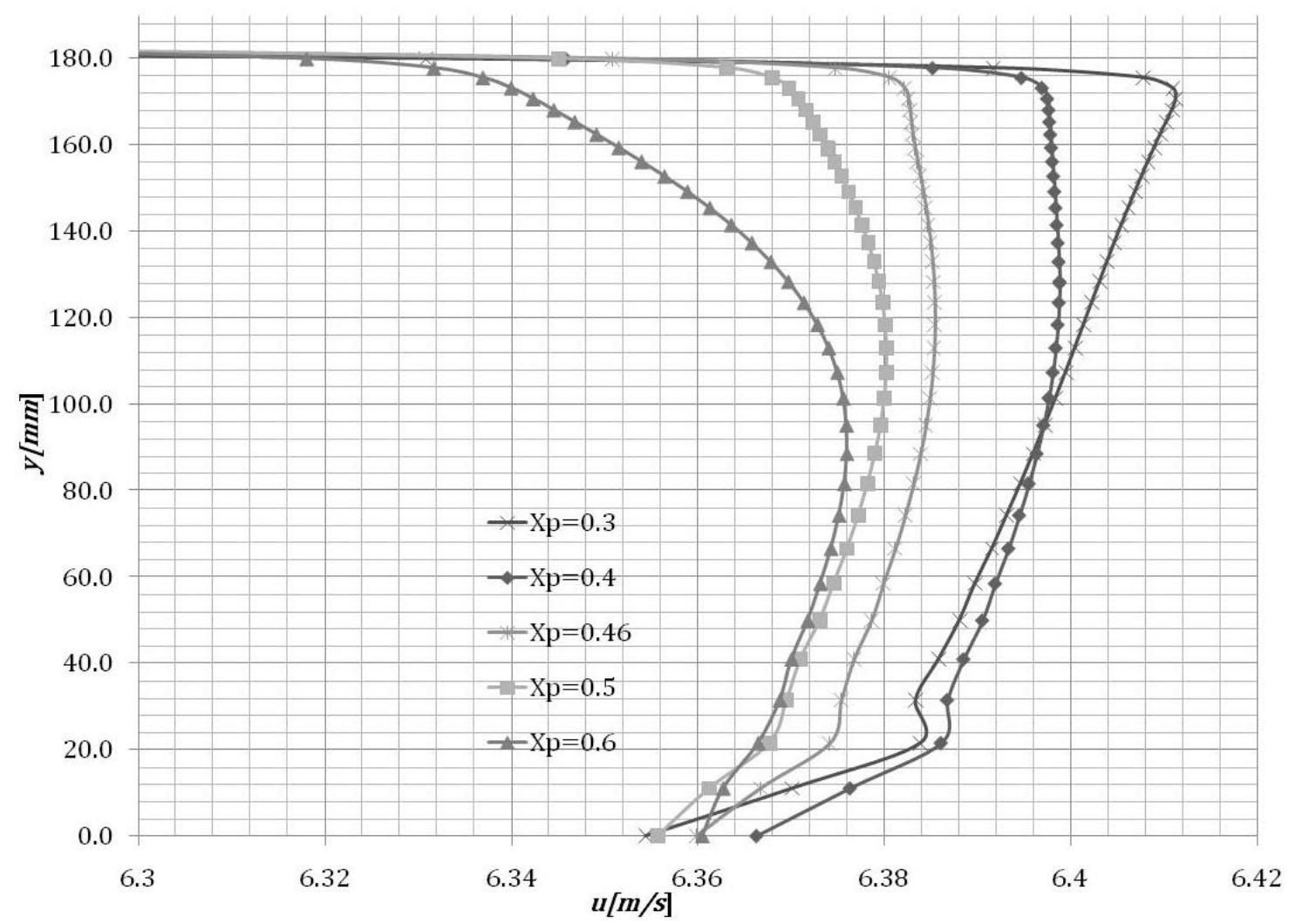

Figure AC3. Velocity profiles, for several Xp. XY plane, Inlet velocity: $0.7 \mathrm{~m} / \mathrm{s}$

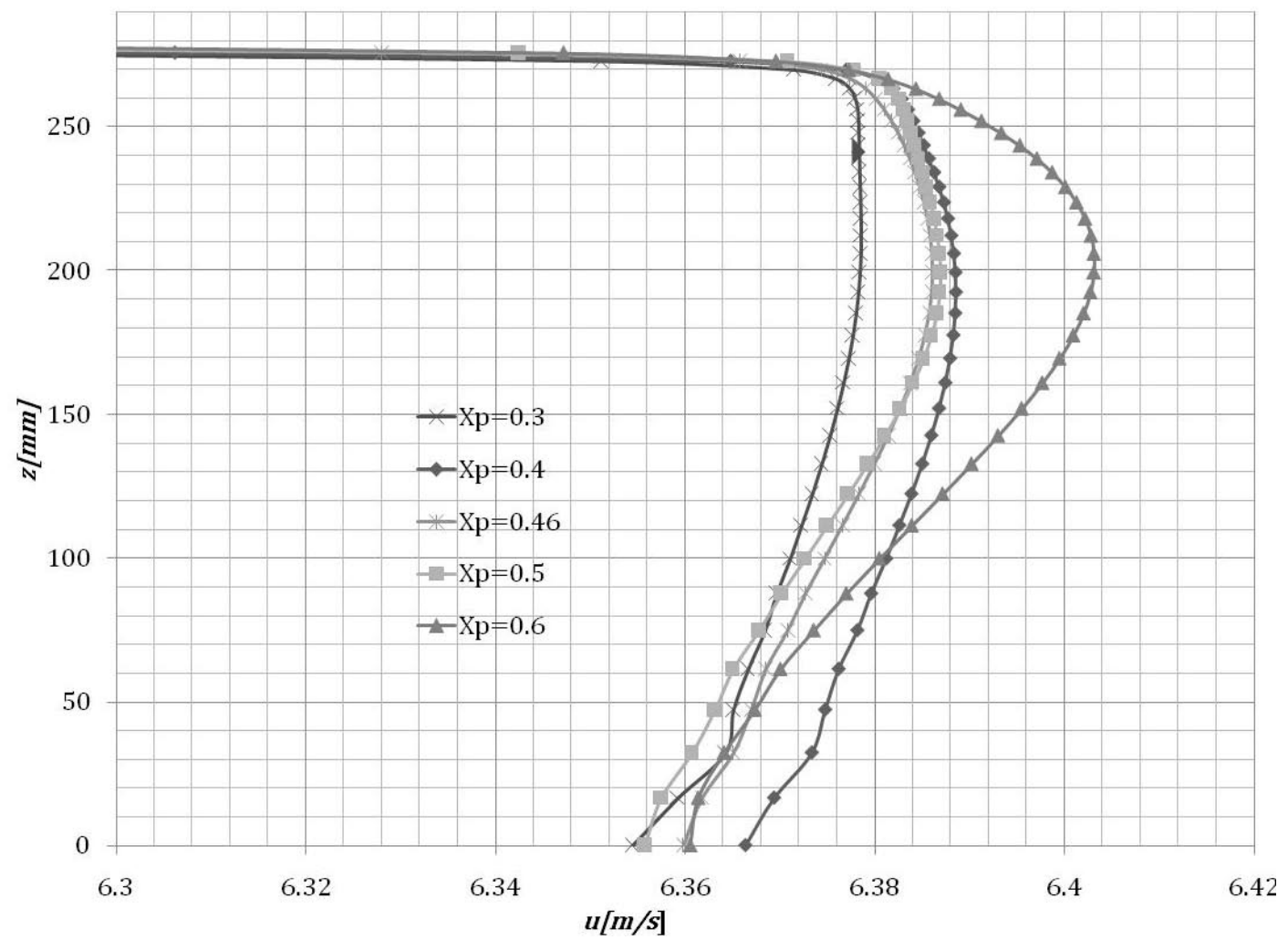

Figure AC4. Velocity profiles, for several Xp. XZ plane, Inlet velocity: $0.7 \mathrm{~m} / \mathrm{s}$ 120 


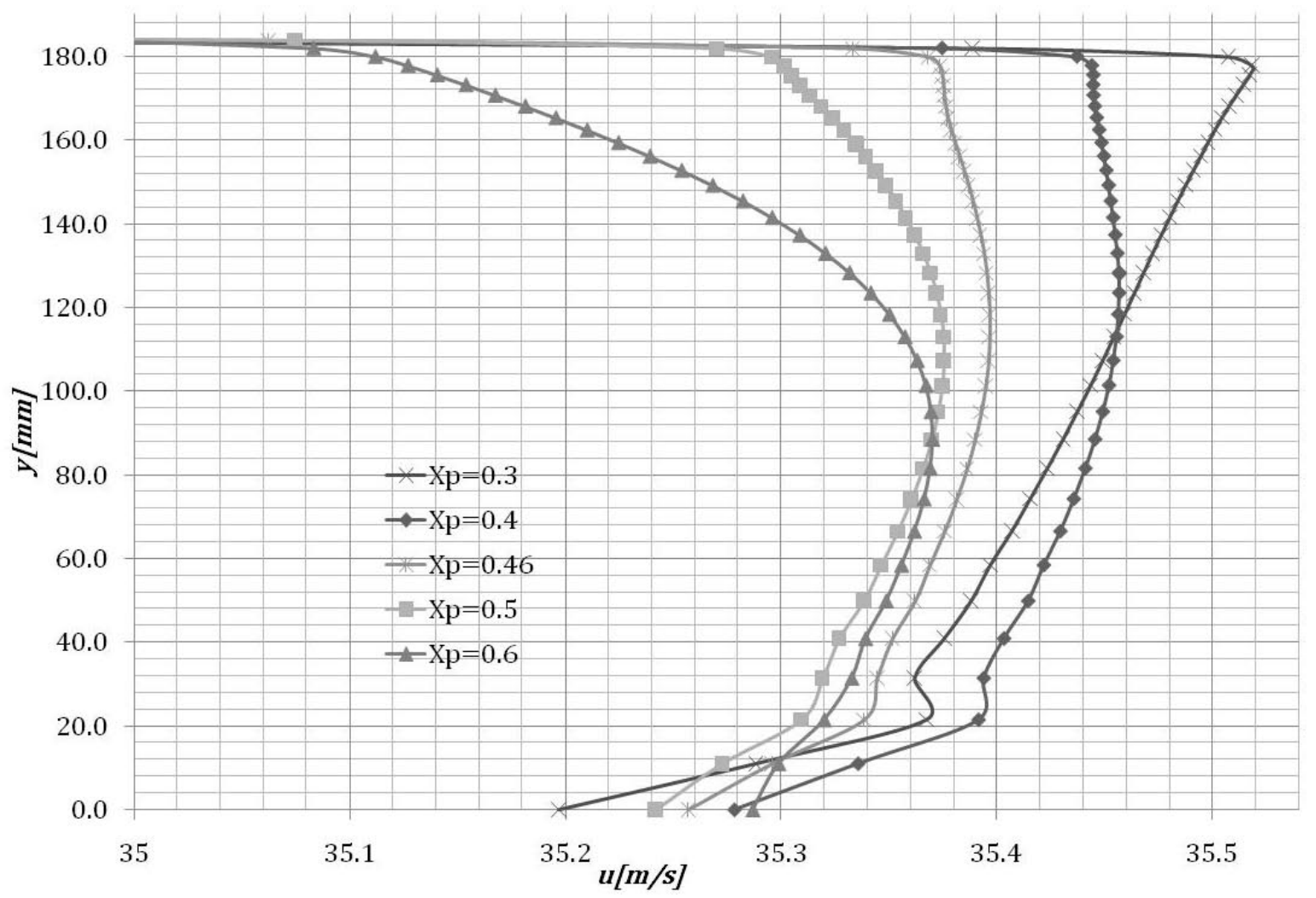

Figure AC5. Velocity profiles, for several Xp. XY plane, Inlet velocity: $3.9 \mathrm{~m} / \mathrm{s}$

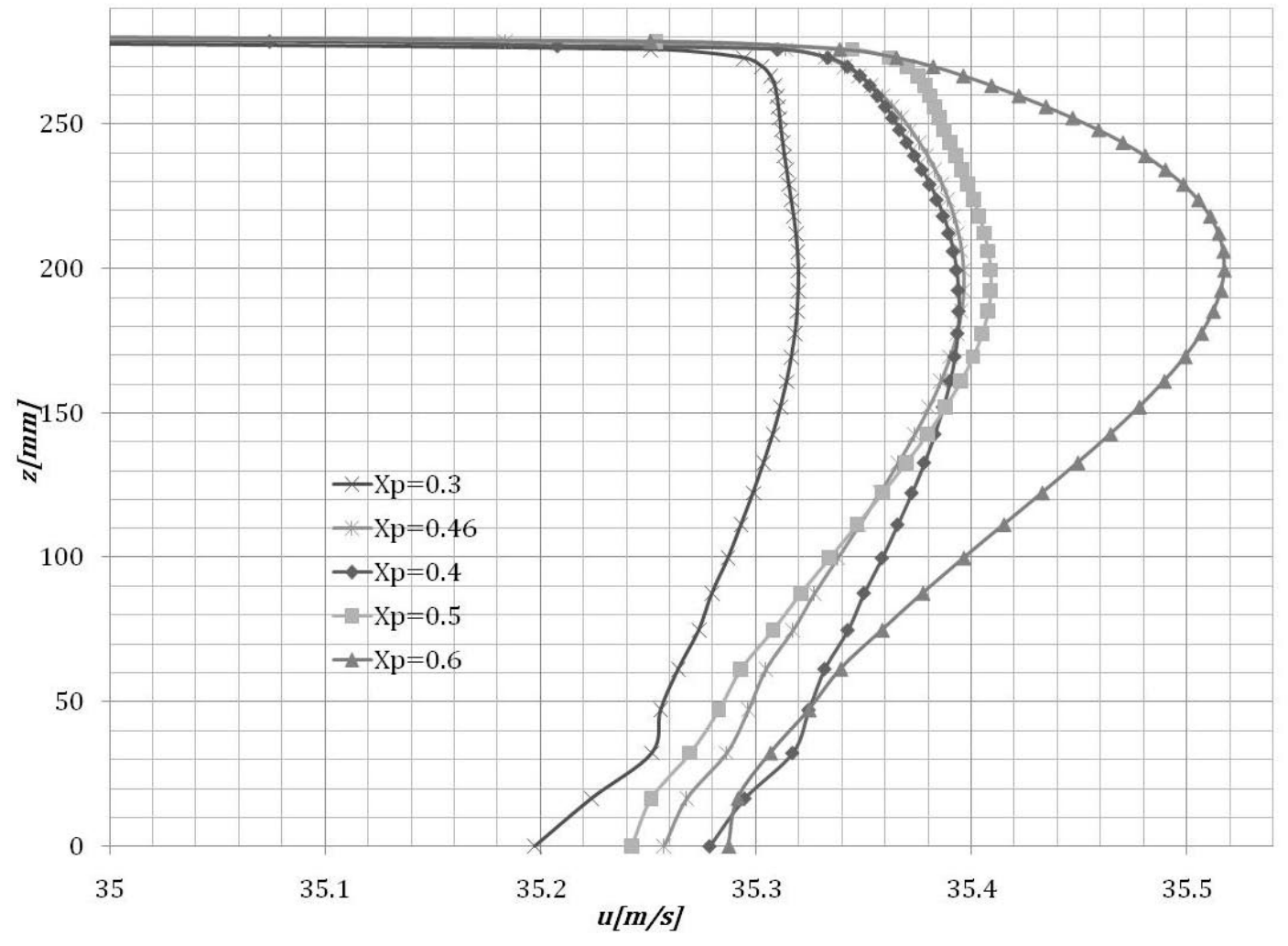

Figure AC6. Velocity profiles, for several Xp. XZ plane, Inlet velocity: $3.9 \mathrm{~m} / \mathrm{s}$ 


\section{APPENDIX D}

\section{PRESSURE DISTRIBUTION IN THE CONTRACTION}




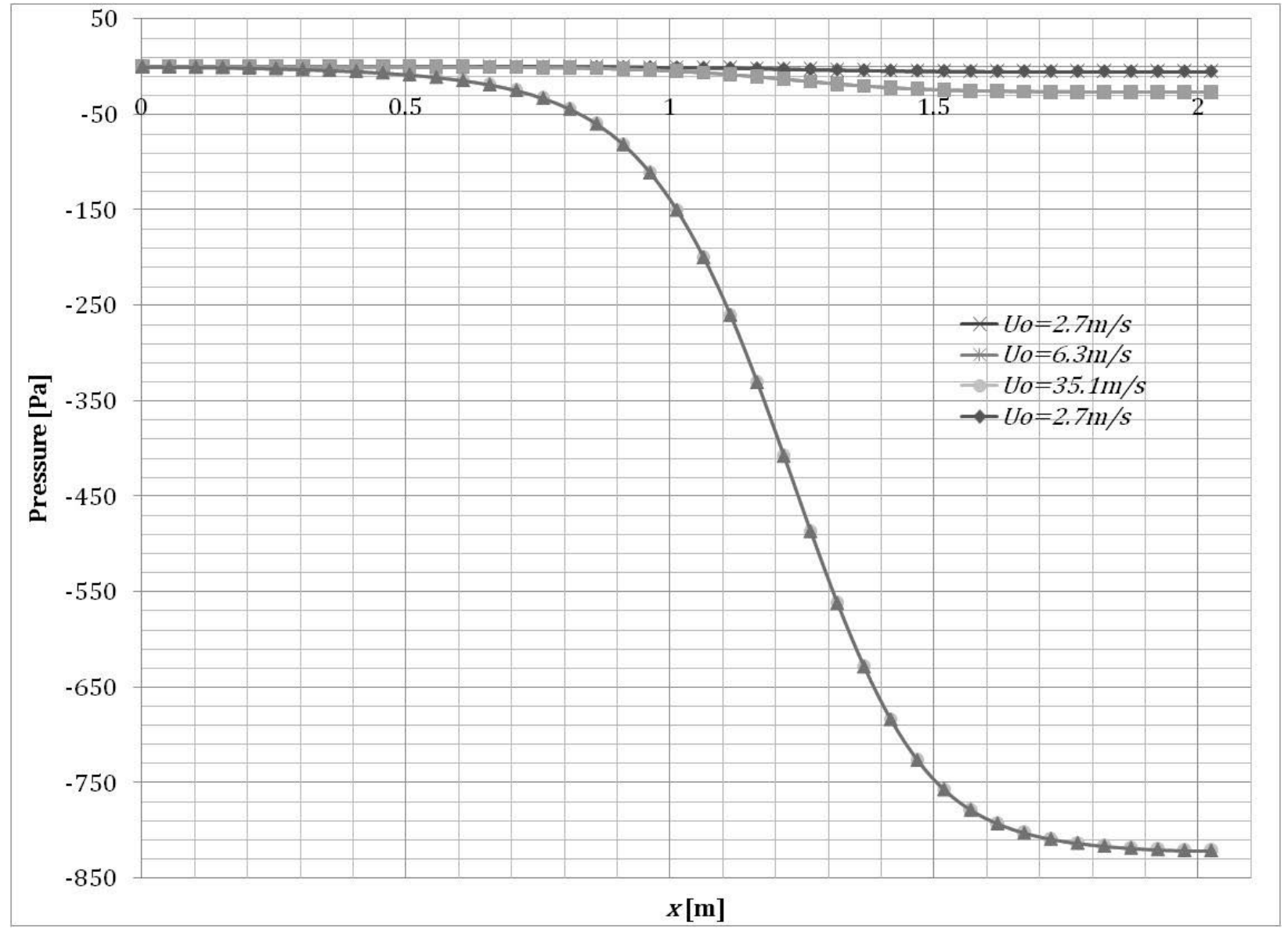

Figure AD1. Pressure distribution in the contraction for several test section velocities. The contraction inlet is located at $x=0$. Differential pressure is shown, the reference pressure is the inlet pressure 


\section{APPENDIX E}

CONTRACTION WALL SHAPE COORDINATES 


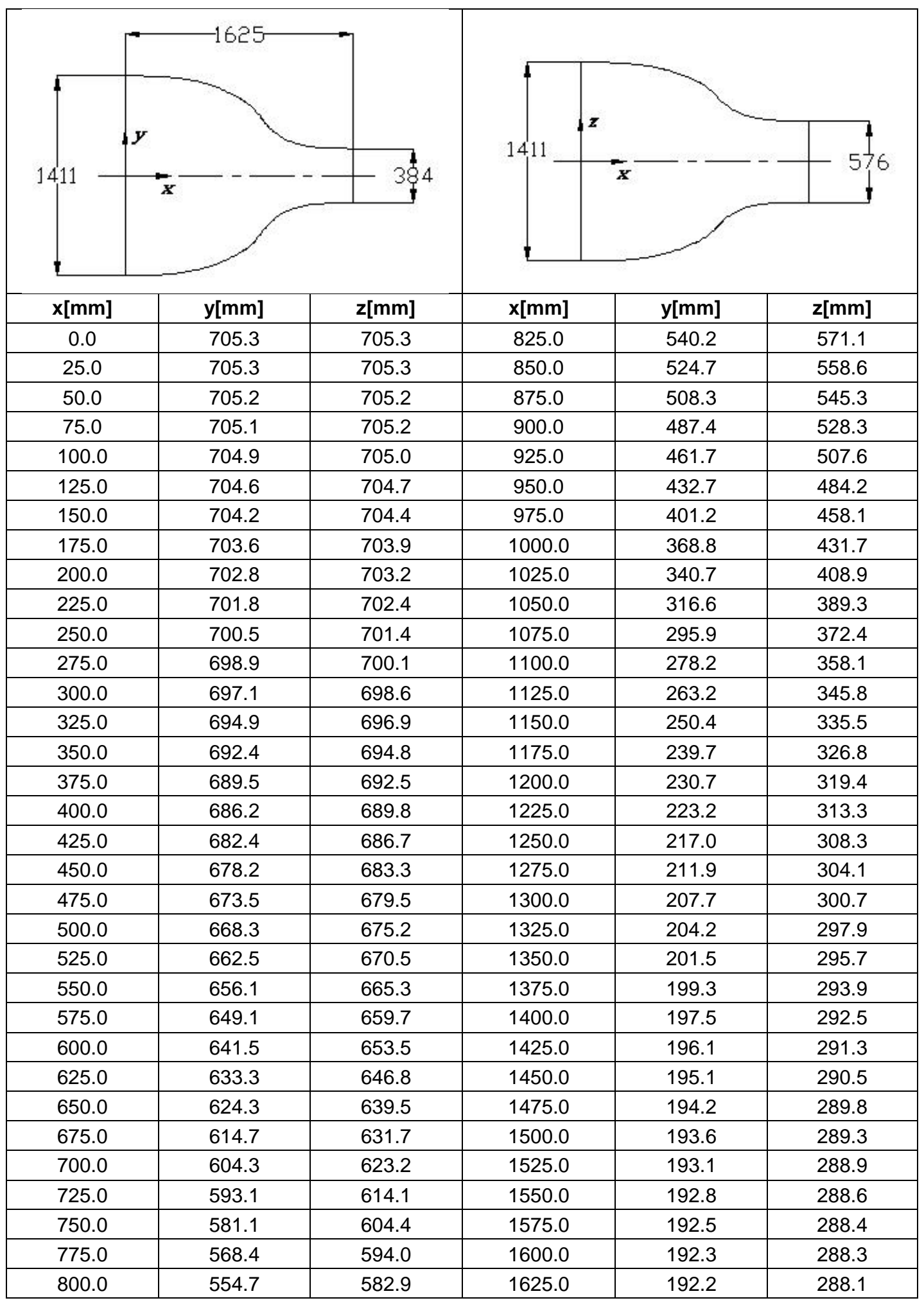

